\title{
Multifrequency Astrophysics (A pillar of an interdisciplinary approach for the knowledge of the physics of our Universe)
}

\author{
Franco Giovannelli** \\ INAF - Istituto di Astrofisica e Planetologia Spaziali, Via del Fosso del Cavaliere, 100, 00133 \\ Roma, Italy \\ E-mail: Eranco.giovannellieiaps.inaf.it
}

\section{Lola Sabau-Graziati}

INTA-Dpt. Cargas Utiles y Ciencias del Espacio, C/ra de Ajalvir, Km 4 - E28850 Torrejón de Ardoz, Madrid, Spain

E-mail: sabaumdeinta.es

We will discuss the importance of the "Multifrequency Astrophysics" as a pillar of an interdisciplinary approach for the knowledge of the physics of our Universe. Indeed, as largely demonstrated in the last decades, only with the multifrequency observations of cosmic sources it is possible to get near the whole behaviour of a source and then to approach the physics governing the phenomena that originate such a behaviour. In spite of this, a multidisciplinary approach in the study of each kind of phenomenon occurring in each kind of cosmic source is even more powerful than a simple "astrophysical approach". A clear example of a multidisciplinary approach is that of "The Bridge between the Big Bang and Biology". This bridge can be described by using the competences of astrophysicists, planetary physicists, atmospheric physicists, geophysicists, volcanologists, biophysicists, biochemists, and astrobiophysicists. The unification of such competences can provide the intellectual framework that will better enable an understanding of the physics governing the formation and structure of cosmic objects, apparently uncorrelated with one another, that on the contrary constitute the steps necessary for life (e.g. Giovannelli, 2001). Indeed, a lot of the future research in astrophysics will be focussed on the discovery of exoplanets and on the possibility to detect signals for alien life somewhere in the Galaxy. An extension to a multidisciplinary approach is coming from the use of historical news reported in "old chronicle" that are a fundamental source for the newborn "archaeoastronomy". In this paper we will provide several example that marked the continuous evolution on the knowledge of the physics of our Universe.

This paper is a summary of an updated version of the book "The Impact of the Space Experiments on Our Knowledge of the Physics of the Universe" published in 2004 by the Kluwer Academic Publishers, reprinted from the review paper by Giovannelli, F. \& Sabau-Graziati, L.: 2004, Space Sci. Rev. 112, 1-443 (GSG2004), and subsequent considered lucubrations.

XII Multifrequency Behaviour of High Energy Cosmic Sources Workshop

12-17 June, 2017

Palermo, Italy

\footnotetext{
* Speaker.

${ }^{\dagger}$ A footnote may follow.
} 


\section{Introduction}

The Bridge between the Big Bang and Biology undoubtedly exists, as discussed in the book edited by Giovannelli (2001). The big problem is how to cross this bridge, and the main question is: what are the experimental tools for understanding the pillars of this Bridge?

In order to cross this bridge, as always when we cross a bridge, we MUST advance slowly, step by step, with continuity, because everything is smoothly linked in the "magma" of the Universe, from the infinitely small to infinitely big, as sketched in Fig. 1 (Rees, 1988) "Origins. The Darwin College lectures (1986)" (Fabian, A.C., 1988).

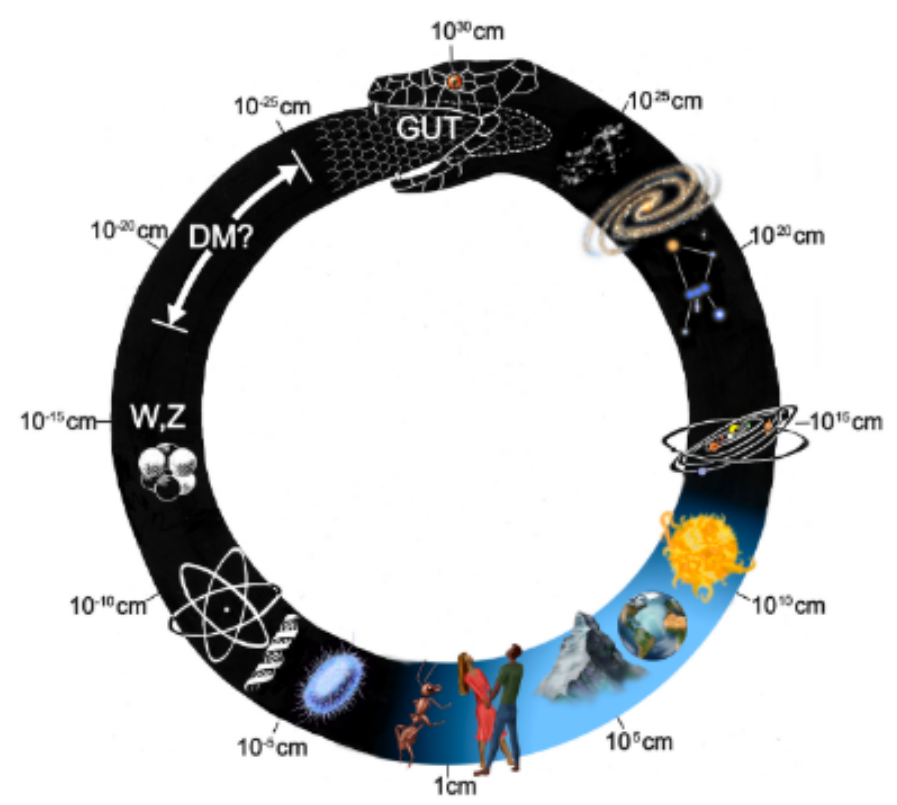

Figure 1: From the infinitely small to infinitely big (adopted by Rees, 1988).

Indeed, if we look at the Fig. 2 (left panel) - where a section of the metabolic network of a "simple" bacterium is shown - we can note that each point (each chemical compound) is connected to any other point through the complexity of the network (Luisi \& Capra, 2014) exactly the same occurring in the "cosmic network" where each point is connected to any other point through the complexity of the network as shown in Fig. 2 (right panel) (https://it.wikipedia.org/wiki/Cosmologia del plasma). The large-scale structure of the Universe, as traced by the distribution of galaxies, is now being revealed by large-volume cosmological surveys. The structure is characterized by galaxies distributed along filaments, the filaments connecting in turn to form a percolating network. Shandarin, Habib \& Heitmann (2010) objective was to quantitatively specify the underlying mechanisms that drive the formation of the cosmic network. By combining percolation-based analyses with N-body simulations of gravitational structure formation, they elucidate how the network has its origin in the properties of the initial density field (nature) and how its contrast is then amplified by the nonlinear mapping induced by the gravitational instability (nurture).

We can strain our eyes into the distance considering that all the people shown in Fig. 3 belong to the humanity. They follow the cycle: birth, growth, aging death, which is exactly the same cycle 

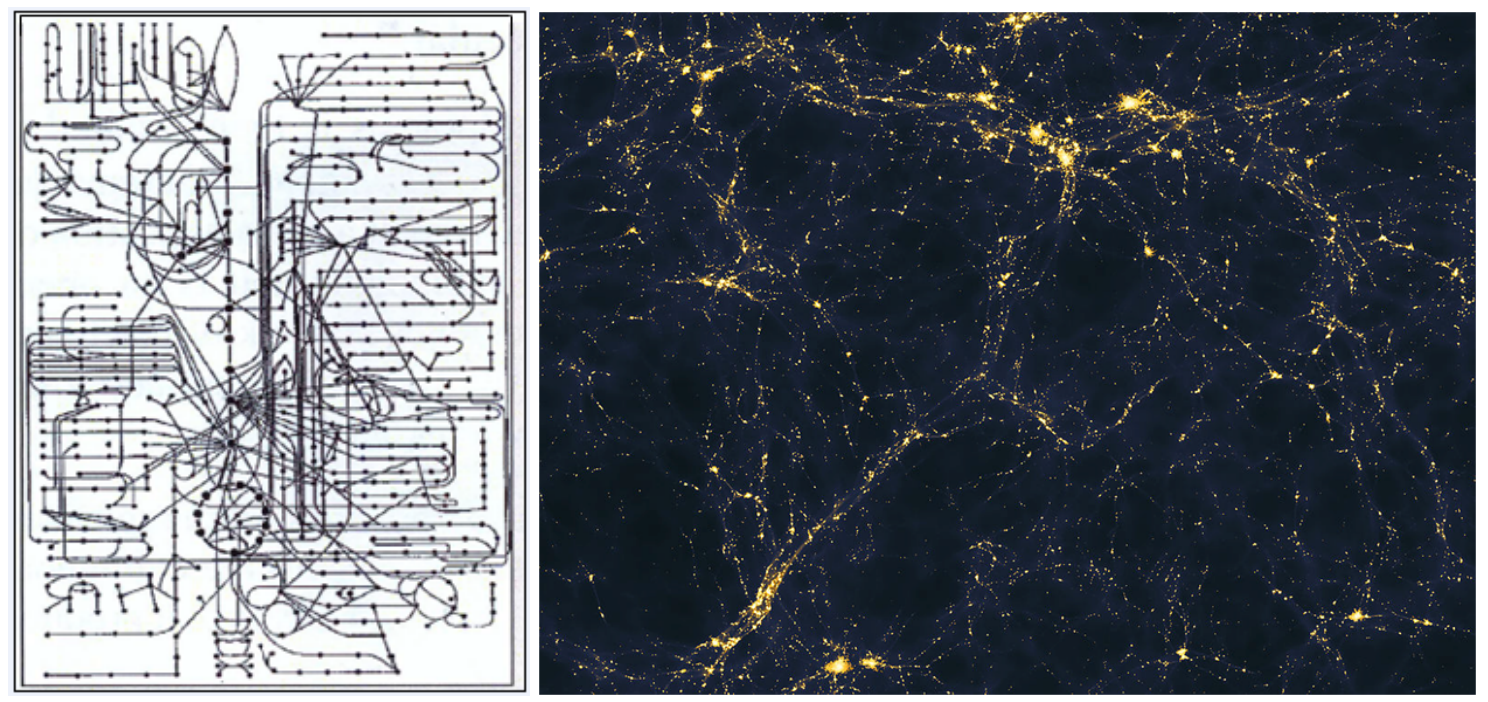

Figure 2: Left panel: Section of the metabolic network of a "simple" bacterium (Luisi \& Capra, 2014). Right panel: the "cosmic network" (https://it.wikipedia.org/wiki/Cosmologia del plasma).

experienced by all the components of the Universe. Therefore for a complete understanding of the history of the Universe it is necessary to search along that cycle.
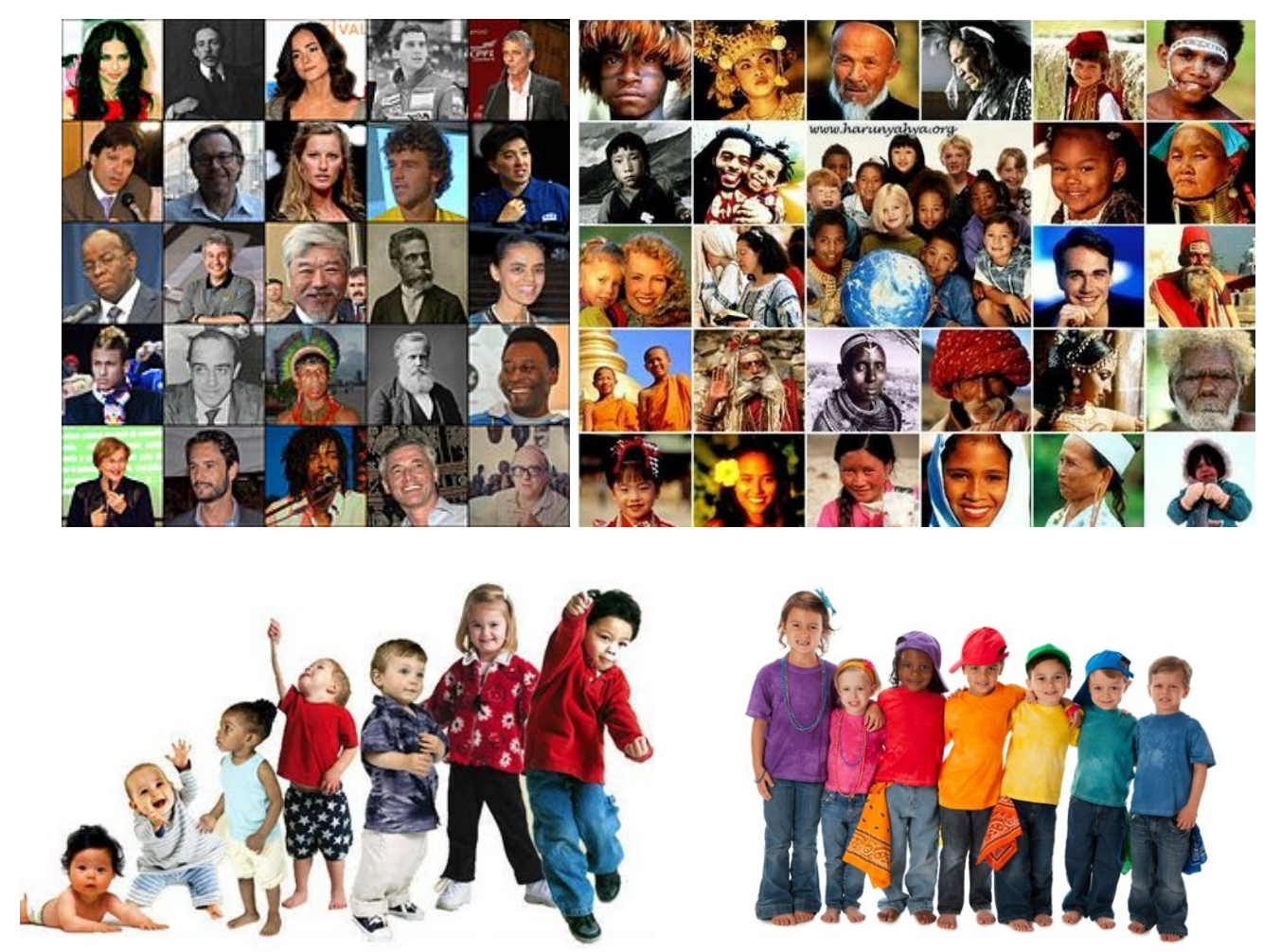

Figure 3: From left upper panel clockwise: a) https://commons.wikimedia.org/wiki/File:Brazilians.jpg; b) 1456962-419386468183546-1930497595-n.jpg; c) fotonoticia-20150407101323-645.jpg; d) desarrollo psicosexual freud citlali.jpg. 
As Albert Einstein affirmed, we can't solve problems by using the same kind of thinking we used when we created them. We can add something more, by using the wisdom: we can attach each kind of problem in a way as general as possible, and in any case it is necessary to go on without blinkers.

A fundamental question naturally arises: what is now the situation about our knowledge of the Universe? The answer is discouraging: we know a very small part of it, and not very well.

Indeed, Fig. 4 shows schematically such a situation. We can really discuss only on $\sim 5 \%$ of the content of the Universe. The remnant of $\sim 95 \%$ is almost completely unknown. However, the recent fundamental progress in gravitational astronomy could open an incredible source of information about this "unknown" content.

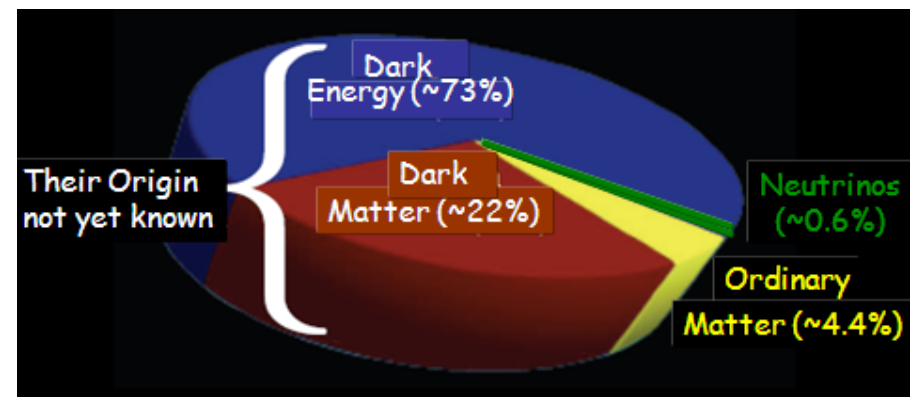

Figure 4: The cosmic budget.

\section{The tools for exploring the Universe}

The Universe manifests not only through electromagnetic radiation but also through astroparticles, including neutrinos, and recently through gravitational waves. Therefore, multifrequency observations, possibly simultaneous, are fundamental in photonic astrophysics, particle astrophysics, and gravitational wave astrophysics.

Thus it is evident the necessity of many kinds of experiments in different frequency regions. Upstream of this we have to distinguish two great classes of experiments: big experiments and small experiments.

\subsection{Big experiments}

Several examples of big experiments are:

- the Astrometry Mission GAIA (Rix \& Bovy, 2013) is making the largest, most precise threedimensional map of our Galaxy by surveying more than a thousand million stars. GAIA will monitor each of its target stars about 70 times over a five-year period. It will precisely chart their positions, distances, movements, and changes in brightness. It is expected to discover hundreds of thousands of new celestial objects, such as extra-solar planets and brown dwarfs, and observe hundreds of thousands of asteroids within our own Solar System. The mission will also study about 500,000 distant quasars and will provide stringent new tests of Albert Einstein's General Theory of Relativity. 
GAIA is providing strong impact on stellar evolution and in calibrating the energetic of cosmic sources.

- The European Extremely Large Telescope (E-ELT) is a revolutionary scientific project for a $40 \mathrm{~m}$-class telescope that will allow us to address many of the most pressing unsolved questions about our Universe.

The E-ELT will be the largest optical/near-infrared telescope in the world and will gather 13 times more light than the largest optical telescopes existing today. The E-ELT will be able to correct for the atmospheric distortions (i.e., fully adaptive and diffraction-limited) from the start, providing images 16 times sharper than those from the Hubble Space Telescope. The E-ELT will vastly advance astrophysical knowledge by enabling detailed studies of planets around other stars, the first galaxies in the Universe, super-massive black holes, and the nature of the Universe's dark sector (Gilmozzi \& Spyromilio, 2007).

The final approval of E-ELT occurred at ESO on December 3, 2014 (de Zeeuw, Tamai \& Liske, 2014). On May 25, 2016 ESO Signs Largest Ever Ground-based Astronomy Contract for ELT Dome and Telescope Structure (eso1617 - Organisation Release). An expanded view of the Universe (Science with the European Extremely Large Telescope) can be found in http://www.eso.org/sci/facilities/eelt/docs/.

Figure 5 shows the comparison of E-ELT with the pyramids of Egypt (Gilmozzi, 2013).

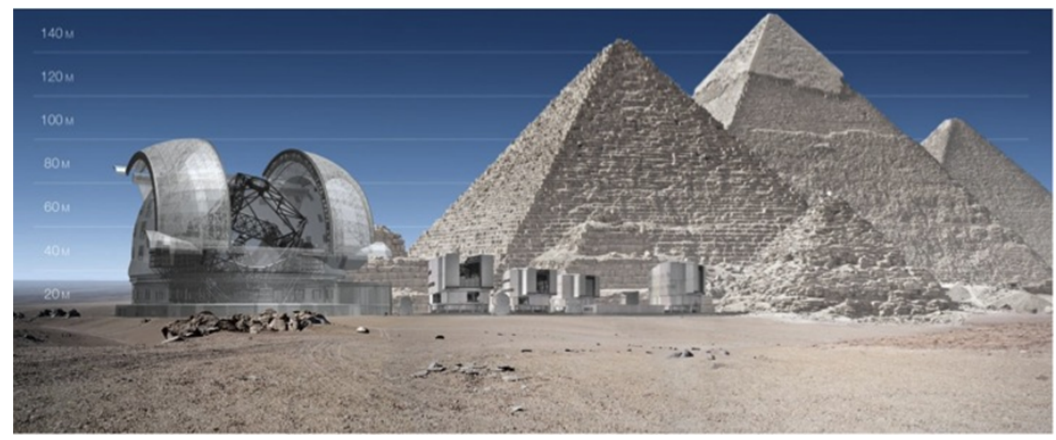

Figure 5: E-ELT versus Egyptian pyramids (adopted from Gilmozzi, 2013).

- The James Webb Space Telescope (JWST) will be a giant leap forward in our quest to understand the Universe and our origins. JWST will examine every phase of cosmic history: from the first luminous glows after the Big Bang to the formation of galaxies, stars, and planets to the evolution of our own solar system.

Figure 6 sketches the different phases of the cosmic history (upper panel) and the structure of the JWST (lower panel) (https://jwst.nasa.gov/science.html).

- The THESEUS mission is designed to vastly increase the discovery space of the high energy transient phenomena over the entirety of cosmic history (http://www.isdc.unige.ch/theseus/). The main scientific goals of the proposed mission are to:

a. Explore the Early Universe (cosmic dawn and reionization era) by unveiling a complete census of the Gamma-Ray Burst (GRB) population in the first billion years. 


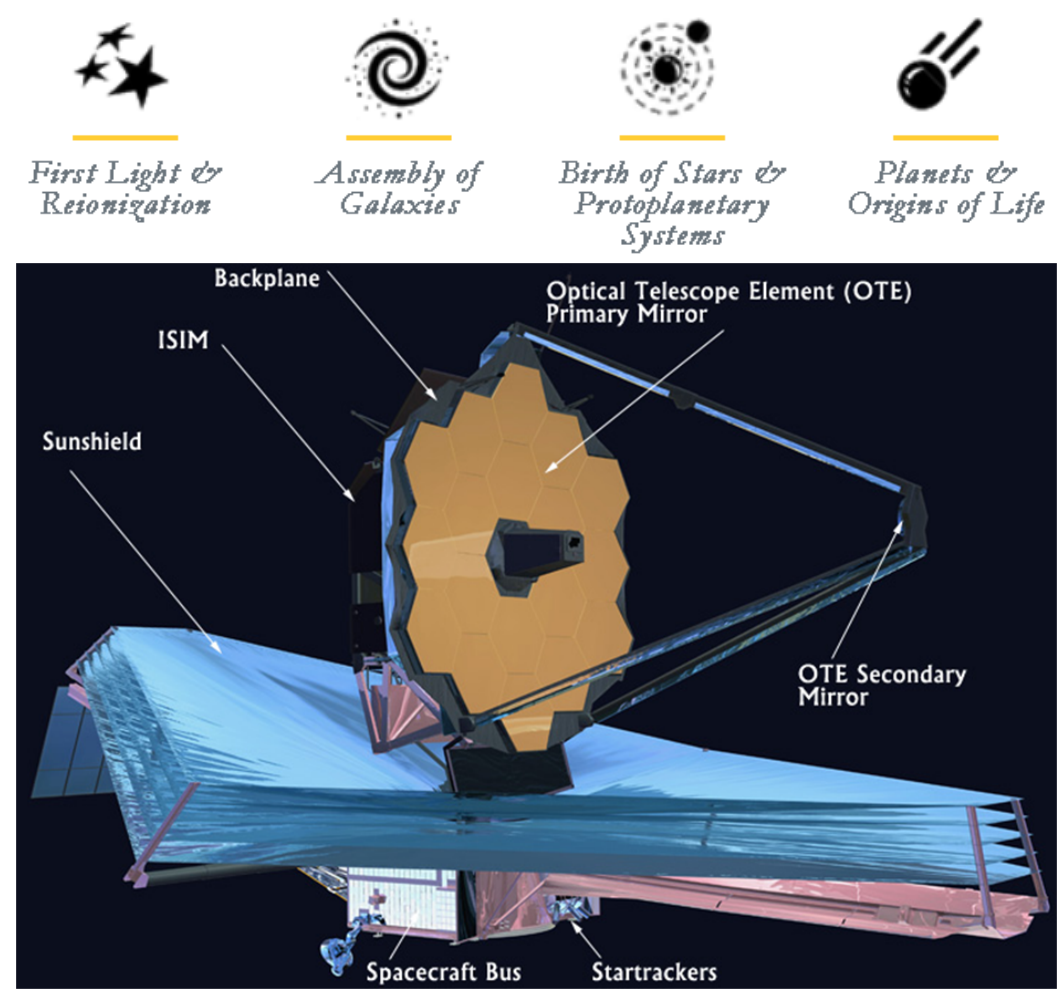

Figure 6: (upper panel: the different phases of the cosmic history; lower panel: the structure of the JWST (https://jwst.nasa.gov/science.html).

b. Perform an unprecedented deep monitoring of the X-ray transient Universe.
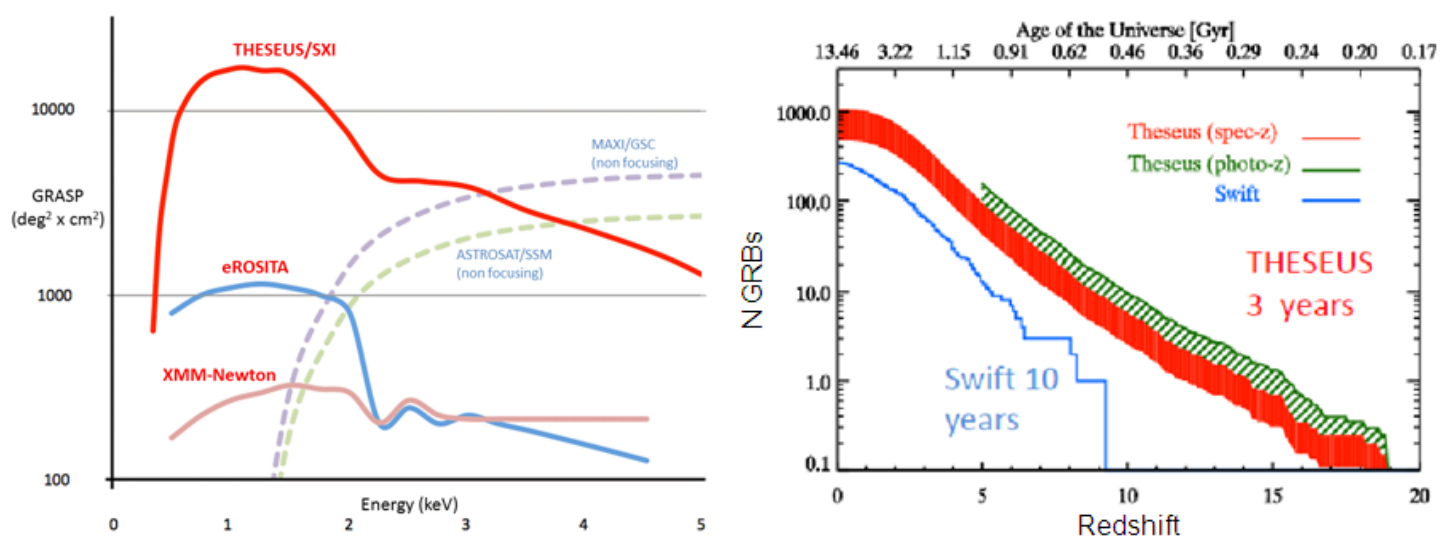

Figure 7: (Left panel: the GRASP (FoV versus Effective Area) as function of energy; right panel: the cumulative distribution of GRBs with redshift determination as a function of the redshift for Swift (in $10 \mathrm{yr}$ ) and the prediction for THESEUS (in $3 \mathrm{yr}$ ) (http://www.isdc.unige.ch/theseus/).

Figure 7 shows the GRASP FoV versus Effective Area) as function of energy (left panel) and the cumulative distribution of GRBs with redshift determination as a function of the redshift for Swift (in $10 \mathrm{yr}$ ) and the prediction for THESEUS (in $3 \mathrm{yr}$ ).

- e-ASTROGAM ("enhanced ASTROGAM") is a breakthrough Observatory space mission, 
with a detector composed by a Silicon tracker, a calorimeter, and an anticoincidence system, dedicated to the study of the non-thermal Universe in the photon energy range from $0.3 \mathrm{MeV}$ to $3 \mathrm{GeV}$ - the lower energy limit can be pushed to energies as low as $150 \mathrm{keV}$, albeit with rapidly degrading angular resolution, for the tracker, and to $30 \mathrm{keV}$ for calorimetric detection. The mission is based on an advanced space-proven detector technology, with unprecedented sensitivity, angular and energy resolution, combined with polarimetric capability. Thanks to its performance in the $\mathrm{MeV}-\mathrm{GeV}$ domain, substantially improving its predecessors, eASTROGAM will open a new window on the non-thermal Universe, making pioneering observations of the most powerful Galactic and extragalactic sources, elucidating the nature of their relativistic outflows and their effects on the surroundings. With a line sensitivity in the $\mathrm{MeV}$ energy range one to two orders of magnitude better than previous generation instruments, e-ASTROGAM will determine the origin of key isotopes fundamental for the understanding of supernova explosion and the chemical evolution of our Galaxy. The mission will provide unique data of significant interest to a broad astronomical community, complementary to powerful observatories such as LIGO-Virgo-GEO600-KAGRA, SKA, ALMA, E-ELT, TMT, LSST, JWST, Athena, CTA, IceCube, KM3NeT, and the promise of eLISA (De Angelis, et al., 2017).
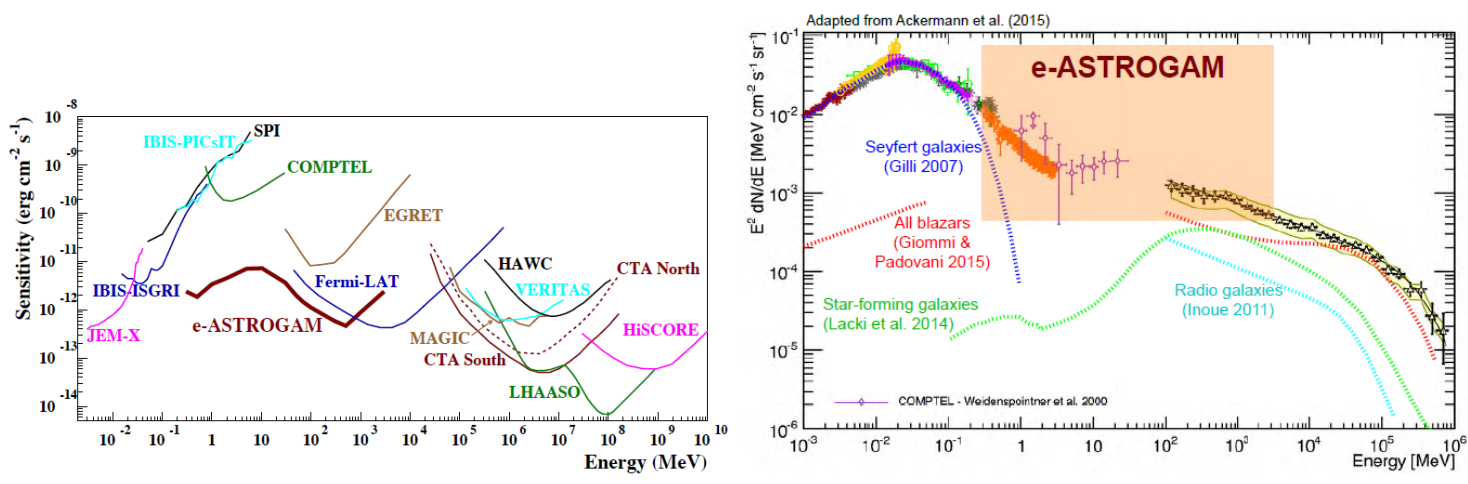

Figure 8: (Left panel: the sensitivity of e-ASTROGAM compared with those of the past, present and future experiments; right panel: the compilation of measurements of extragalactic sources between $1 \mathrm{keV}$ and 820 $\mathrm{GeV}$, The semitransparent band indicates the energy region in which e-ASTROGAM will strongly improve on present knowledge (De Angelis, et al., 2017).

Figure 8 shows: in the left panel the sensitivity of e-ASTROGAM compared with those of the past, present and future experiments; in the right panel the compilation of measurements of extragalactic sources between $1 \mathrm{keV}$ and $820 \mathrm{GeV}$, The semitransparent band indicates the energy region in which e-ASTROGAM will strongly improve on present knowledge (De Angelis, et al., 2017).

- Cosmic Ray experiments: Figure 9 shows the various experiments that will cover different bands of the cosmic ray spectrum. Some of them are already working, some of them will operate in the future (Spillantini, 2008, 2009).

- The GAMMA-400 gamma-ray telescope: the next absolutely necessary step in the development of extraterrestrial high-energy $\gamma$-ray astronomy is the improvement of the physical and 


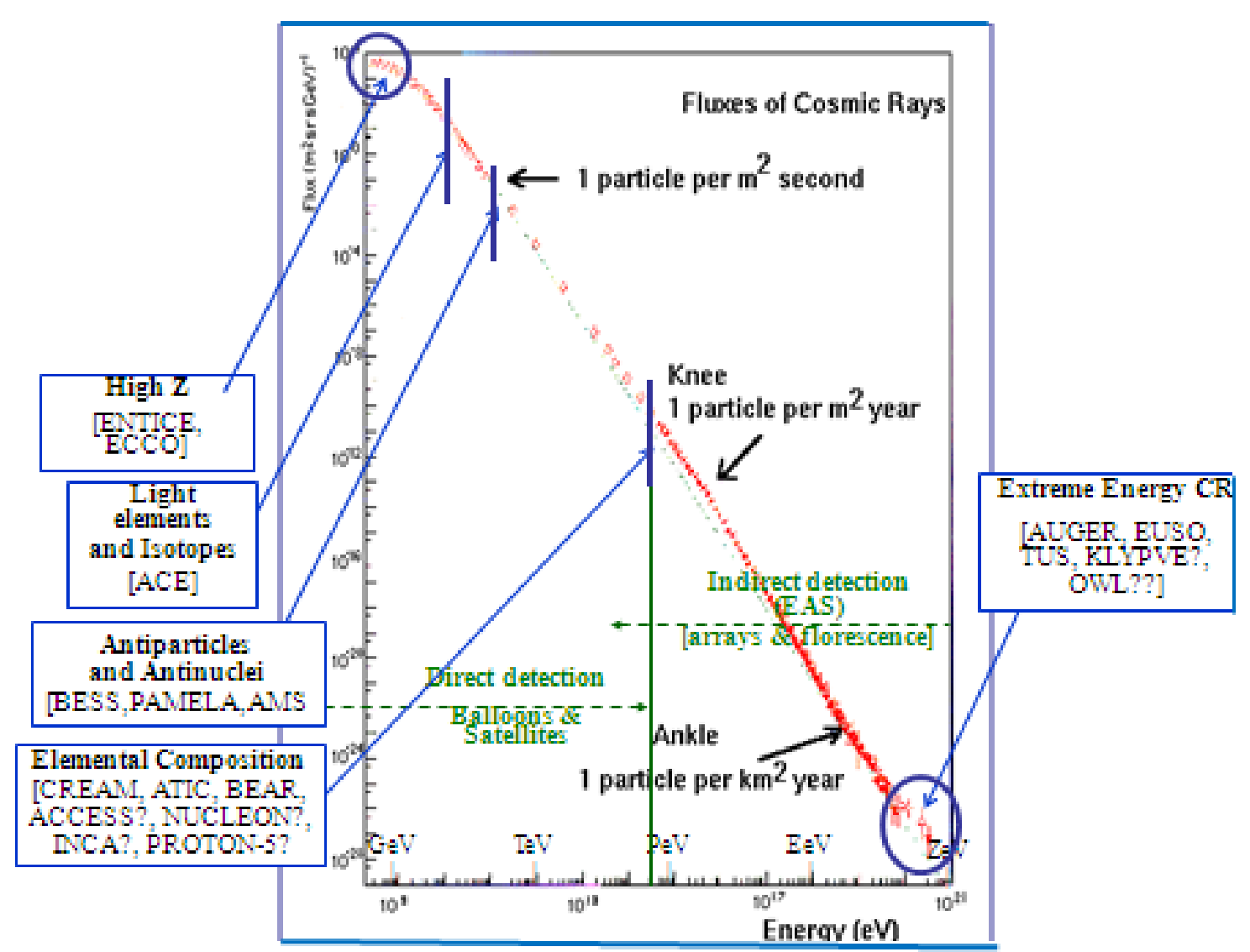

Figure 9: The many experiments that are exploring and will explore differenht bands of the cosmic ray spectrum (Spillantini, 2008).

technical characteristics of $\gamma$-ray telescopes, especially the angular and energy resolutions. Such a new generation telescope will be GAMMA-400, which will be installed onboard the Russian space observatory.

The GAMMA-400 gamma-ray telescope is intended to measure the fluxes of gamma-rays and cosmic-ray electrons and positrons in the energy range from $100 \mathrm{MeV}$ to several $\mathrm{TeV}$. Such measurements concern the following scientific tasks: investigation of point sources of gamma-rays, studies of the energy spectra of Galactic and extragalactic diffuse emission, studies of gamma-ray bursts and gamma-ray emission from the Sun, as well as high precision measurements of spectra of high-energy electrons and positrons. Also the GAMMA- 400 instrument provides the possibility for protons and nuclei measurements up to knee. But the main goal for the GAMMA-400 mission is to perform a sensitive search for signatures of dark matter particles in high-energy gamma-ray emission (Topchiev et al., 2016a,b, and the references therein).

The GAMMA-400 will operate in the highly elliptic orbit continuously for a long time with the unprecedented angular $\left(\sim 0.01^{\circ}\right.$ at $\left.\mathrm{E}_{\gamma}=100 \mathrm{GeV}\right)$ and energy $\left(\sim 1 \%\right.$ at $\left.\mathrm{E}_{\gamma}=100 \mathrm{GeV}\right)$ resolutions better than the Fermi-LAT, as well as ground $\gamma$-ray telescopes, by a factor of 5- 
10. GAMMA-400 will permit to resolve $\gamma$-rays from annihilation or decay of dark matter particles, identify many discrete sources (many of which are variable), to clarify the structure of extended sources, to specify the data on the diffuse emission (Topchiev et al., 2017).

- The Large Hadron Collider (LHC) is the world's largest and most powerful particle accelerator. It first started up on 10 September 2008, and remains the latest addition to CERN's accelerator complex. The LHC consists of a $27 \mathrm{~km}$ ring of superconducting magnets with a number of accelerating structures to boost the energy of the particles along the way. Figure 10 shows a partial view of the tunnel hosting the accelerator.

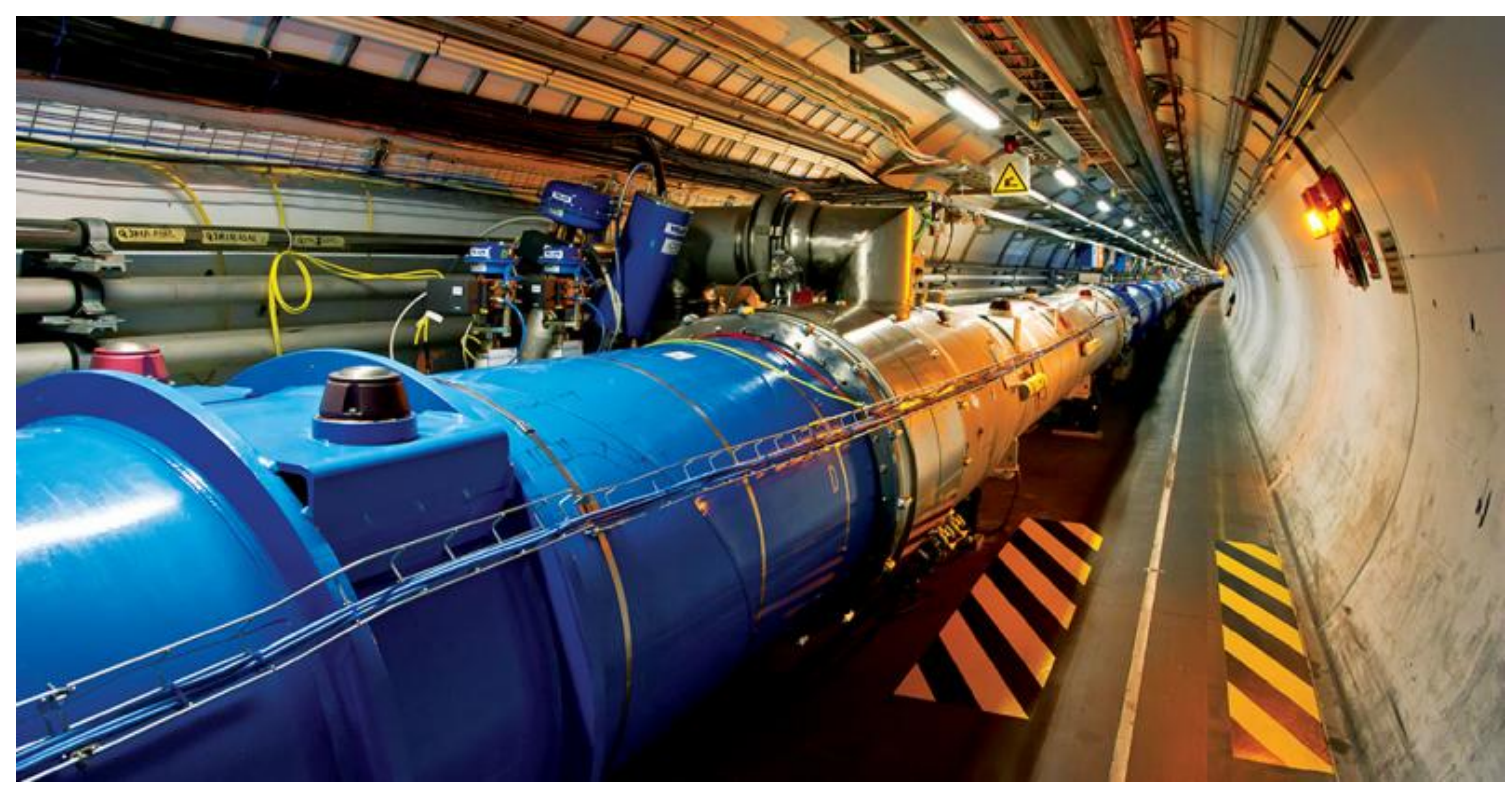

Figure 10: The Large Hadron Collider is the world's largest and most powerful particle accelerator (Image: CERN).

All the controls for the accelerator, its services and technical infrastructure are housed under one roof at the CERN Control Centre. From here, the beams inside the LHC are made to collide at four locations around the accelerator ring, corresponding to the positions of four particle detectors - ATLAS (A Toroidal LHC ApparatuS), CMS (Compact Muon Solenoid), ALICE (A large Ion Collider Experiment) and LHCb (Large Hadron Collider beauty). There are three more smaller experiments: LHCf (Large Hadron Collider forward), TOTEM (TOTal Elastic and diffractive cross section Measurement), and MoEDAL (Monopole and Exotics Detector at the LHC). TOTEM is installed close to the CMS interaction point, LHCf is installed near ATLAS, and MoEDAL is close to the LHCb detector. The position of the four main experiments are shown in Fig. 11 (https://home.cern/topics/large-hadron-collider).

The LHC will answer some of the fundamental open questions in physics, concerning the basic laws governing the interactions and forces among the elementary objects, the deep structure of space and time, and in particular the interrelation between quantum mechanics and general relativity. Data is also needed from high-energy particle experiments to suggest which versions of current scientific models are more likely to be correct - in particular to 


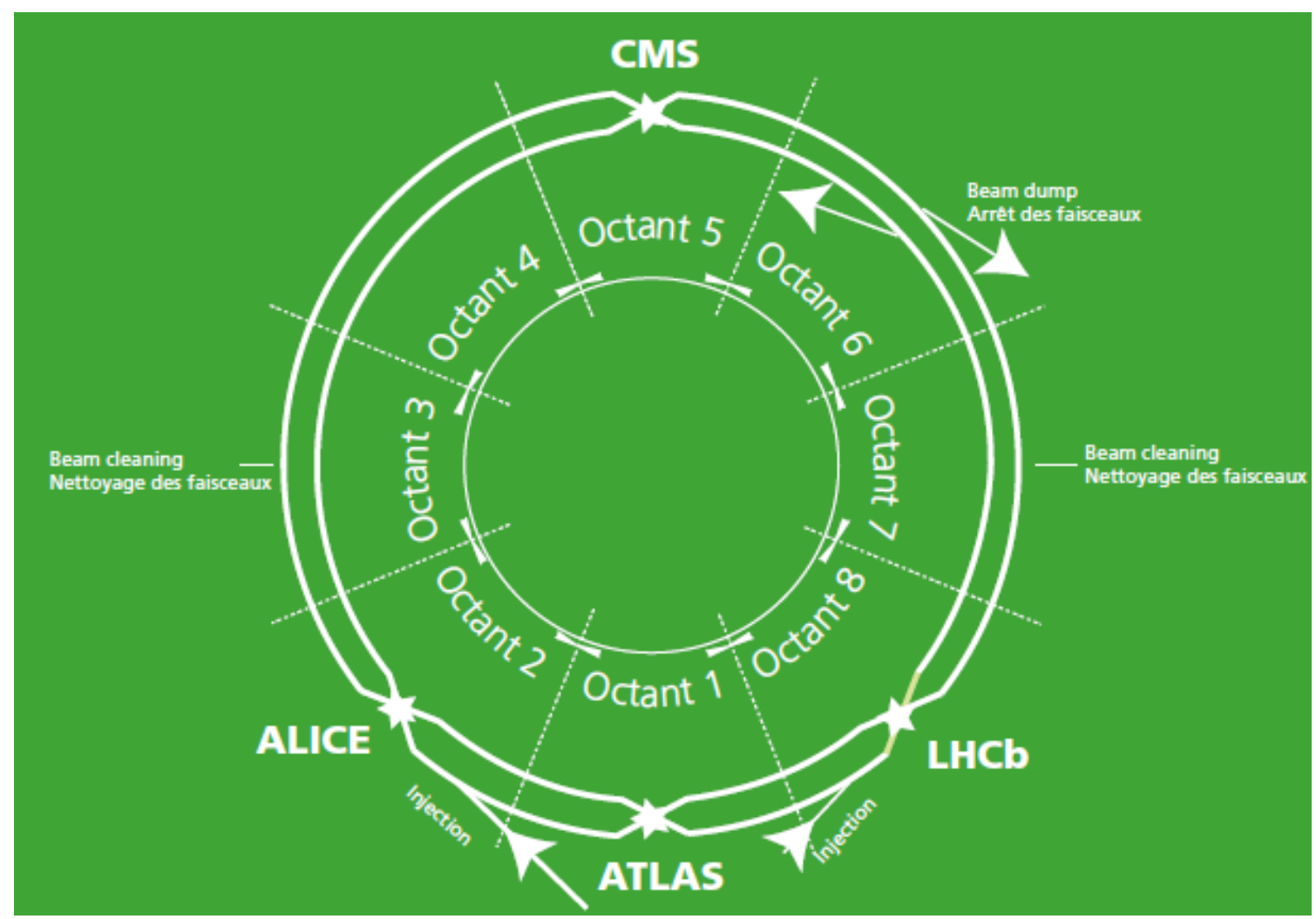

Figure 11: Localization of the four main experiments at LHC (CERN-Brossure-2017-002-Eng, p. 26).

choose between the Standard Model and Higgs-less model and to validate their predictions and allow further theoretical development. Many theorists expect new physics beyond the Standard Model to emerge at the TeV energy level, as the Standard Model appears to be unsatisfactory. Issues explored by LHC collisions include i) the mass of elementary particles being generated by the Higgs mechanism; ii) supersymmetry, an extension of the Standard Model and Poincaré symmetry; iii) extra dimensions, as predicted by various models based on string theory; iv) the nature of the dark matter that appears to account for $\sim 27 \%$ of the mass-energy of the universe; $v$ ) answer to the question if the electroweak force and the strong nuclear force are just different manifestations of one universal unified force, as predicted by various Grand Unification Theories; vi) why the fourth fundamental force (gravity) is so many orders of magnitude weaker than the other three fundamental forces; vii) are there additional sources of quark flavour mixing, beyond those already present within the Standard Model?; viii) why are there apparent violations of the symmetry between matter and antimatter?; ix) what are the nature and properties of quark-gluon plasma, thought to have existed in the early universe and in certain compact and strange astronomical objects today?

Some important results obtained with the LHC will be discussed later.

- The BICEP (Background Imaging of Cosmic Extragalactic Polarization) and the Keck Array are a series of cosmic microwave background (CMB) experiments. They aim to measure the polarization of the $\mathrm{CMB}$; in particular, measuring the $\mathrm{B}$-mode of the $\mathrm{CMB}$. The experiments 
have had four generations of instrumentation, consisting of BICEP1, BICEP2, the Keck Array, and BICEP3. These experiments are observing from the South Pole, and their aims are to discover signatures of Inflation by actually detecting the Cosmic Gravitational Background (CGB) via its weak imprint as the unique B-mode polarization signature of the CMB, directly probing the Universe at an earlier time than ever before. Each generation represents a large increase in sensitivity to B-mode polarization. BICEP1 observed from 2006-2008 with 98 detectors, BICEP2 began observing in the beginning of 2010 with 512 detectors, and the first three of five Keck Array telescopes began observing in the beginning of 2011, each with 512 detectors. The final two Keck Array receivers were deployed during the summer season of 2012. BICEP3, with a total of 2,560 detectors, has been operational since May 2016.

The main goal of BICEP experiments is to test the validity of the theory of the Inflation (Keating et al., 2003; Ogburn IV et al., 2010).

In particular, if Inflation happened immediately after the Big Bang, it would have produced turbulence in the structure of space-time itself-gravitational waves like the kind LIGO detected recently. While these waves would be too weak for LIGO to see, they would twist the orientation of the light, which is known as polarization.

We will discuss later the results coming from BICEP2/Keck-Array and we will comment on their validity.

Of course the list of big experiments is far to be complete, but it is enough to show to the reader the efforts that the international scientific community are facing both for determining the frontier scientific tests to validate the current theories and for the difficulties in providing sufficient budgets for their realization.

\subsection{Small experiments}

In this short excursion about the tools necessary for an advancement of our knowledge of the physics of the Universe, we cannot omit the extreme importance of small experiments, like those Space-based: small-, mini-, micro-, nano-, and cube-satellites, and those Ground-based: small-telescope, and Robotic-telescopes.

Castro-Tirado (2010a) in his review "Robotic Autonomous Observatories: A Historical Perspective" presented a historical introduction to the field of Robotic Astronomy, discussing the basic definitions, the differing telescope control operating systems, observatory managers, as well as a few current scientific applications in that time.

The number of automatic astronomical facilities worldwide continues to grow, and the level of robotisation, autonomy, and networking is increasing as well. This has a strong impact in many astrophysical fields, like the search for extrasolar planets, the monitoring of variable stars in our Galaxy, the study of active galactic nuclei, the detection and monitoring of supernovae, and the immediate followup of high-energy transients such as gamma-ray bursts (Castro-Tirado, 2008, 2010b).

The number of Robotic Autonomous Observatories (RAOs) has rapidly grown. Figure 12 shows the location of more than 100 RAOs worldwide (Castro Cerón, 2011). They are providing excellent results which should be impossible to obtain with the larger telescopes subject to strict scheduling, and in any case not available for long term runs of observations. 


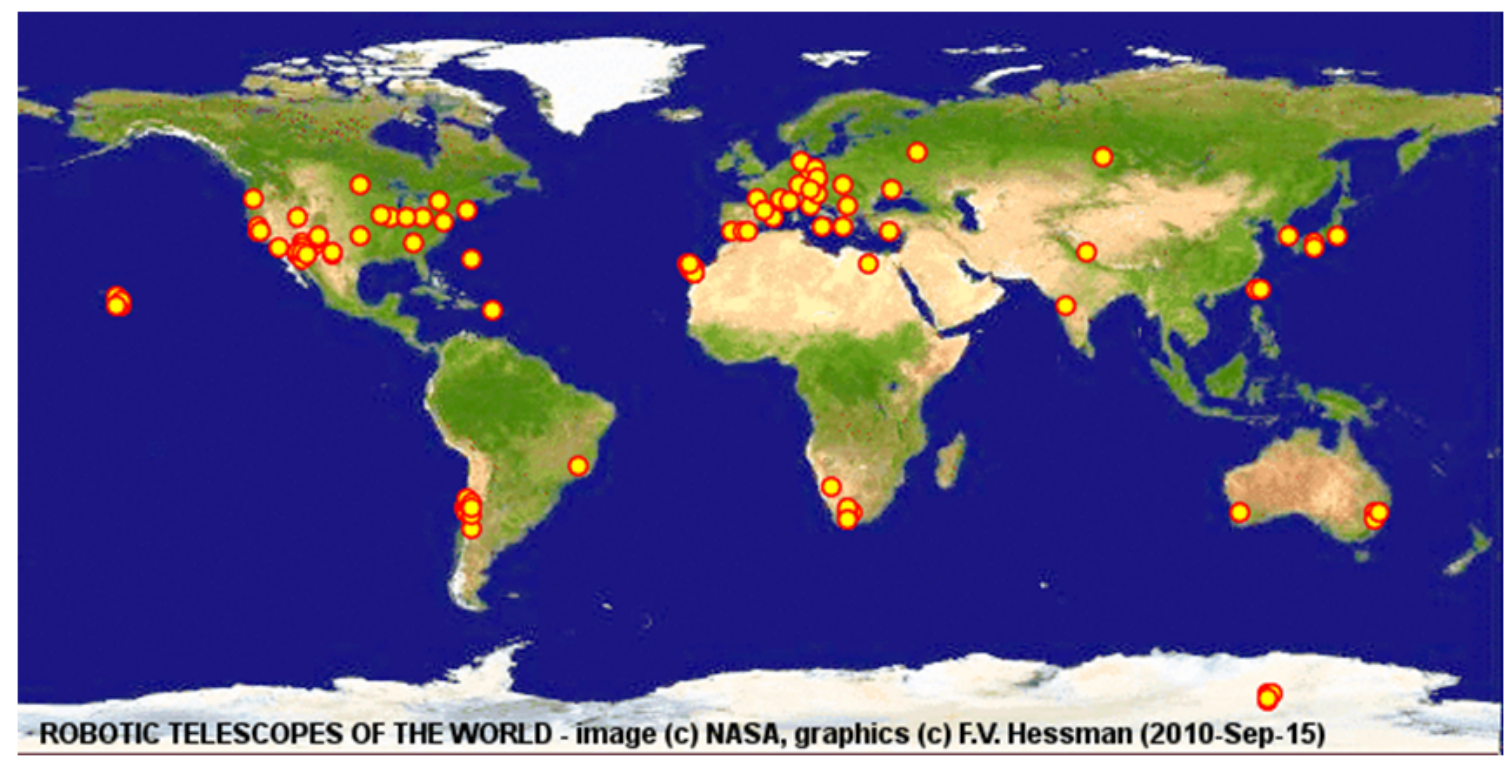

Figure 12: The Robotic Autonomous Observatories worldwide (adopted from Castro Cerón (2011) after Hessman (2010).

The most important news about the many scientific results obtained with the RAOs can be found in the proceedings of the series of Workshops on Robotic Autonomous Observatories (Bloom, Castro-Tirado, Hanlon \& Kotani, 2010; Guziy, Pandey, Tello \& Castro-Tirado, 2012; Tello, Riva, Hiriart \& Castro-Tirado, 2014; Caballero-García, Pandey, Hiriart \& Castro-Tirado, 2016).

Just for giving to the reader a short panorama about the many small ground- and space-based experiments, not necessarily autonomous, we list the follows:

- MITSuME (Multicolor Imaging Telescope for Survey and Monstrous Explosions) has been built to perform Multi-color photometry of NIR/optical afterglow covering the wavebands from $\mathrm{K}_{s}$ to $\mathrm{g}$ ' allowing the photometric redshift measurements up to $\mathrm{z} \sim 10$. Two $50 \mathrm{~cm}$ optical telescopes are built at Akeno, Yamanashi in eastern Japan, and at OAO, Okayama in western Japan. Each telescope has a Tricolor Camera, which allows us to take simultaneous images in $\mathrm{g}^{\prime}, \mathrm{R}_{c}$, and $\mathrm{I}_{c}$ bands. These telescopes respond automatically to GCN alerts and start taking series of tricolor images, which are immediately processed through the analysis pipeline on site. The pipeline consists of source finding, catalog matching, sky coordinates mapping to the image pixels, and photometry of the found sources. An automated search for an optical counterpart is performed. While waiting for GRBs, the MITSuME Telescopes automatically patrol pre-selected interesting objects such as AGNs and galactic transients for multiwavelength studies with Fermi (GLAST) and MAXI (Shimokawabe et al., 2009).

- The CHASE (CHilean Automatic Supernova sEarch) project began in 2007 (Pignata et al., 2009) with the goal to discover young, nearby southern supernovae in order to i) better understand the physics of exploding stars and their progenitors, and ii) refine the methods to derive extra- galactic distances. During the first four years of operation, CHASE has 
produced more than 130 supernovae, being the most successful project of its type in the southern hemisphere (Hamuy et al., 2012).

- The Russian global network of telescopes robot MASTER (Lipunov et al., 2010). MASTER is very fast positioning alert, follow up and survey twin telecopes Global network with own real-time auto-detection software. MASTER goal is One Sky in One Night up to 20-21 mag. The network is spread along the whole world. In the following are reported the MASTER Net Sites:

- MASTER-Amur: Russia, near Blagoveschensk. Blagoveschensk State Pedagogic University.

- MASTER-Tunka: Russia, near Irkutsk. Applied Physics Institute, Irkutsk State University.

- MASTER-Ural: Russia, near Ekaterinburg, Since 2008. Kourovka Astronomical Observatory, Ural State University.

- MASTER-Kislovodsk: Russia, Near Kislovodsk. Kislovodsk Solar Station of the Pulkovo Observatory, Sternberg Astronomical Institute, Lomonosov Moscow State University.

- MASTER-SAAO: South Africa, Sutherland, since 2014. South African Astonomical Observatory (SAAO).

- MASTER-IAC: Spain, Canarias Islands, since 2015 The Instituto de Astrofísica de Canarias (IAC).

- MASTER-OAFA: Argentina, since 2012 Observatorio Astronomico Felix Aguilar (OAFA) , Instituto de Ciencias Astronomicas de la Tierra y del Espacio (ICATE), National University of San Juan.

- MASTER-Progenitor: Russia, Moscow, Alexander Krylov Observatory, Since 2002.

Figure 13 shows the position of MASTER-Net sites (http://observ.pereplet.ru/).

- Very small satellites for multifreqyency astrophysics have been discussed in this workshop by René Hudec et al. (2017). About the small satellites we can assist to a strong competition (typically for ESA missions, 60 proposals for 1 satellite), and moreover all the system is affected by funding problems.

The development of the Pico (Cube) and Nanosatellites is running at many Universities, mostly with involvement of students for evident goals of education.

The standard size for a CubeSat is 1 Liter Volume, i.e. $10 \times 10 \times 10 \mathrm{~cm}^{3}$ and typically a weight of $\sim 1.3 \mathrm{~kg}$. Multiple modules are possible, i.e. 3 Units $=3$ modules/units, i.e. $10 \mathrm{x}$ $10 \times 30 \mathrm{~cm}^{3}$, typically up to 12 Units.

The range of weight of Picosatellites is 0.1-1 kg, Femtosatellites 10-100 g, Nanosatellites $1-10 \mathrm{~kg}$, Microsatellites 10-100 kg.

Recent technological progress allows their use in any field of astrophysics. 


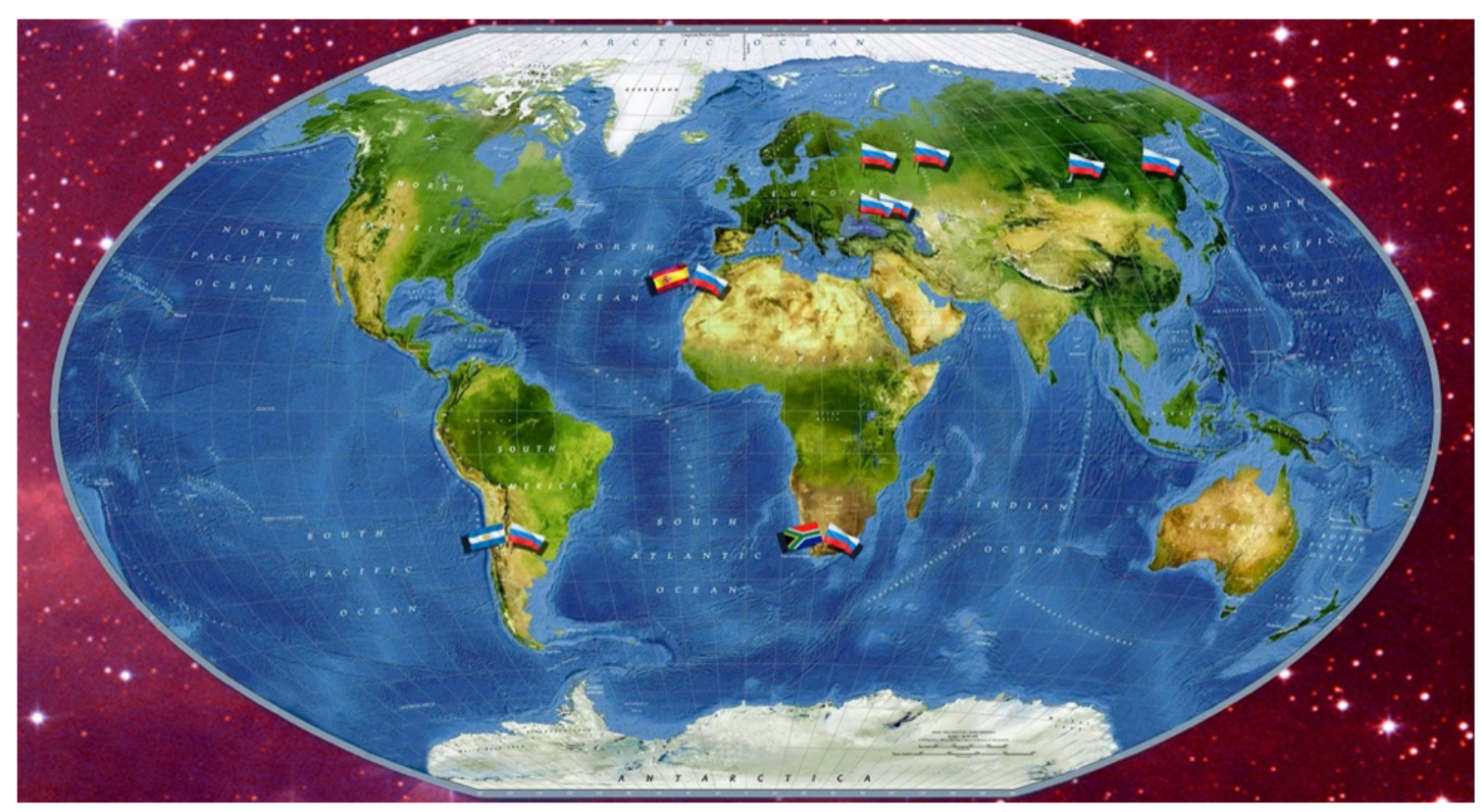

Figure 13: The MASTER-NET Robotic Autonomous Observatories worldwide (http://observ.pereplet.ru/).

Undoubtedly MASTER contributions to transient alerts in Astronomer's telegrams is fundamental. For instance in the period 2013-2014, MASTER contribution is of order 25\% of the total as shown in Fig. 14 (after Buckley, 2015).

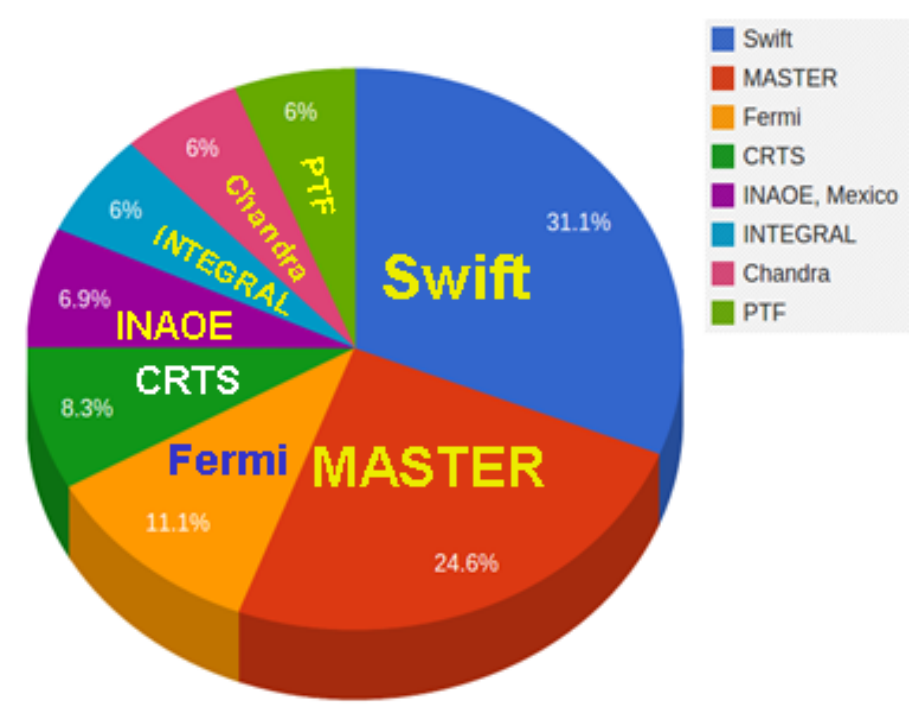

Figure 14: Contribution of different space- and ground-based experiments to the transient alerts in Astronomer's telegrams in the period 2013-2014 (after Buckley, 2015).

Though we have not shown a complete list of small experiments both space- and groundbased, we are able to affirm that small telescopes are unreplaceable tools complementary to larger telescopes and to bigger ground- and space-based multifrequency experiments. 


\section{The use of wisdom in physics}

Thanks to the numerous experiments ground- and space-based, we have collected a huge amount of experimental data, the use of which is extremely difficult. This greatly limits the possibility of reaching a synthesis. In contrast, this immense amount of data generates a production of thousands of scientific articles that only in a few cases lead to a real advancement of knowledge.

The science policy, that is now dominating the scientific world, measures the value of a researcher according to: i) the number of "scientific" publications regardless of the real contribution they make to a substantial advancement of knowledge, and ii) the number of citations.

This system foments only the increase of the production of articles at the expense of the synthesis that would be needed, and a chain of "friendly" citations.

And this produces an absurd. Indeed, in order to write a scientific paper a "normal" scientist needs roughly three months of full time work. Thus, four papers in international refereed journals is the upper limit to the yearly publication. We can be generous adding half a dozen of papers presented at the international conferences. Then the new upper limit of publication can be of order of $10 \pm 3 \mathrm{yr}^{-1}$.

Therefore we can derive a severe question to all colleagues who produce more than 13 articles/year. How they do? There are many colleagues who publish more than 100 articles per year!

One of us (FG) remember a repetitive suggestion of Livio Gratton - his professor of astrophysics at La Sapienza University of Roma - who felt a true incentive to scientific research: Favour the quality against the quantity! And he was completely right. Indeed he generated - like a supernova expelling heavy elements in the interstellar medium - a number of very famous pupils that pervaded the world of astrophysics. All the readers surely know at least two of them, sketched in Fig. 15.

After $\sim 44$ years experience about Multifrequency Astrophysics, we can affirm that: there are many problems in performing Simultaneous Multifrequency, Multisite, Multiinstrument, Multiplatform Measurements due to i) objective technological difficulties; ii) sharing common scientific objectives; iii) problems of scheduling and budgets; iv) politic management of science. In our opinion the most critic point is the latter which is moving in a "slippery ground". An example of this point is clearly illustrated by the SIXE (Spanish Italian X-ray Experiment) (Giovannelli et al., 1993). SIXE was planned as a multifrequency (X-ray, Optical) payload for Long-Term continuous observations of few selected cosmic sources in order to clearly understand the physics governing their behaviour. Later the phase-A of SIXE was completed thanks to a funding of the Spanish PNIE (Plan Nacional Investigación Espacial, being Principal Investigators Jordi Isern and Franco Giovannelli, and Lola Sabau-Graziati as first Co-I (PNIE-CICYT Report, ESP97-1784-E grant): Isern et al., 1999a; Giovannelli et al., 2002a).

Summaries of that report can be found in several papers later published (Isern et al., 1999b; Giovannelli et al., 1999a,b, 2001, 2002b). Figure 16 shows a summary of the main characteristics of SIXE.

SIXE was submitted to ASI (Agenzia Spaziale Italiana: Italian Space Agency) in order to ask a funding for sharing the costs of the payload with the PNIE, being the launcher (PEGASUS) provided by Spain. No answer at all!!! 


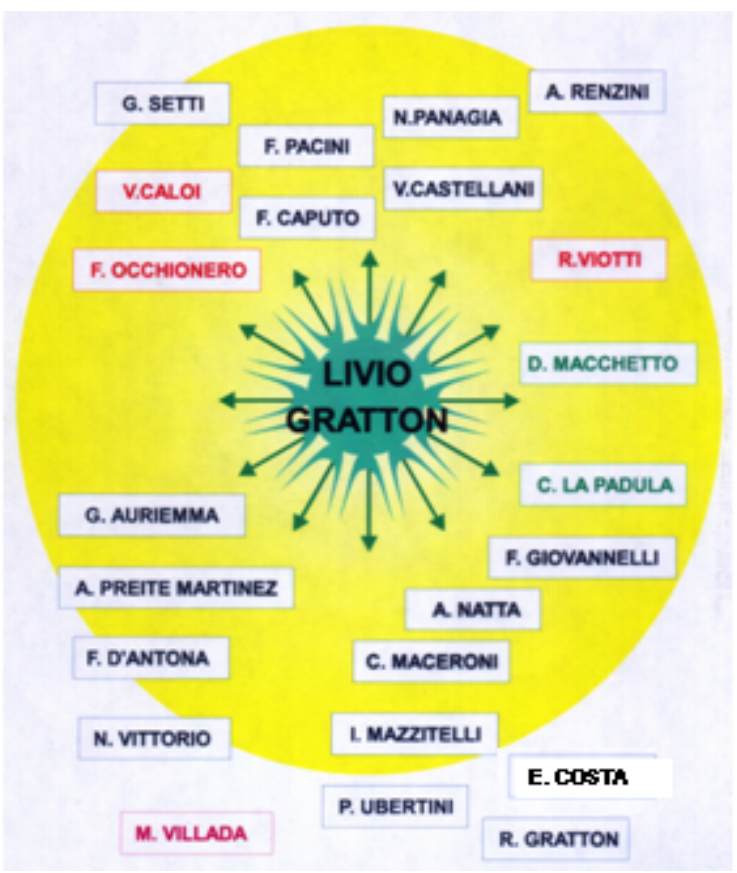

Figure 15: The supernova "Livio Gratton" produced remnant-pupils, all of them rather well known within the international astrophysical community. (Giovannelli, 2010).

\section{SIXE (Spanish Italian X-ray Experiment)}

IASF-Roma, UNI-BO, UNI-CT, UNI-PA, INAF-RM, INAF-CT, ENEA (Italy), IEEC, UPC, INTA, CNM (Spain)

FASE-A successfully concluded: funded by the Spanish PNIE (Plan Nacional Investigación Espacial)

Range X: 3-50 KeV Optical range: B-V (coaxial optical camera, type OMC of INTEGRAL) with the same F.O.V.

Platform: MINISAT-02 (Spain) Launcher: PEGASUS

\section{Philosophy: Multifequency observations (X-Optical) of FEN cosmic sources representant of homogeneous class of objects.}

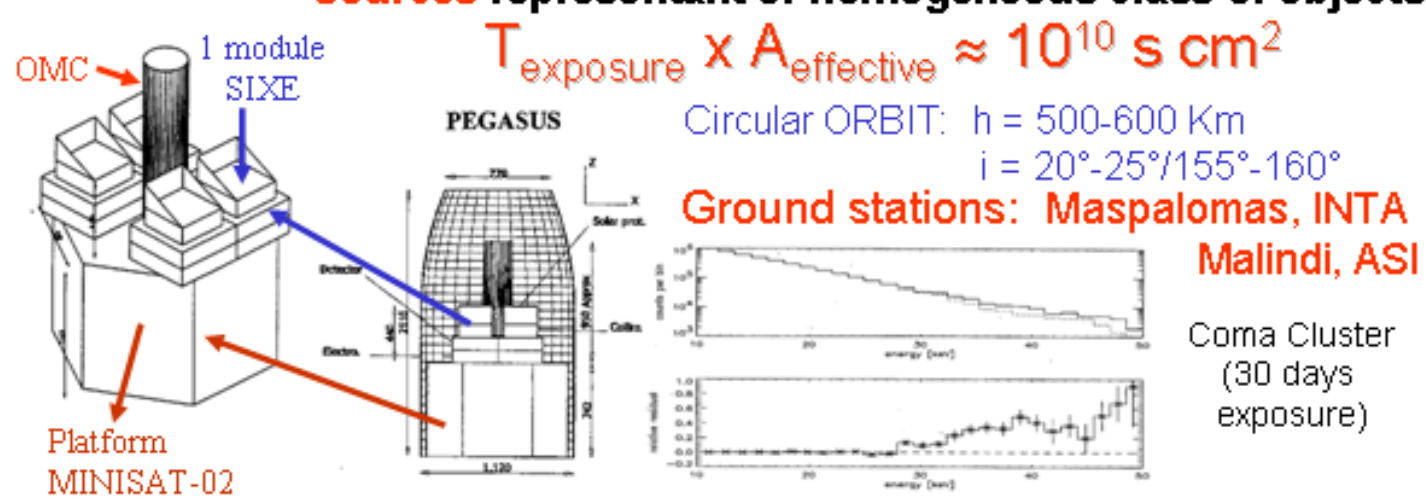

Figure 16: SIXE (Spanish Italian $\mathbf{X}$-ray Experiment): main characteristics. 
After about 25 years from the original idea (Giovannelli, F., Sabau-Graziati, L. et al., 1993) SIXE papers are still read: up till now more than 3500 readings from all the World (source: Research Gate). It is the most read paper in all INAF Institutes!!!

And now some examples of problems resolved with the help of multifrequency observations and good small quantities of wisdom in physics.

\subsection{X-ray/Be systems}

$\mathrm{X}$-ray/Be systems are formed by a compact star and an optical star. Obviously there is a mutual influence between the two stars. Low-energy (LE) processes influence high-energy (HE) processes and vice versa. Never confuse the effect with the cause. There is a general law in the Universe: Cause and Effect. The Cause generates an Effect and NOT vice versa!

Time-lag between HE events and LE events in disk-fed accreting X-ray binaries (XRBs) has been noted in many systems, but the trigger of the work resulted in a model for explaining in general such a phenomenon (Bisnovatyi-Kogan \& Giovannelli, 2017) was given by Giovannelli \& SabauGraziati (2011) who noted a systematic delay between the relative enhancement in luminosity of the optical Be star - occurring at the periastron passage of the neutron star - and the subsequent X-ray flare in the system HDE 245770/A 0535+26. The model for such a system was developed and corroborated by many events (Giovannelli, Bisnovatyi-Kogan \& Klepnev, 2013: GBK13), and later by events reported in Giovannelli et al. (2015) where also a relationship between $\Delta \mathrm{V}_{\text {mag }}$ of the optical star at the periastron and X-ray intensity $\left(\mathrm{I}_{\mathrm{X}}\right)$ of the 8-day delayed flare was produced.

Briefly, the model based on an accretion disk geometrically thin and optically thick without advection (Shakura \& Sunyaev, 1973; Bisnovatyi-Kogan, 2002) is the following: in the vicinity of periastron the mass flux $\dot{M}$ increases (depending on the activity of the Be star) between $\approx 10^{-8}$ and $\approx 10^{-7} \mathrm{M}_{\odot} \mathrm{yr}^{-1}$. The outer part of the accretion disk becomes hotter, therefore the optical luminosity $\left(\mathrm{L}_{\mathrm{opt}}\right)$ increases. Due to large turbulent viscosity, the wave of the large mass flux is propagating toward the neutron star, thus the $\mathrm{X}$-ray luminosity $\left(\mathrm{L}_{\mathrm{x}}\right)$ increases due to the appearance of a hot accretion disk region and due the accretion flow channeled by the magnetic field lines onto magnetic poles of the neutron star. The time-delay $\tau$ is the time between the optical and X-ray flashes appearance.

It is right to remind that the mechanism proposed by GBK13 for explaining the X-ray-optical delay in A $0535+26 /$ HDE 245770 is based on an enhanced mass flux propagation through the viscous accretion disk. This mechanism, known as UV-optical delay (the delay of the EUV flash with respect to the optical flash) was observed and modeled for cataclysmic variables (e.g. Smak, 1984; Lasota, 2001). Time delays have been detected also in several other X-ray transient binaries. This is the reason that urged Bisnovatyi-Kogan \& Giovannelli (2017) to generalize the aforementioned model, developed for the particular case of A 0535+26/HDE 245770 (Flavia' star). This general model provides the formula (3.1) of the time delay in transient cosmic accreting sources:

$$
\tau=6.9 \frac{\mathrm{m}^{2 / 3} \dot{\mathrm{m}}^{1 / 15}}{\alpha^{4 / 5}\left(\mathrm{~T}_{4}\right)^{28 / 15}}
$$

where:

$\mathrm{m}=\mathrm{M} / \mathrm{M}_{\odot} ; \quad \dot{\mathrm{m}}=\dot{\mathrm{M}} /\left(10^{-8} \mathrm{M}_{\odot} / \mathrm{yr}\right) ; \quad \mathrm{T}_{4}=\mathrm{T}_{0} / 10^{4} \mathrm{~K} ; \quad \alpha=$ viscosity, and

$\mathrm{T}_{0}=$ maximum temperature in optics. 
By using this formula it is possible to obtain an excellent agreement between the experimental and theoretical delays found in:

- X-ray/Be system A0535+26/HDE245770: $\tau_{\text {exp }} \simeq 8$ days (GBK13); $\tau_{t h} \simeq 8$ days;

- Cataclysmic variable SS Cygni; $\tau_{\exp }=0.9-1.4$ days (Wheatley, Mauche \& Mattei, 2003); $\tau_{t h} \simeq 1.35$ days;

- Low-mass X-ray binary Aql X-1/V1333 Aql: $\tau_{\text {exp }} \sim 3$ days (Shahbaz et al., 1998); $\tau_{t h} \simeq$ 3.2days

- Black hole X-ray transient GRO J1655-40: $\tau_{\text {exp }} \sim 6$ days (Orosz et al., 1997); $\tau_{t h} \simeq 6.5$ days.

In this general formula the $\alpha$-viscosity parameter plays an important role, and usually it is hard to be determined. However, if the other parameters are known, because experimentally determined, the formula (3.1) can be used for determining $\alpha$, taking into account the experimental delay measured in a certain source.

This general model for the time-lag for disk-fed accreting XRBs is sketched in Fig. 17.

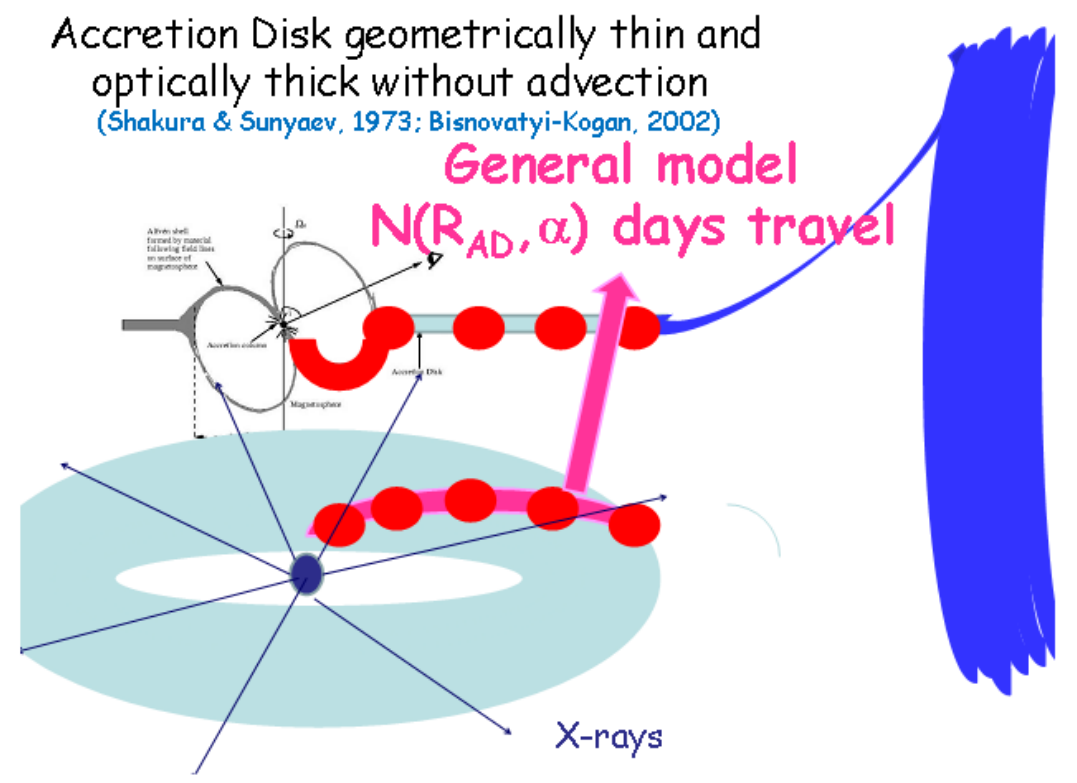

Figure 17: Time-lag general model for disk-fed accreting XRBs (Bisnovatyi-Kogan \& Giovannelli (2017).

Another example of the use of wisdom is that referred to the X-ray/Be system A 1118-61/Hen 3-640, for which Reig, Fabregat \& Coe (1997) used one single measurement of the equivalent width (EW) of $\mathrm{H}_{\alpha}(89 \AA)$ in their interesting diagram in which a relationship between the $\mathrm{H}_{\alpha}$-EWs and the orbital period $\left(\mathrm{P}_{\text {orb }}\right)$ of Be/X-ray binaries has been found. This point (red cross in Fig. 18) is clearly outside of the line best fitting the other data. However, if we use the average value (from 1985 to 1997) of $\mathrm{H}_{\alpha}-\mathrm{EW}=70 \AA$, like reported in Villada et al. (1999), also the position of the A 1118-61/Hen 3-640 system is well on the line best fitting the data (red star in Fig. 18). Moreover an indication of the possible value of the orbital period ( $\sim 350$ days), not yet known, is coming 
from that diagram. This can help the search for the orbital period of the system around the value of 350 days.

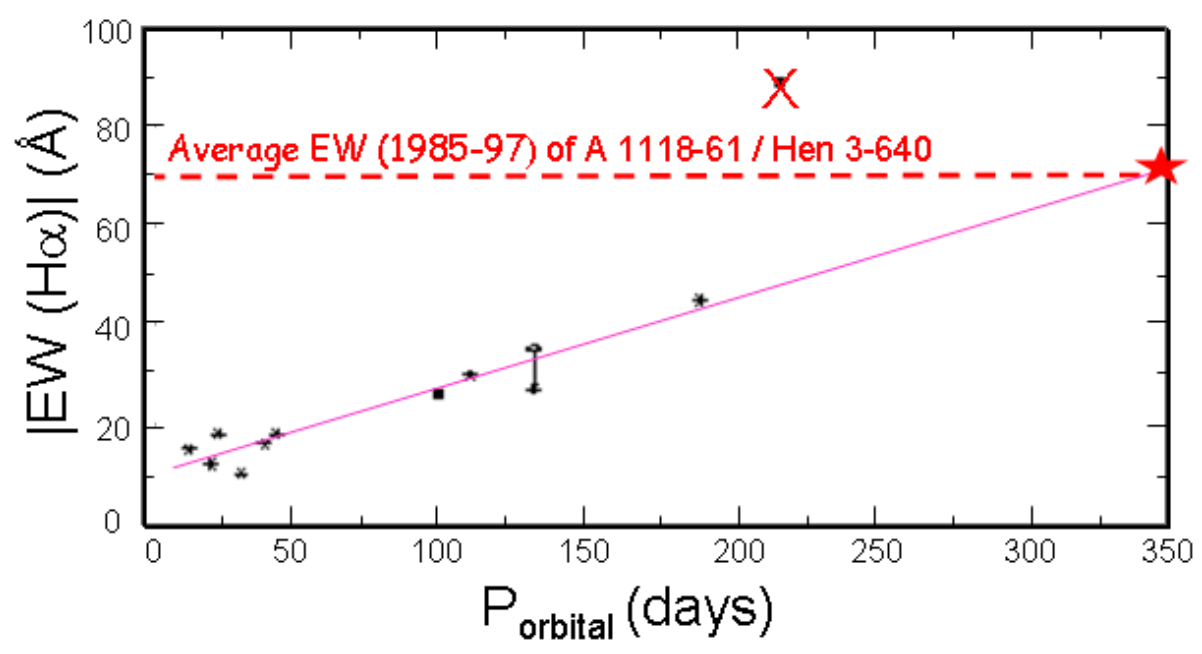

Figure 18: The relationship between $\mathrm{H}_{\alpha}$-EW and $\mathrm{P}_{\text {orb }}$ in X-ray/Be systems (after Reig, Fabregat \& Coe, 1997; Villada et al., 1999).

\subsection{The classical T Tauri star RU Lupi}

A long-term (1982-1988) multifrequency program on Classical T Tauri Stars (CTTSs) was developed by the international group leaded by Franco Giovannelli. The facilities used for such a campaign were the International Ultraviolet Explorer (IUE), the ASTRON X-ray/UV Soviet satellite, and the ESO 0.6-m UBVRI telescope, 1-m IR telescope, 1.5-m telescope for low resolution optical spectroscopy, and 3.6-m telescope for Echelle high resolution spectroscopy.

The results were published in two main papers, the first with the experimental results (Giovannelli et al., 1995), the second with the interpretation of data and modeling (Lamzin et al., 1996). A review paper about RU Lupi was published by Giovannelli (1994).

One of the main results obtained during the long-term multifrequency program was the simultaneous detection of emissions in different energy bands that allowed to construct the Spectral Energy Distribution (SED) of RU Lupi, as shown in Fig. 19.

In two occasions, RU Lupi showed a strong activity (Flare-Like Events: FLEs), much higher than that in "quiescence". Together with the FLEs reported in the literature, these two FLEs allowed to determine their periodicity: $\mathrm{P}_{\mathrm{FLEs}}=27.686 \pm 0.002$ days (Giovannelli, 1994). This could be the rotational period of RU Lupi. Indeed, if we use the relationship between the X-ray luminosity $\left(\mathrm{L}_{\mathrm{X}}\right)$ versus the rotational velocity $\left(\mathrm{v}_{\mathrm{rot}}\right)$ for $\mathrm{T}$ Tauri stars, late-type dwarfs, dKe-dMe stars, and RS CVn systems (Bouvier, 1999), the position of RU Lupi fits the relationship $\log L_{X}=27.2+2$ $\log v_{\text {rot }}$ if $P_{\mathrm{FLEs}}=27.686 \pm 0.002$ days is used, instead of using the "wrong" value reported in the literature of 3.7 days - that simply does not exist (Giovannelli et al., 1991) - whose wrong origin is largely commented in the paper by Giovannelli (1994). Figure 20 shows the diagram of the Xray luminosity $\left(\mathrm{L}_{\mathrm{X}}\right)$ versus the rotational velocity $\left(\mathrm{v}_{\mathrm{rot}}\right)$ where the "correct" position of RU Lupi is overlapped with a red cross, and the "wrong" position with the blue cross. 


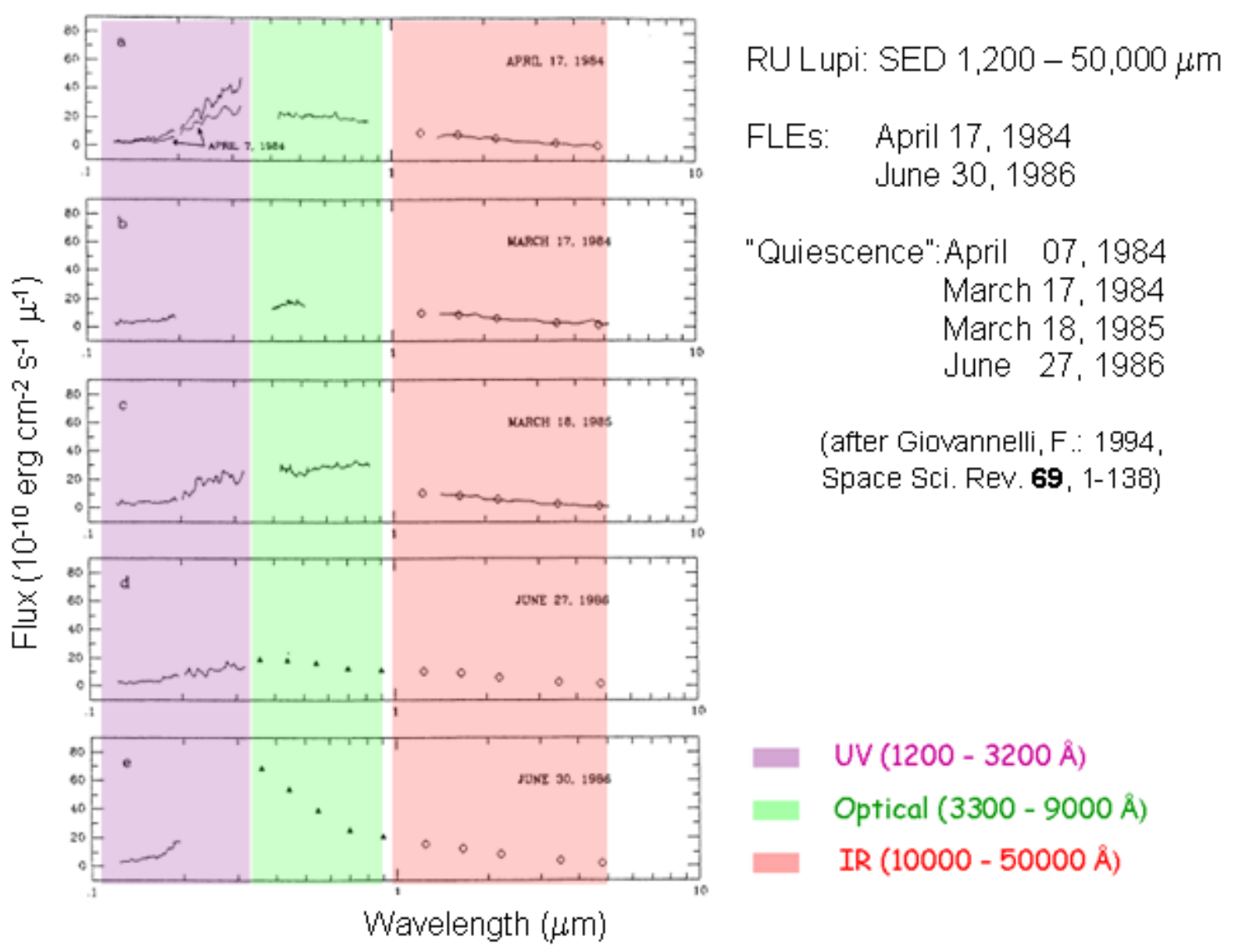

Figure 19: $\operatorname{SED}(1,200-50,000 \mu \mathrm{m})$ of RU Lupi in different epochs (after Giovannelli, 1994).

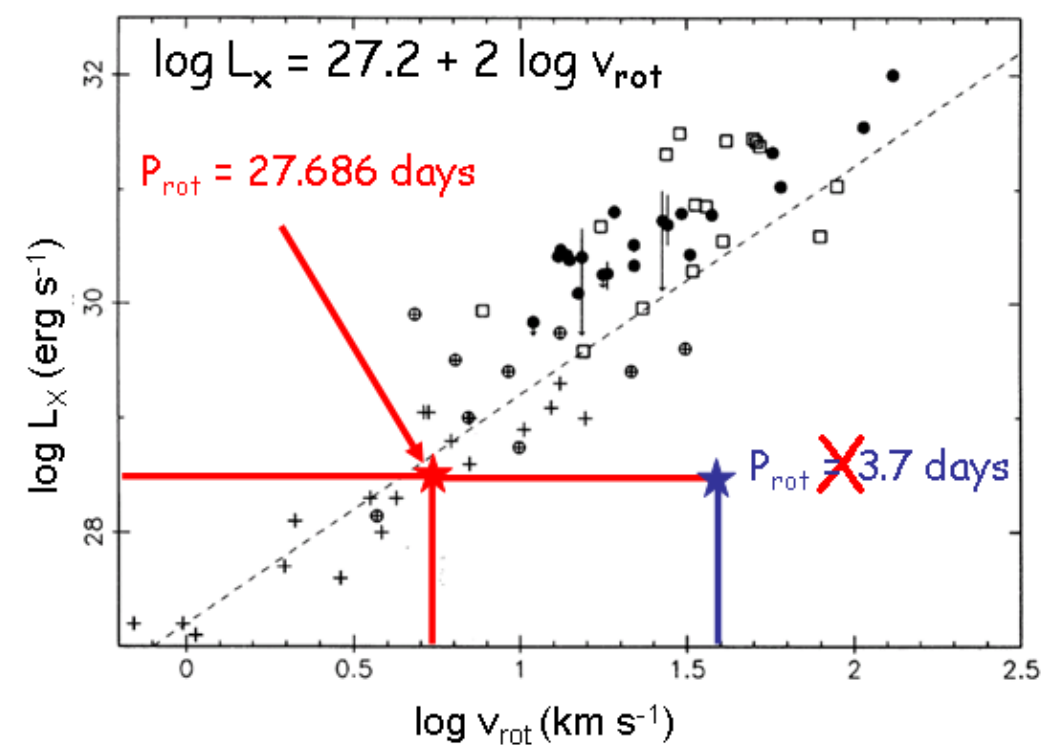

Figure 20: X-ray luminosity vs stellar equatorial velocity for TTS (•), late-type dwarfs (+), dKe-dMe stars $(\oplus)$, and RS CVn systems $(\square)$. The right position of RU Lupi is marked with a red cross, and the wrong position is marked with a blue cross (Giovannelli, 1994 after Bouvier, 1990). 


\subsection{Unified Model for Compact Sources}

The argument of the possibility of describing all the collapsed objects with a unique scheme have been discussed since long time by many authors. In their review paper, Begelman, Blandford \& Rees (1984) discussed the theory of extragalactic radio sources and in particular the unified model of active galactic nuclei (AGNs).

From the evidence that the shapes of SEDs (Spectral Energy Distributions) of different kind of AGNs (Cen A, NGC 4151, and 3C 273) are practically the same (e.g. Ramaty \& Lingenfelter, 1982), Giovannelli \& Polcaro (1986) (GP86), by using experimental data coming from the EINSTEIN observatory, constructed the maximum luminosity diagram for extragalactic objects, independent of the current classification of those objects. Indeed, those extragalactic objects have the same engine producing energy (supermassive black hole with accretion disk and jet) and they are classified as blazars, or radio-loud QSOs, or radio galaxies depending on the angle between the line of sight and the jet axis. The attenuation in the emission of a cosmic source containing a black hole in function of such an angle and the beam Lorentz's factor of the particles have been calculated by Bednarek et al. (1990).

The emission of the extragalactic X-ray sources can be expressed as $\mathrm{L}_{\mathrm{TOT}}=\mathrm{L}_{\mathrm{NUC}}+\mathrm{L}_{\mathrm{HG}}$, where, $\mathrm{L}_{\mathrm{NUC}}$ is the nuclear luminosity and $\mathrm{L}_{\mathrm{HG}}$ is the host galaxy luminosity, formed by the integrated emission of its discrete sources. Such components can be derived by using the GP86 diagram. In the long review paper by Giovannelli \& Sabau-Graziati (2004: GSG2004) there is a discussion about the GP86 diagram.

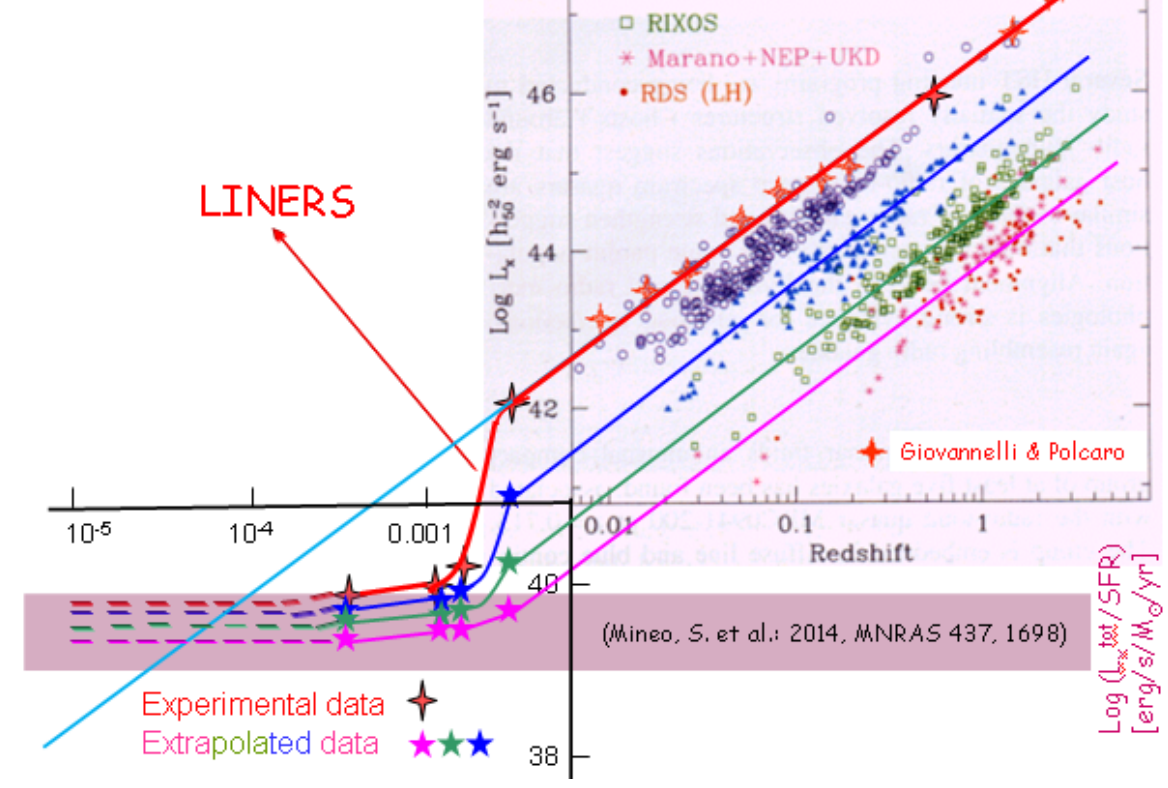

Figure 21: $L_{\mathrm{x} m a x}$ versus z for extragalactic X-ray emitters. Red crosses and red line represent GP86 diagram. The deeper surveys (Hasinger, Miyaji \& Schmidt, 2000) shown in the diagram are indicated with different colors. The light plum-colored band indicates the range of Mineo et al. (2014) results.

GP86 found a general relationship between $\log \mathrm{z}$ and the $\operatorname{logarithm}$ of the $2 \mathrm{keV}$ equivalent 
monochromatic luminosity of the brightest object in a given redshift interval (regardless of the class of the object). This function is almost constant for $\mathrm{z}<0.001$; it has a continuous inflection of about two orders of magnitude between $\mathrm{z}=0.001$ and $\mathrm{z}=0.01$, and at $\mathrm{z}>0.01$, where only AGNs are present, it matches with a straight line, with slope equal to 1.6. They suggested that such a smooth function implies a physical continuity of these objects.

Later, with the advent of higher sensitivity X-ray experiments, different samples of extragalactic X-ray emitters were discovered (Hasinger, Miyaji \& Schmidt, 2000, and the references therein). Figure 21 shows all these samples with the maximum X-ray luminosity function found by GP86 (red line). For the weaker sources, the luminosity functions are parallel to the GP86 function (blue, green, and fuchsia lines). For $\mathrm{z}<0.001$, there is a substantial constancy of the functions. In other words this means that the contribution to the luminosity is mostly coming from the discrete sources of the host galaxies. This is in complete agreement with the results obtained by Mineo et al. (2014) (light plum-colored band). The light blue line in continuation of the red line indicates the contribution of the nucleus ( $\mathrm{L}_{\mathrm{NUC}}$ ) to the total luminosity of galaxies with $\mathrm{z} \lesssim 0.002$.

\subsection{Great example of synergy between Astrophysics and History}

Bernd Aschenbach modified Sedov's relation for determining the age of a SNR (Aschenbach, 2016). He used as test the SNR Vela Jr (RX 0852.0-3946) - discovered during the ROSAT AllSky-Survey in X-rays (Aschenbach, 1998) - and he gave an age of $\mathrm{T}_{\text {Aschenbach }} \sim 725 \mathrm{yr}$ - contrary to $\mathrm{T}_{\text {Sedov }} \sim 1714 \mathrm{yr}-$ and a distance of $\sim 386 \mathrm{pc}$.

Historical document (Tatsunokuchi Persecution of Nichiren Daishonin "the Buddha of the last day of the law") supports this result with an exceptional precision: The date of the explosion was 12 September 1271 ( $1 \pm 2$ a.m. - between the hours of the rat and the ox) (Soka Gakkai, The writings of Nichiren Daishonin Vol. I, p. 196). How it is possible to affirm that the explosion of the SN Vela Jr happened in that date with a strong precision?

The answer can be found in the writings of Nichiren Daishonin. This buddhist monk presented to the public authority the "Risho Ankoku Ron" (Establishing the Correct Teaching for the peace in the country) three times: a strong and clear critic to the behaviour of authority. For this reason he was persecuted and sentenced to death.

At the moment Nichiren was about to be beheaded, a luminous object (full Moon) shot across the sky, brightly illuminating the surroundings. The executioner fell on his face, his eyes blinded. The soldiers were filled with panic. Terrified, the soldiers called off the execution. This happened on the twelfth day of the ninth month of 1271, between the hours of the rat and the ox (11:00 p.m. to 3:00 a.m.). The event culminated $10^{\circ}$ above the horizon, celestial declination $-46^{\circ}$ (position of Vela Jr).

\subsection{Some notes on relativistic jets}

Every object rotating with adequate energy produces a jet. Relativistic jets have been found in numerous galactic and extragalactic cosmic sources at different energy bands. They can be formed by electrons and protons - accelerated up to relativistic energies - which through interactions with the matter and/or photons generate high energy radiation. The spectra of such a radiation are strongly dependent on the angle formed by the beam axis and the line of sight, and obviously by 
the Lorentz factor of the particles (e.g. Bednarek et al., 1990 and the references therein; Beall, Guillory \& Rose, 1999; Beall, 2002, 2003; Beall et al., 2006, 2007).

Jets are thought to be produced by the powerful electromagnetic forces created by magnetized gas swirling toward a collapsed object (i.e. black hole). Although most of the material falls into the collapsed object, some can be ejected at extremely high speeds. Magnetic fields spun out by these forces can extend over vast distances and may help explain the narrowness of the jet (e.g. Clarke et al., 2008).

However, highly collimated supersonic jets and less collimated outflows are observed to emerge from a wide variety of astrophysical objects. They are seen in young stellar objects (YSOs), protoplanetary nebulae, compact objects (like galactic black holes or microquasars, and X-ray binary stars), and in the nuclei of active galaxies (AGNs). Despite their different physical scales (in size, velocity, and amount of energy transported), they have strong morphological similarities. What physics do they share? These systems are either hydrodynamic or magnetohydrodynamic (MHD) in nature and are, as such, governed by non-linear equations. An important review on this topic was published by de Gouveia dal Pino (2005). Very interesting discussion has been published about the role of magnetic reconnection on jet/accretion disk systems, valid in different kind of cosmic sources, like from microquasars to low luminous AGNs, till YSOs (de Gouveia Dal Pino, Piovezan \& Kadowaki, 2010).

Astrophysical jets are a remarkable laboratory for a number of important physical processes. They provide a confirmation of special relativity in terms of relativistic Doppler boosting, superluminal motion, and time dilation effects. When coupled with their black-hole/neutron-star origins, jets have implications for testing general relativity. Over the course of two decades of astrophysical research, we have become aware that jets are ubiquitous phenomena in astrophysics. Extended linear structures now associated with jets can be found in star-forming regions, galactic binaries, microquasars, active galaxies and quasars, clusters of galaxies, and $\gamma$-ray bursts. The presence and evolution of these jet-like structures is of course a testament to the principle of conservation of angular momentum.

The association of jets with accretion disks strengthens the case for similar physical processes in all these phenomena (e.g., Beall, 2003; Marscher, 2005), and it has become plausible that essentially the same physics is working over a broad range of temporal, spatial, and luminosity scales. Jets have, therefore, become a 'laboratory' or perhaps an anvil, that we can use to help us forge our understanding of the physical processes in the sky.

\section{The present situation about the knowledge of the physics of our Universe}

Undoubtedly the advent of new generation experiments ground-and space-based have given a strong impulse for verifying current theories, and for providing new experimental inputs for developing a new physics for going, probably, over the standard model (SM). Recent results coming from Active Physics Experiments (APEs) and Passive Physics Experiments (PPEs) have opened such a new path.

An extensive review on the situation about the knowledge of the physics of our Universe has been recently published by Giovannelli \& Sabau-Graziati (2016a). The reader interested is invited 
to look at that paper. However, we are obliged to discuss a few topics that, in our opinion, could be useful for a better understanding of the open problems still existing in the modern astrophysics.

\subsection{Active physics experiments}

The Large Hadron Collider (LHC) can be considered as the eighth-wonder of the world. The fields of investigation with LHC are Dark Energy, Dark Matter, Extra Dimensions, Higgs, and Supersymmetry. In this sense we can consider LHC as the vessel sailing the Dark Energy and Dark Matter unknown oceans. LHC is a complementary tool for HE observatories looking directly at the Universe. LHC is probably the highest and ultimately active-physics technological wonder, difficult to be outdated because of dimensions and costs. Probably in the next decades it will be cheaper to develop more sensitive passive-physics ground-based experiments, and even if space-based or Moon-based.

One of the most exciting results from LHC is the detection of the Higgs boson which is often called "the God particle" because it's said to be what caused the "Big Bang" that created our Universe. Matter obtains mass interacting with Higgs field. Thus, if the Higgs Boson is detected, the Standard Model of Physics would be completed.

The Standard Model of particle physics takes quarks and leptons to be fundamental elementary particles, and describes the forces that govern their interactions as mediated through the exchange of further elementary particles. The exchanged particles are photons in the case of the electromagnetic interaction, $\mathrm{W}$ and $\mathrm{Z}$ bosons in the case of the weak interaction, and gluons in the case of the strong interaction. After the discovery of the W and Z bosons in the early 1980s, the elucidation of the mechanism by which they acquire mass became an important goal for particle physics. Within the Standard Model the $\mathrm{W}$ and $\mathrm{Z}$ bosons have masses generated via the symmetry breaking Englert-Brout-Higgs-Guralnik-Hagen-Kibble mechanism, proposed in 1964 and giving rise to a massive scalar particle, the Standard Model Higgs boson (Jakobs \& Seez, 2015).

The hunt to Higgs boson - often called "the God particle" because it's said to be what caused the "Big Bang" that created our Universe: matter obtains mass interacting with Higgs field - started a few years ago with the most powerful accelerators constructed in the world, in particular with the different experiments of the LHC. These experiments can provide information about the first moment of the life of the Universe. LHC is a complementary tool for HE observatories looking directly to the Universe.

The Higgs boson discovery was announced by the ATLAS and CMS collaborations on 4th July 2012. Evidence for a new particle with the mass of about $125 \mathrm{GeV}$ and the properties of the Standard Model Higgs boson.

From ATLAS results, a $5.0 \sigma$ excess at $\sim 126.5 \mathrm{GeV}$ has been detected. This value is compatible with the expected mass of Higg's boson (Gianotti, 2012; Aad et al., 2012). The Compact Muon Solenoid (CMS) experiment at LHC detected a new boson at $125.3 \pm 0.6 \mathrm{GeV}$ with $4.9 \sigma$ significance (Incandela, 2012; The CMS Collaboration, 2012a). This result, together with that from ATLAS, if confirmed, would complete the SM of physics. Figure 22 shows the results from ATLAS and CMS (Jakobs \& Seez, 2015).

Thanks to collisions at $13 \mathrm{TeV}$ the experiment Large Hadron Collider beauty ( $\mathrm{LHCb}$ ) at LHC detected a new particle: the Pentaquark. The existence of the pentaquark was theoretically suggested since 1960-ies (Gell-Mann, 1964). Pentaquark gives a new way for the combination of the 

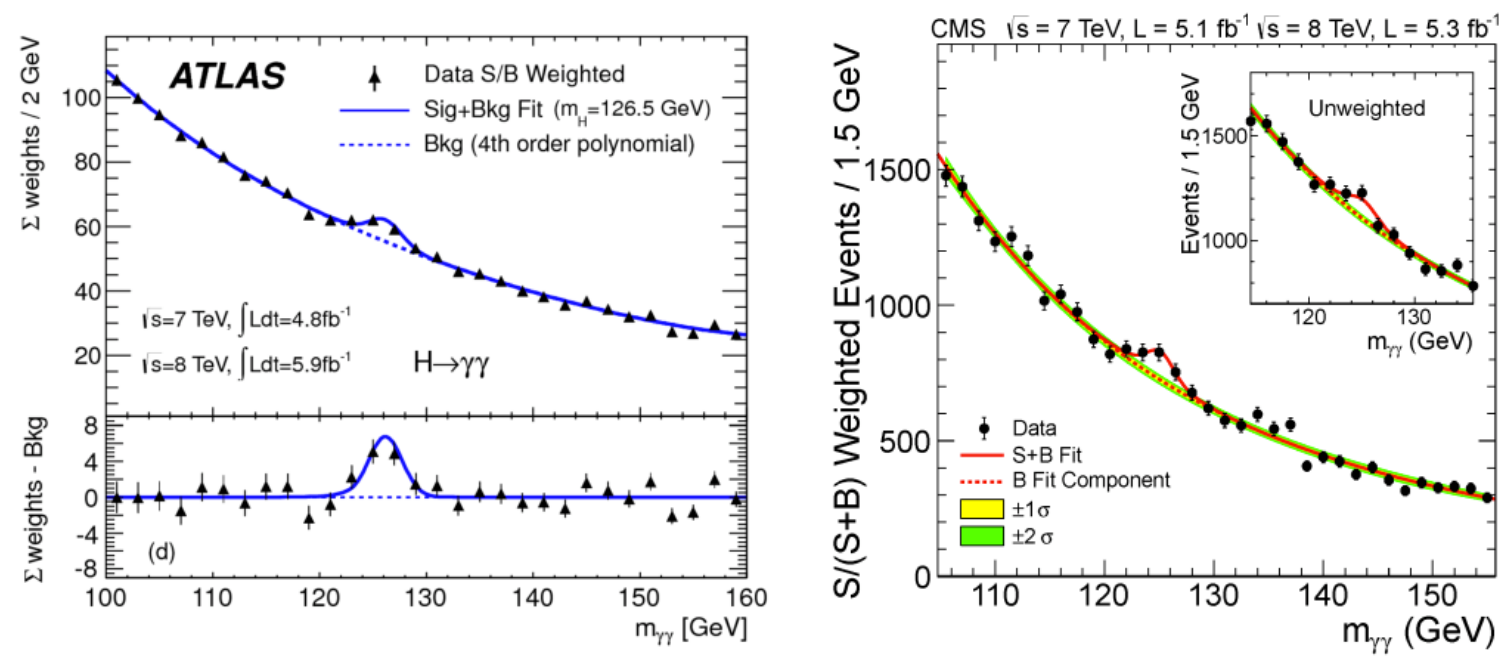

Figure 22: ATLAS and CMS results for the probable Higgs boson (adopted from Jakobs \& Seez (2015), after The ATLAS Collaboration (2012), and The CMS Collaboration (2012b).

quarks that are the fundamental constituents of neutrons and protons (Cardini, 2015; Aaij et al., 2015).

\subsection{Passive physics experiments}

One of the most important question still open is the search for experimental proof of the inflation. The expansion is thought to have been triggered by the phase transition that marked the end of the preceding grand unification epoch at $\approx 10^{-36} \mathrm{~s}$ after the Big Bang. It is not known exactly when the inflationary epoch ended, but it is thought to have been between $\approx 10^{-33}$ and $\approx 10^{-32}$ $\mathrm{s}$ after the Big Bang. The experimental proof of the inflation could come from measurements of Cosmic Microwave Background (CMB) polarization. Winstein $(2007,209)$ discussed the problem of CMB polarization in the following decade.

We know from the theory that linear polarization of the CMB photons is induced via Thomson scattering by quadrupole anisotropy at recombination that occurred at $\mathrm{z} \sim 1100$ corresponding to $\mathrm{t} \sim 1.2 \times 10^{13} \mathrm{~s}$ after the Big Bang. In turn, quadrupole anisotropy is induced by: i) density perturbations (scalar relics of inflation) producing a curl-free polarization vector field (E-modes); ii) gravitational waves (tensor relics of inflation) producing both curl-free and curl-polarization fields (B-modes).

No other sources for a curl-polarization field on the CMB at large angular scales exist. Thus, B-modes are a clear signature of inflation (e.g. de Bernardis, 2014).

Recently the collaboration of the BCEP2 experiment claims the detection of E-mode (Crites et al., 2015) and B-mode polarization of the CMB at at 7.0 $\sigma$ significance (Ade et al., 2015). If Bmode polarization would be confirmed, the inflationary model of the Universe would be definitively confirmed. However, big discoveries need big confirmations. For a robust detection of B-modes, independent measurements and precise measurements of polarized foregrounds are mandatory.

Indeed, a key element to the primordial interpretation advanced by the BICEP2 team was excluding an explanation based on polarized thermal dust emission from our galaxy (Bucher, 2015). 
An independent analysis cast doubt on the BICEP2 claim (Flauger, Hill \& Spergel, 2014). In September 2014 the Planck team published a paper on the level of polarized dust emission measured across the whole sky, and in particular in the BICEP2 field (Planck Collaboration, 2014). This work also extrapolated the polarized dust signal seen in the Planck $353 \mathrm{GHz}$ map (a frequency in the Wien tail of the CMB blackbody where dust dominates) down to $150 \mathrm{GHz}$ and reached the conclusion that the BICEP B mode signal could be entirely explained by polarized dust emission although a primordial B mode contribution could not be ruled out.

However, the theory of inflation is criticized by Ijjas, Steinhadt \& Loeb (2013) after Planck2013 results. They suggest that the origin of the Universe is not the Big Bang, but could be a "bouncing" Universe that does not need the inflation. Membrane-Universes that clashed endlessly could be a "plausible" alternative model for the Universe (Erickson et al., 2007; Steinhardt, Turok \& Starkman, 2008). Cyclic models of the universe have the advantage of avoiding initial conditions problems related to postulating any sort of beginning in time (Ijjas, 2016).

For all these reasons is even more important to find an experimental proof of the Inflation.

\subsection{Confirmation of the General Relativity}

In the last few years two further experimental results confirmed the validity of the General Relativity.

\subsubsection{Gravitational lenses}

Kochanek (2003) discussed "The whys and hows of finding 10,000 lenses", mentioning the first radio lens survey - the MIT - Green Bank survey (MG) - that found lenses by obtaining Very Large Array (VLA) snapshot images of flux-limited samples of $5 \mathrm{GHz}$ radio sources. The Hubble Space Telescope (HST), and Chandra observations (e.g. Dai, X. \& Kochanek, C.S., 2005) showed without any doubt that the gravitational lensing is operating.

Gravitational lensing is widely and successfully used to study a range of astronomical phenomena, from individual objects, like galaxies and clusters, to the mass distribution on various scales, to the overall geometry of the Universe (Williams \& Schechter, 1997). They describe and assess the use of gravitational lensing as "gold standards" in addressing one of the fundamental problems in astronomy, the determination of the absolute distance scale to extragalactic objects, namely the Hubble constant.

Several papers have been published about the strong gravitational lensing (e.g. Tyson, Kochanski \& Dell'Antonio, 1998; Tyson, 2000 and references therein), and the weak gravitational lensing (Wittman et al., 2000). A review on "Gravitational Lenses" have been published by Blandford \& Kochanek, 2004). A book on "Gravitational Lensing: Strong, Weak and Micro" was published by Meylan et al. (2006). Winn, Rusin \& Kochanek (2004) reported the most secure identification of a central image, based on radio observations of PMN J1632-0033.

Therefore, one more dowel support the General Relativity.

\subsubsection{Gravitational waves}

The Universe that contains by definition all the matter or all the energy available showed one important event that was possible to be detected on the Earth. This event was a further direct experimental demonstration of the validity of the General Relativity. Indeed, on September 14, 2015 
at 09:50:45 UTC the two detectors of the Laser Interferometer Gravitational-Wave Observatory (LIGO) simultaneously observed a transient gravitational-wave signal. It matches the waveform predicted by general relativity for the inspiral and merger of a pair of black holes and the ringdown of the resulting single black hole. The signal was observed with a significance $\geq 5.1 \sigma$. The source lies at a luminosity distance of $410_{-180}^{+160} \mathrm{Mpc}$ corresponding to a redshift $\mathrm{z}=0.090_{-0.04}^{+0.03}$. In the source frame, the initial black hole masses are $36_{-4}^{+5} \mathrm{M}_{\odot}$ and $29 \pm 4 \mathrm{M}_{\odot}$, and the final black hole mass is $62 \pm 4 \mathrm{M}_{\odot}$ with $3.0 \pm 0.5 \mathrm{M}_{\odot} \mathrm{c}^{2}$ radiated in gravitational waves. All uncertainties define $90 \%$ credible intervals. These observations demonstrate the existence of binary stellar-mass black hole systems. This is the first direct detection of gravitational waves and the first observation of a binary black hole merger (Abbott et al., 2016a).

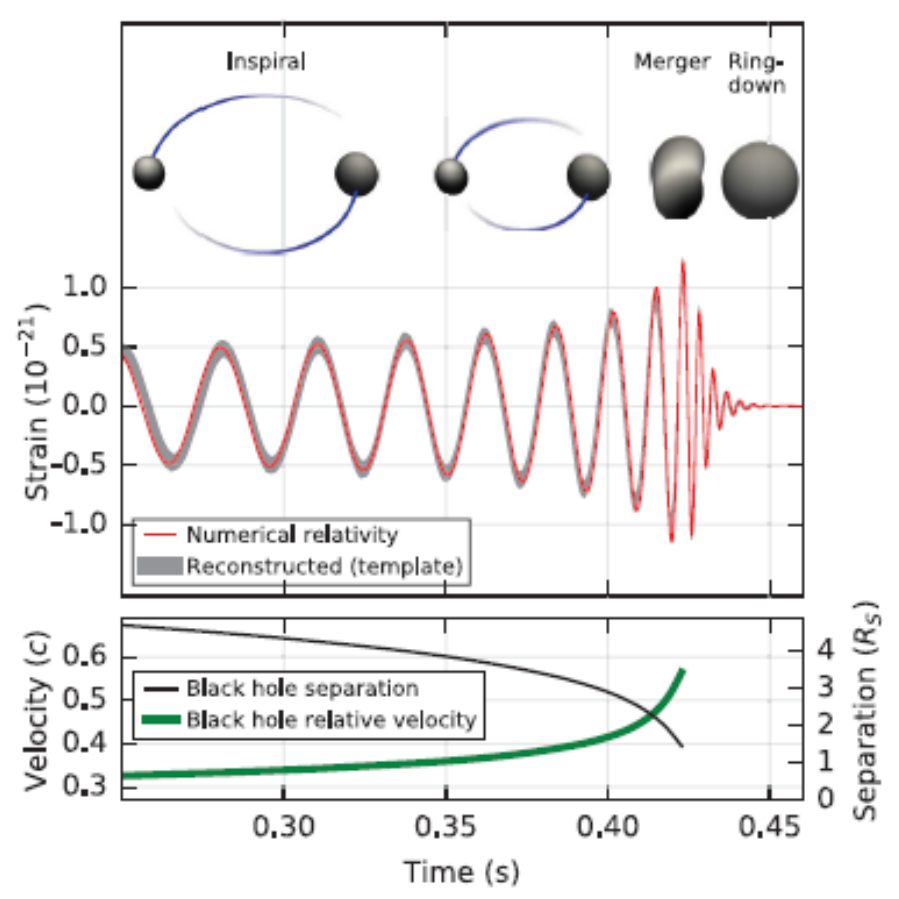

Figure 23: The GW150914 event. Top: estimated gravitational-wave strain amplitude. Bottom: the Keplerian effective black hole separation in units of Schwarzschild radii (adopted from Abbott et al., 2016a).

Abbott et al. (2016b) reported the second observation of a gravitational-wave signal produced by the coalescence of two stellar-mass black holes. The signal, GW151226, was observed by the twin detectors of the LIGO on December 26, 2015 at 03:38:53 UTC. The signal was detected at significance $\geq 5 \sigma$. The inferred source-frame initial black hole masses are $14.2_{-3.7}^{+8.3} \mathrm{M}_{\odot}$ and $7.5 \pm$ $2.3 \mathrm{M}_{\odot}$, and the final black hole mass is $20.8_{-1.7}^{+6.1} \mathrm{M}_{\odot}$. We find that at least one of the component black holes has spin greater than 0.2 . This source is located at a luminosity distance of $440_{-190}^{+180}$ Mpc corresponding to a redshift $\mathrm{z}=0.09_{-0.04}^{+0.03}$. All uncertainties define a $90 \%$ credible interval. This second gravitational-wave observation provides improved constraints on stellar populations and on deviations from general relativity.

Abbott et al. (2016c) present a possible observing scenario for the Advanced LIGO (aLIGO) and Advanced Virgo gravitational-wave detectors over the next decade, with the intention of provid- 
ing information to the astronomy community to facilitate planning for multi-messenger astronomy with gravitational waves.

Gravitational waves provide a revolutionary tool to investigate yet unobserved astrophysical objects. Especially the first stars, which are believed to be more massive than present-day stars, might be indirectly observable via the merger of their compact remnants. An interesting paper by Hartwig et al. (2016) developed a self-consistent, cosmologically representative, semi-analytical model to simulate the formation of the first stars. They estimated the contribution of primordial stars to the merger rate density and to the detection rate of the aLIGO. Owing to their higher masses, the remnants of primordial stars produce strong GW signals, even if their contribution in number is relatively small. They found a probability of $\geq 1 \%$ that the current detection GW150914 is of primordial origin. The higher masses of the first stars boost their GW signal, and therefore their detection rate. Up to five detections per year with aLIGO at final design sensitivity originate from Pop III BH-BH mergers. Approximately once per decade, we should detect a BH-BH merger that can unambiguously be identified as a Pop III remnant.

During the preparation of this paper an important event of merging of two neutron stars was detected via gravitational waves (Abbott et al., 2017a). This event has opened the new era of "Multimessenger Astrophysics" (Abbott et al., 2017b). Therefore we believe necessary to mention it in the note. $\left.{ }^{*}\right)$

\subsection{The accelerating Universe}

The discovery of the accelerating expansion of the Universe is a milestone for cosmology. A very interesting paper about this argument has been published in 2011 by the "Class for Physics of the Royal Swedish Academy of Sciences" as Scientific Background on the Nobel Prize in Physics 2011. In this paper an historical journey about the last century development of cosmology is brilliantly presented.

The discovery in 1998 that the universe is speeding up and not slowing down (Riess et al. 1998; Perlmutter et al. 1999) opened a question about the the possibility of having different phases of acceleration and deceleration of the Universe along its life. Turner \& Riess (2002) from observations of SN 1997ff at $\mathrm{z} \sim 1.7$ favor the accelerating universe interpretation and provide some direct evidence that the universe was once decelerating. They show that the strength of this conclusion depends upon the nature of the dark energy causing the present acceleration. Only for a cosmological constant is the SNe evidence definitive. Using a new test which is independent of the contents of the universe, they show that the SN data favor recent acceleration $(\mathrm{z}<0.5)$ and past deceleration $(\mathrm{z}>0.5)$.

Nielsen, Guffanti \& Sarkar (2016) found marginal evidence for cosmic acceleration from type Ia Supernovae. On the contrary, Haridasu et al. (2017) found that the SN data alone indicate an accelerating Universe at more than $4.56 \sigma$ confidence level.

\footnotetext{
(*) On 2017 August 17 the merger of two compact objects with masses consistent with two neutron stars was discovered through gravitational-wave (GW170817), gamma-ray (GRB 170817A), and optical (SSS17a/AT2017gfo) observations. The optical source was associated with the early-type galaxy NGC 4993 at a distance of just $\sim 40 \mathrm{Mpc}$, consistent with the gravitational-wave measurement, and the merger was localized to be at a projected distance of $\sim 2 \mathrm{kpc}$ away from the galaxy's center (LIGO Scientific Collaboration and Virgo Collaboration, 2017).
} 


\subsection{The Big Bang Nucleosynthesis theory has been proved}

The Big Bang Nucleosynthesis (BBN) theory predits the presence of a fixed content of light elements, the temperature of the Universe inversely proportional to the typical distance between galaxy clusters: $\mathrm{T}=\mathrm{T}(0)(1+\mathrm{z})$, and the $\mathrm{CMB}$ radiation temperature of $\sim 2.7 \mathrm{~K}$.
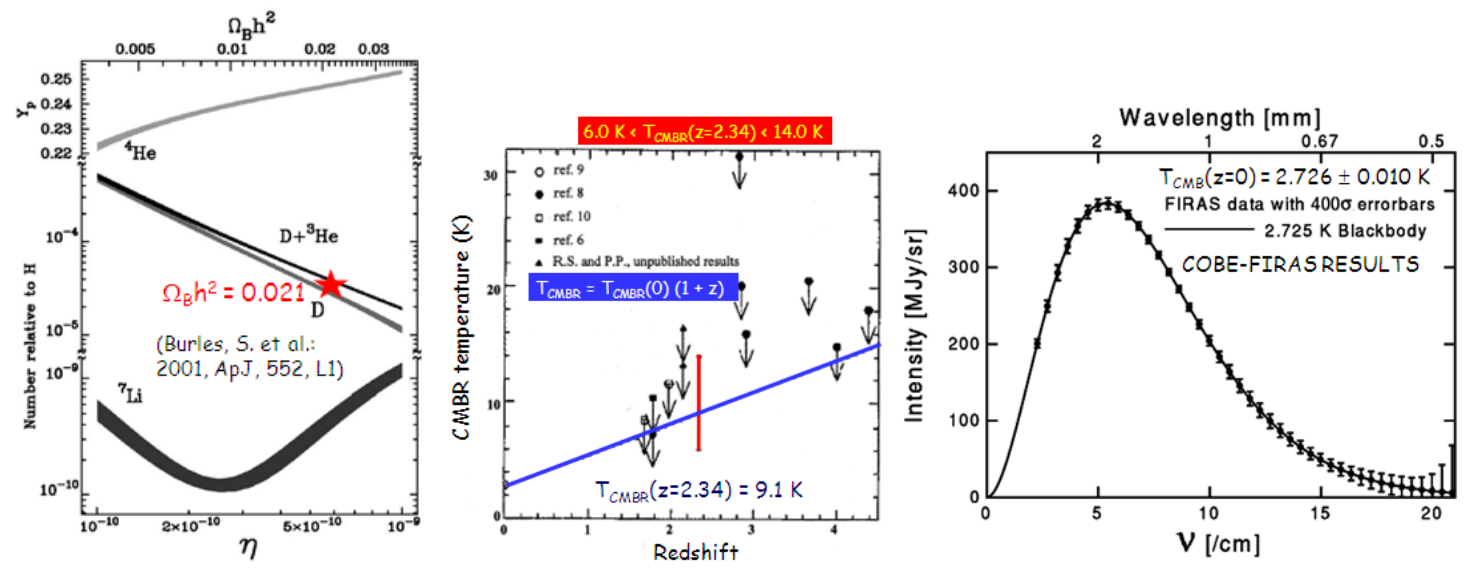

Figure 24: Three experimental results in favor of the BBN (see text for explanation).

In the last decade several experiments provided results confirming the validity of the BBN. In Fig. 24 one can see: i) (left panel): red star - the experimental confirmation of the content of the primordial light elements (de Bernardis et al., 2000) superimposed to the theoretical curves (Burles et al., 2001); ii) (middle panel): red line - the temperature of the Cosmic Microwave Background Radiation $\left(\mathrm{T}_{\mathrm{CMBR}}\right.$ ) at redshift $\mathrm{z}=2.34$, ranging between 6 and $14 \mathrm{~K}$ (Srianand, Petitjean \& Ledoux, $2000)$, in agreement with the theoretical temperature law $\mathrm{T}_{\mathrm{CMBR}}=\mathrm{T}_{\mathrm{CMBR}}(0)(1+\mathrm{z})$, which gives at $\mathrm{z}=2.34$ a temperature of $9 \mathrm{~K}$; iii) (right panel): the CMB radiation temperature $(2.726 \pm 0.010 \mathrm{~K})$ (Bartelmann, 2008, after Mather et al., 1990).

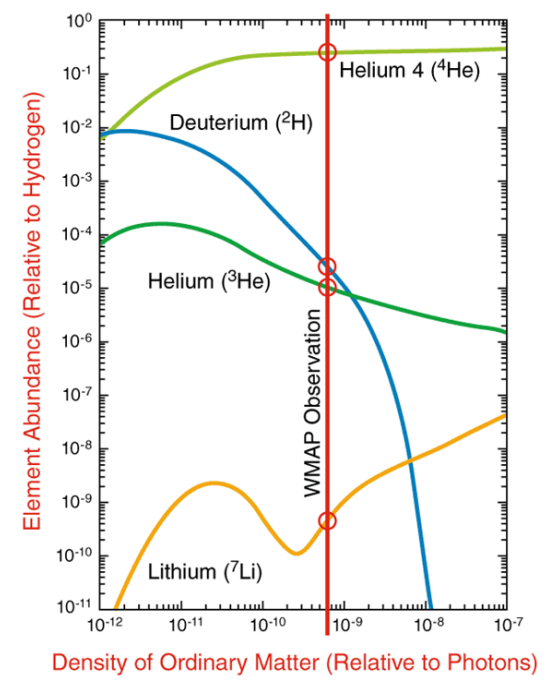

Figure 25: The experimental confirmation of the content of the primordial light elements from WMAP (https://map.gsfc.nasa.gov/universe/). 
However, the Big Bang model can be tested further, thanks to the WMAP. Given a precise measurement of the abundance of ordinary matter, the predicted abundances of the other light elements becomes highly constrained. The WMAP satellite is able to directly measure the ordinary matter density and finds a value of $4.6 \%( \pm 0.2 \%)$, indicated by the vertical red line in the Fig. 25 (https://map.gsfc.nasa.gov/universe/). This leads to predicted abundances shown by the circles in the graph, which are in good agreement with observed abundances. This is an important and detailed test of nucleosynthesis and is further evidence in support of the Big Bang theory.

\subsection{Is the Universe Flat?}

One of the most critical points about our Universe is the problem of its flatness. The present state of the cosmological tests is illustrated in Fig. 26.

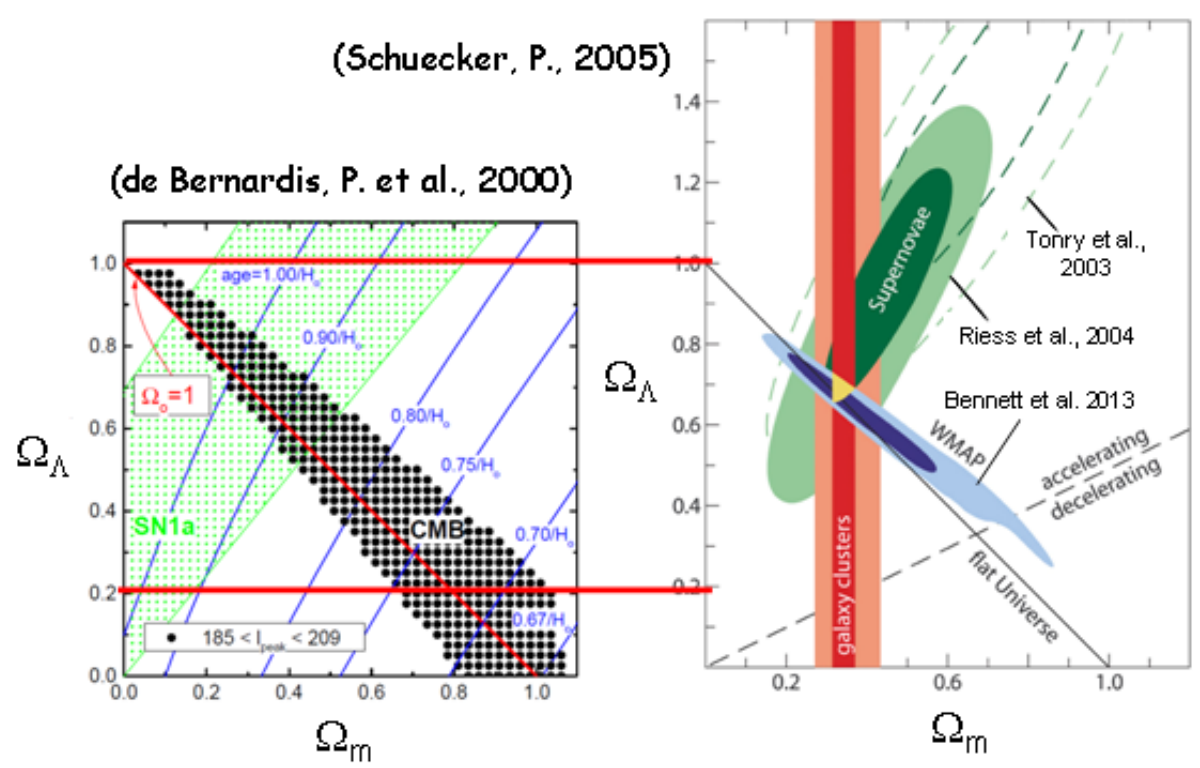

Figure 26: Constraints of cosmological parameters (after de Bernardis et al., 2000; Schuecker, 2005, Bennett et al., 2013).

The left panel of Fig. 26 shows the results obtained with the BOOMERanG (Balloon Observations Of Millimetric Extragalactic Radiation and Geomagnetics) experiment (de Bernardis et al., 2000). They are fully consistent with a spatially flat Universe. The right panel of Fig. 26 shows the combination of the likelihood contours obtained with three different observational approaches: i) type-Ia SNe (Tonry et al., 2003; Riess et al. 2004); ii) CMB (Spergel et al. 2003; Bennett et al. 2013); iii) galaxy clusters (Schuecker et al. 2003; Schuecker, 2005). One can see that the cosmic matter density is close to $\Omega_{m}=0.3$, and that the normalized cosmological constant is around $\Omega_{\Lambda}=0.7$. This sums up to unit total cosmic energy density and suggests a spatially flat universe. However, the density of cosmic matter growths with redshift like $(1+z)^{3}$ whereas the density $\rho_{\Lambda}$ related to the cosmological constant $\Lambda$ is independent of $z$. The final results from WMAP (Bennett et al., 2013) shows a little misalignment with the line of "flat Universe". Thus it is necessary to be careful in the conclusions. 


\subsection{Hubble Constant}

The Hubble constant $\left(\mathrm{H}_{0}\right)$ is one of the most important numbers in cosmology because it is needed to estimate the size and age of the universe. The important problem of determination of $\mathrm{H}_{0}$ value is one of the most exciting. Indeed, in the literature it is possible to find many determinations coming from different experiments using different methods. However, it is very complicate to obtain a true value for $\mathrm{H}_{0}$. It is necessary to have two measurements: i) spectroscopic observations that reveal the galaxy's redshift, indicating its radial velocity; ii) the galaxy's precise distance from Earth (and this is the most difficult value to determine).

A large summary about the methods used for $\mathrm{H}_{0}$ determination, and its derived values can be found in the Proceedings of the Fall 2004 Astronomy 233 Symposium on "Measurements of the Hubble constant" (Damon et al., 2004). In this book, Teymourian (2004), after a comparison of many constraints on the Hubble constant determinations, reports a value $\mathrm{H}_{0}=68 \pm 6 \mathrm{~km} \mathrm{~s}^{-1}$ $\mathrm{Mpc}^{-1}$.

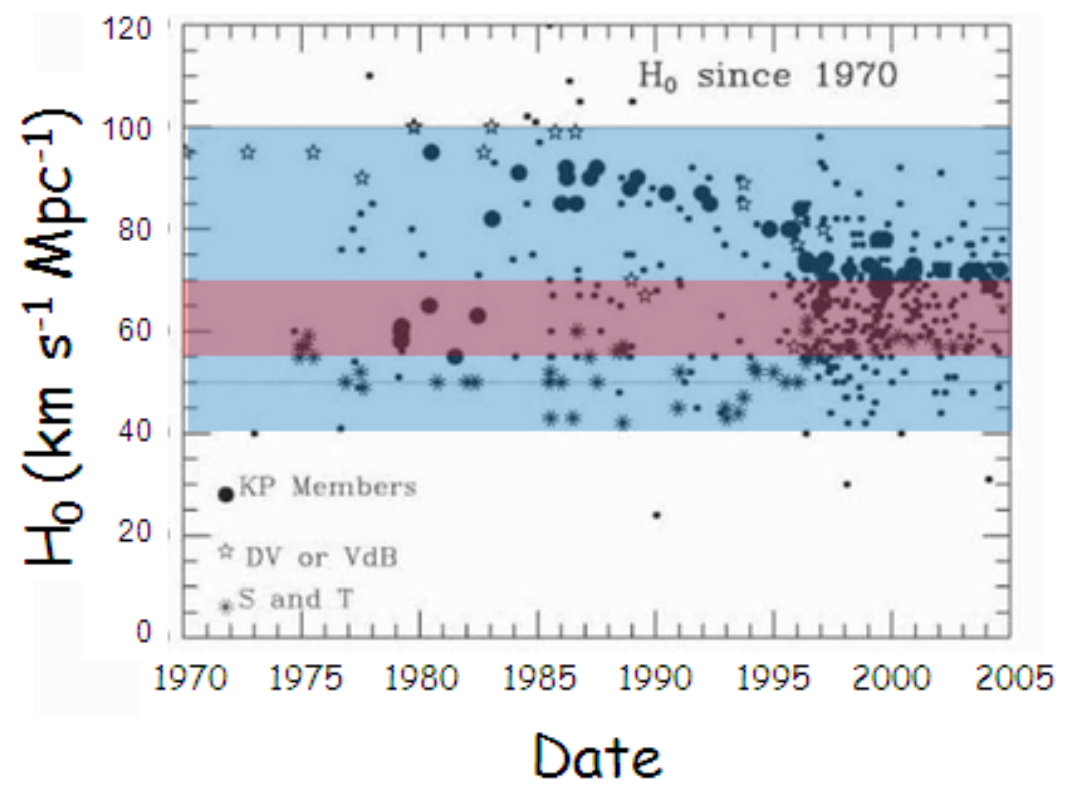

Figure 27: The Hubble constant determinations since 1970. The light-blue rectangle limits all the $\mathrm{H}_{0}$ determinations. The light-red rectangle shows the narrow limits to to which the values of $\mathrm{H}_{0}$ are converging (After John Huchra, 2008).

Freedman \& Madore (2010) published a review about The Hubble Constant in which they discuss the considerable progress made in determining the Hubble constant over the past two decades. They discuss the cosmological context and importance of an accurate measurement of the Hubble constant, focusing on six high-precision distance-determination methods: Cepheids, tip of the red giant branch, maser galaxies, surface brightness fluctuations, the Tully-Fisher relation, and Type Ia supernovae. Their best current estimate of the Hubble constant is $\mathrm{H}_{0}=73 \pm 2$ (random) \pm 4 (systematic) $\mathrm{km} \mathrm{s}^{-1} \mathrm{Mpc}^{-1}$.

A recent discussion about the Hubble constant has been published by Giovannelli \& SabauGraziati (2014a), where it is possible to find also a large number of references, reporting the many controversial evaluations of $\mathrm{H}_{0}$. 
Figure 27 shows the determinations of $\mathrm{H}_{0}$ since 1970 (adapted from John Huchra, 2008). Practically all the determinations lie in the range 40-100 $\mathrm{km} \mathrm{s}^{-1} \mathrm{Mpc}^{-1}$ (marked with light-blue), and most of them are converging in the range 55-70 $\mathrm{km} \mathrm{s}^{-1} \mathrm{Mpc}^{-1}$ (marked with light-red). Data have been collected by Huchra (2007). This precious list contains all the determinations of Hubble constant till that date (https://www.cfa.harvard.edu/ dfabricant/huchra/hubble.plot.dat). $\left.{ }^{*}\right)$

However, Riess et al. (2011) with the HST determined a value of $\mathrm{H}_{0}=73.8 \pm 2.4 \mathrm{~km} \mathrm{~s}^{-1}$ $\mathrm{Mpc}^{-1}$. This value agrees with the WMAP results: $\mathrm{H}_{0}=71.0 \pm 2.5 \mathrm{~km} \mathrm{~s}^{-1} \mathrm{Mpc}^{-1}$ (Komatsu et al., 2011). Bennett et al. (2014) discussed the progress occurred in recent years for determining the Hubble constant: results coming from the cosmic distance ladder measurements at low redshift and CMB measurements at high redshift. The CMB is used to predict the current expansion rate of the universe by best-fitting cosmological model. At low redshift baryon acoustic oscillation (BAO) measurements have been used - although they cannot independently determine $\mathrm{H}_{0}$ - for constraining possible solutions and checks on cosmic consistency. Comparing these measurements they found $\mathrm{H}_{0}=69.6 \pm 0.7 \mathrm{~km} \mathrm{~s}^{-1} \mathrm{Mpc}^{-1}$.

Does this determination, finally, close the history about the search of the "true" value of $\mathrm{H}_{0}$ ?

(*) Professor John Huchra, died unexpectedly October 8th, 2010.

\subsection{Reionization Epoch}

Ground-based observations of the CMB on subdegree angular scales suggest that the gas content of the universe was mostly neutral since recombination at $\mathrm{z} \sim 1000$ until about $\mathrm{z} \sim 100$ (Gnedin, 2000 and the references therein) because earlier reionization would have brought the last scattering surface to lower redshift, smoothing the intrinsic CMB anisotropy. At the same time, we know that the universe is highly ionized, since $\mathrm{z} \approx 5$, from observations of the spectra of quasars with the highest redshifts (e.g. Giallongo et al. 1994). This change of the ionization state of the universe from neutral to highly ionized is called "reionization". How large is the redshift to which the reionization started and stopped is object of strong debate.

The formation of the first stars and quasars marks the transformation of the universe from its smooth initial state to its clumpy current state. In current cosmological models, the first sources of light began to form at a redshift $\mathrm{z} \sim 30$ and reionized most of the hydrogen in the universe by $\mathrm{z} \sim 7$ (see review by Loeb \& Barkana, 2001).

Recently Matsuoka et al. (2016) reported the discovery of 15 QSOs and bright galaxies at $5.7<z<6.9$ from the Subaru High-z Exploration of Low-Luminosity Quasars (SHELLQs) project.

The argument for an extended period of reionization is now proved by measurements. Indeed, the WMAP has detected the correlation between temperature and polarization on large angular scales (Kogut et al., 2003) that has an amplitude proportional to the total optical depth of CMB photons to Thomson scattering, $\tau$ (Kaplinghat et al., 2003; Sunyaev \& Zeldovich, 1980; Zaldarriaga, 1997).

Modeling reionization with a single sharp transition at $z_{\mathrm{ri}}$, a multi-parameter fit to the WMAP data gives $z_{\mathrm{ri}}=17 \pm 5$ (Spergel et al., 2003). On the other hand, the evolution of quasar spectra from $\mathrm{z} \approx 7$ and $\mathrm{z} \approx 6$ shows a rapid decrease in the amount of neutral Hydrogen, indicating the end of reionization (Fan et al., 2003). A simple interpretation to explain these two very different 
datasets is that reionization started early, $\mathrm{z}_{\mathrm{ri}} \sim 20$, but did not conclude until much later $(\mathrm{z} \sim 6$ ) (Knox, 2003).

This was also confirmed by the results from Subaru Deep Field (SDF) (Kashikawa et al., 2006; Kashikawa, 2007): the reionization of the universe has not been completed at $\mathrm{z}=6.5$. Also Ota et al. (2008) in performing narrowband imaging of the SDF found two Ly $\alpha$ emitters (LAEs) at $\mathrm{z}=$ 7. This established a new redshift record, showing that galaxy formation was in progress just 750 Myr after the Big Bang. They found that the attenuation of the Ly $\alpha$ photons from LAEs by the neutral hydrogen possibly left at the last stage of cosmic reionization at $\mathrm{z} \sim 6-7$.

Ouchi et al. (2009a) suggested an existence of a well-developed ionized bubble at $\mathrm{z}=7$. Ouchi et al. (2009b) reported the discovery of a giant LAE with a Spitzer/Infrared Array Camera (IRAC) counterpart near the reionization epoch at $\mathrm{z}=6.595$. Although the nature of this object is not yet clearly understood, this could be an important object for studying cooling clouds accreting onto a massive halo, or forming-massive galaxies with significant outflows contributing to cosmic reionization and metal enrichment of intergalactic medium.

Ouchi et al. (2010) presented the Ly $\alpha$ luminosity function (LF), clustering measurements, and Ly $\alpha$ line profiles based on the largest sample to date of 207 LAEs at $\mathrm{z}=6.6$. The combination of various reionization models and their observational results about the LF, clustering, and line profile indicates that there would exist a small decrease of the intergalactic medium's (IGM's) Ly $\alpha$ transmission owing to reionization, but that the hydrogen IGM is not highly neutral at $\mathrm{z}=6.6$. Their neutral-hydrogen fraction constraint implies that the major reionization process took place at $\mathrm{z} \gtrsim 7$.

Jiang et al. (2011) presented Keck spectroscopic observations of $\mathrm{z}>6$ Lyman-break galaxy (LBG) candidates in the Subaru Deep Field (SDF). Their Ly $\alpha$ LF is also generally in agreement with the results of LAEs surveys at $\mathrm{z} \sim 5.7$ and 6.6. This study shows that deep spectroscopic observations of LBGs can provide unique constraints on both the UV and Ly $\alpha$ LFs at $\mathrm{z}>6$.

Ono et al. (2012) presented the results of their ultra-deep Keck/DEIMOS spectroscopy of zdropout galaxies in the SDF and Great Observatories Origins Deep Survey's northern field. The fractions of Ly $\alpha$-emitting galaxies drop from $\mathrm{z} \sim 6$ to 7 and the amplitude of the drop is larger for faint galaxies than for bright galaxies. These two pieces of evidence would indicate that the neutral hydrogen fraction of the IGM increases from $\mathrm{z} \sim 6$ to 7 and that the reionization proceeds from high- to low-density environments, as suggested by an inside-out reionization model.

The WMAP detection of reionization (Kogut et al. 2003) implies the existence of an early generation of stars able to reionize the universe at $\mathrm{z} \sim 20$. Panagia et al. (2005) in deep HST/VLT/Spitzer images found that the source UDF 033238.7-274839.8 - a post-starburst galaxy with a mass $\sim 6 \times 10^{11} \mathrm{M}_{\odot}$ placed at $\mathrm{z} \geq 6.5$ - may be capable of reionizing its surrounding region of the universe, starting the process at a redshift as high as $\mathrm{z}=15 \pm 5$.

The question about the end of the reionization is strongly disputed. However, in our opinion probably it is possible to put a reasonable limit to the epoch of the reionization end $(\mathrm{z} \sim 6)$, looking at the paper by Toshikawa et al. (2012). They reported the discovery of a protocluster at $\mathrm{z} \sim 6$ containing at least eight cluster member galaxies with spectroscopic confirmations in the widefield image of the SDF. They found no significant difference in the observed properties, such as Ly $\alpha$ luminosities and UV continuum magnitudes, between the eight protocluster members and the seven non-members. The velocity dispersion of the eight protocluster members is $647 \pm 124$ 


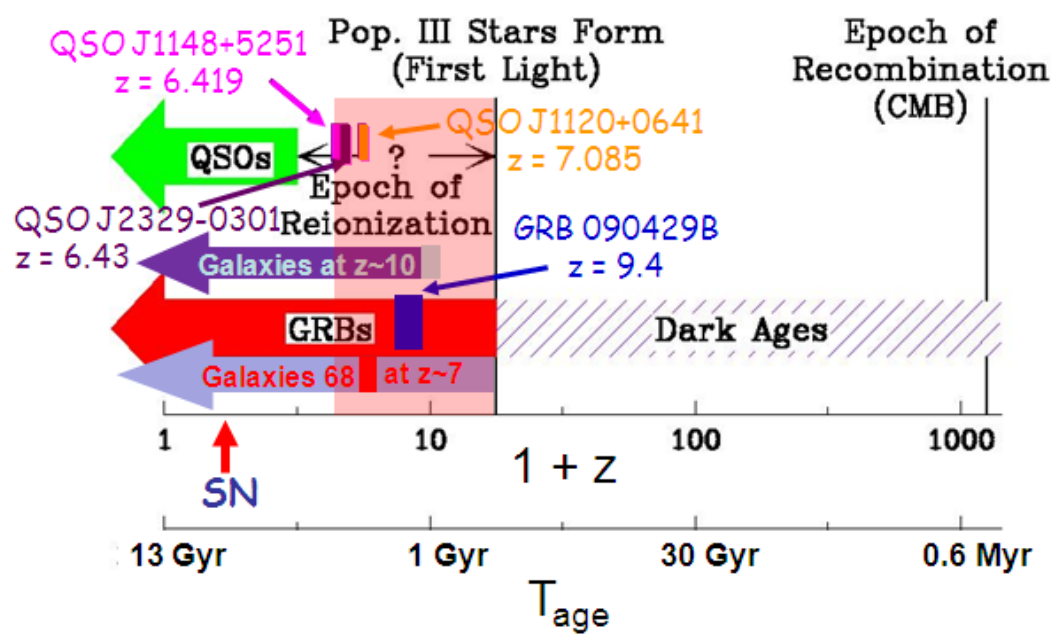

Figure 28: A sketch of reionization epoch (after Xiangping Wu's Talk at the Summer School on "Cosmic Reionization" at the KIAA-PKU , Beijing, China, July 1-11, 2008).

$\mathrm{km} \mathrm{s}^{-1}$, which is about three times higher than that predicted by the standard cold dark matter model. This discrepancy could be attributed to the distinguishing three-dimensional distribution of the eight protocluster members. They discussed two possible explanations for this discrepancy: either the protocluster is already mature, with old galaxies at the center, or it is still immature and composed of three subgroups merging to become a larger cluster. In either case, this concentration of $z=6.01$ galaxies in the SDF may be one of the first sites of formation of a galaxy cluster in the universe.

Figure 28 shows schematically the updated experimental situation about cosmic sources (galaxies, GRBs, QSOs, SNe) detected at high redshifts. The light-red rectangle marks the possible range of $\mathrm{z}$ during which the reionization occurred.

However, although there is rather good agreement about the epoch of reionization, how really reionization occurs is still object of debate. Indeed, Dopita et al. (2011), considering that recent observations show that the measured rates of star formation in the early universe are insufficient to produce reionization, suggest the presence of another source of ionizing photons. This source could be the fast accretion shocks formed around the cores of the most massive haloes.

An interesting review about The epoch of reionization was published by Zaroubi (2013).

\subsection{Background Radiation in the Universe}

Tiny inhomogeneities in the early Universe left their imprint on the microwave background in the form of small anisotropies in its temperature. These anisotropies contain information about basic cosmological parameters, particularly the total energy density and curvature of the universe.

On April 23, 1992, the COBE team announced the historical discovery of the anisotropies of cosmic microwave background radiation with characteristic anisotropy $\Delta \mathrm{T} / \mathrm{T} \approx 10^{-5}$ or $\Delta \mathrm{T} \sim 30$ $\mu \mathrm{K}$ on angular scales larger than $\sim 7^{\circ}$ at the annual meeting of American Physical Society in Washington, D.C. (Smoot et al., 1992).

Observations of the cosmic microwave background temperature anisotropies have revolutionized and continue to revolutionize our understanding of the universe. The observation of the CMB 
anisotropies angular power spectrum with its plateau, acoustic peaks, and high frequency damping tail have established a standard cosmological model consisting of a flat - critical density - geometry, with contents being mainly dark energy and dark matter and a small amount of ordinary matter. In this successful model the dark and ordinary matter formed its structure through gravitational instability acting on the quantum fluctuations generated during the very early inflationary epoch. Current and future observations will test this model and determine its key cosmological parameters with spectacular precision and confidence (see the Nobel Lecture of George F. Smoot (2007) for an exhaustive review about the Cosmic Background Radiation Anisotropies).

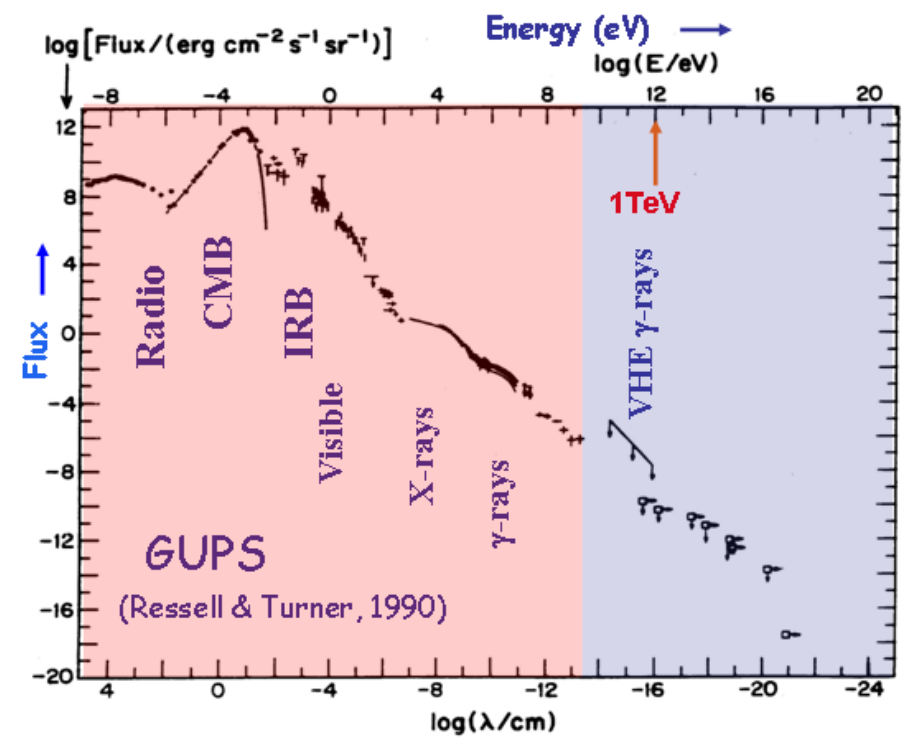

Figure 29: The Grand Unified Photon Spectrum of the Diffuse Extragalactic Background Radiation (after Ressell \& Turner, 1990).

But the cosmic background radiation, although is peaked in the microwave region, permeates through the whole electromagnetic spectrum and is known as the Diffuse Extragalactic Background RAdiation (DEBRA). It is possible to consider the DEBRA as a radiation produced by a cosmic source: the whole Universe. Such a background radiation from radio to HE $\gamma$-ray energy bands has been deeply discussed by Ressell \& Turner (1990), and in GSG2004 and the references therein. The analysis of the different components of DEBRA leads to the Grand Unified Photon Spectrum (GUPS), covering 29 orders of magnitude of the electromagnetic spectrum, from $10^{-9}$ to $10^{20} \mathrm{eV}$, as shown in Fig. 29 (after Ressell \& Turner, 1990). The light-red and the light-indico rectangles indicate the domains with energies less or greater than $\approx 10 \mathrm{GeV}$, respectively. The domain at higher energies is now explored by numerous experiments space-based, like Fermi LAT observatory (up to $300 \mathrm{GeV}$ ) and ground-based, like Whipple, Veritas, HESS, Magic, and the coming CTA (Cherenkov Telescopes Array). All these experiments will provide to fill the zone of the GUPS diagram prepared by Ressell \& Turner (1990) where only upper limits were reported.

Henry $(1999,2002)$ thoroughly discussed the experimental situation of the cosmic background till 2000.

Durrer (2015) in her interesting review describes the discovery of the cosmic microwave background radiation in 1965 and its impact on cosmology in the 50 years that followed. 


\subsubsection{Extragalactic Background Light}

The intergalactic space is filled with the light produced by all the stars and accreting compact objects that populated the observable Universe throughout the whole cosmic history. This relic cosmic background from IR to UV is called the diffuse Extragalactic Background Light (EBL), long before known as DEBRA (Ressel \& Turner, 1990).

Direct measurements of the EBL are difficult due to bright local foregrounds. A powerful approach for probing these diffuse radiation fields in the UV to far-IR bands is through $\gamma-\gamma$ absorption of high-energy photons. Actually pair production $\left(\mathrm{e}^{+} \mathrm{e}^{-}\right)$against EBL photons with wavelengths from ultraviolet to infrared is effective at attenuating $\gamma$-rays with energy above $\sim 10 \mathrm{GeV}$. This process introduces an attenuation in the spectra of $\gamma$-ray sources above a critical energy (e.g. Costamante, 2012; Buson, 2014).

The last decade has been foreboding of a full coverage of the HE-VHE $\gamma$-ray energy band, thanks to the many ground- and space-based high sensitivity experiments, as shown in Fig. 30. These experiments have provided a large amount of data from many extragalactic emitters at high redshift (e.g. Costamante, 2012). Thanks to measurements of the quasar 3C 279 ( $\mathrm{z} \simeq 0.54$ ) obtained with the MAGIC experiment (Albert et al., 2008), and with the many sources at high redshift, including Gamma Ray Bursts (GRBs) measured with the FERMI observatory (Abdo et al., 2010), it has been demonstrated that the Universe is more transparent to $\gamma$-rays than before believed (Coppi \& Aharonian, 1997).

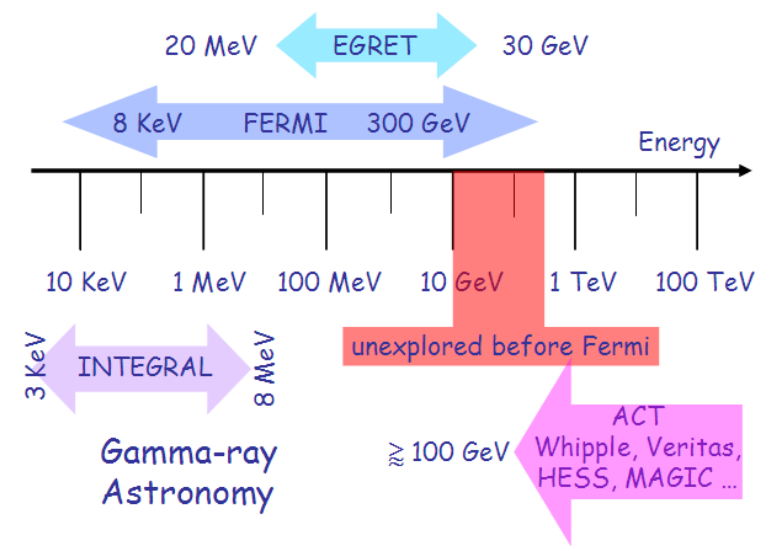

Figure 30: The HE-VHE $\gamma$-ray energy band completely explored with the new generation ground- and space-based experiments.

Domíguez et al. (2011) started from the fact that the overall spectrum of the EBL between 0.1 and $1000 \mu \mathrm{m}$ has never been determined directly from galaxy spectral energy distribution (SED) observations over a wide redshift range. They took into account a sample of about 6000 galaxies in the redshift range from 0.2 to 1 from the All-wavelength Extended Groth Strip International Survey (AEGIS) by fitting Spitzer Wide-Area Infrared Extragalactic Survey (SWIRE) templates, and calculated the evolution of the luminosity densities from the UV to the IR, the evolving star formation rate density of the Universe, the evolving contribution to the bolometric EBL from the different galaxy populations including AGN galaxies and the buildup of the EBL. Their EBL calculations were compared with those from a semi-analytic model, another observationally based model and 
observational data. The results are that the EBL is well constrained from the UV to the mid-IR, but independent efforts from IR and $\gamma$-ray astronomy are needed in order to reduce the uncertainties in the far-IR.

Cooray (2016) reviews the Extragalactic Background Light Measurements and Applications. This review covers the measurements related to the extragalactic background light intensity from $\gamma$-rays to radio in the electromagnetic spectrum over 20 decades in wavelength. Figure 31 shows such EBL measurements that updated those reported by Ressel \& Turner (1990) (Fig. 29). It is important to remark that the numerous measurements in the range of the VHE $\gamma$-rays (Log E $\approx 9-13 \mathrm{eV}$ ) have filled the zone marked with the light-indico rectangle in the Fig. 29, where no measurements or only upper limits were available in the 1990-ies.

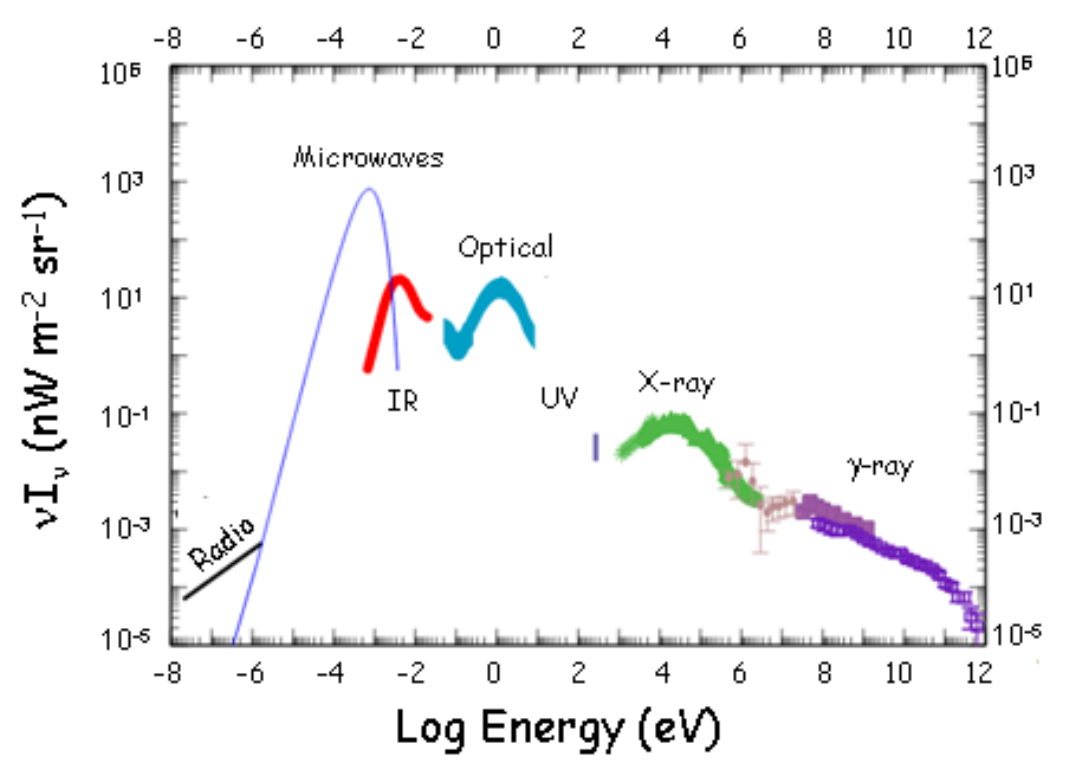

Figure 31: Intensity of the extragalactic background $\left(v \mathrm{I}_{v}\right.$ in units of $\left.\mathrm{nW} \mathrm{m} \mathrm{m}^{-2} \mathrm{sr}^{-1}\right)$ as a function of the energy (after Cooray, 2016).

The CMB remains the best measured spectrum with an accuracy better than $1 \%$. The measurements related to the Cosmic Optical Background (COB) are impacted by the large zodiacal light associated with interplanetary dust in the inner Solar System. The best measurements of COB come from an indirect technique involving $\gamma$-ray spectra of bright blazars with an absorption feature resulting from pair-production off of $\mathrm{COB}$ photons. The cosmic infrared background (CIB) established an energetically important background with an intensity comparable to the optical background. This discovery paved the way for large aperture far-infrared and sub-millimeter observations resulting in the discovery of dusty, starbursting galaxies. Their role in galaxy formation and evolution remains an active area of research in modern-day astrophysics. The extreme UV (EUV) background remains mostly unexplored and will be a challenge to measure due to the high Galactic background and absorption of extragalactic photons by the intergalactic medium at these EUV/soft X-ray energies. She also summarizes our understanding of the spatial anisotropies and angular power spectra of intensity fluctuations. She motivates a precise direct measurement of the COB between 0.1 and $5 \mu \mathrm{m}$ using a small aperture telescope observing either from the outer Solar 
System, at distances of $5 \mathrm{AU}$ or more, or out of the ecliptic plane. Other future applications include improving our understanding of the background at $\mathrm{TeV}$ energies and spectral distortions of $\mathrm{CMB}$ and CIB.

Henry et al. (2015) discussed the diffuse cosmic background radiation in the Galaxy Evolution Explorer far-ultraviolet (FUV, 1300-1700). They deduced that the UV diffuse cosmic background radiation originates only partially in the dust-scattered radiation of FUV-emitting stars: the source of a substantial fraction of the FUV background radiation remains a mystery. They also discussed about our limited knowledge of the cosmic diffuse background at ultraviolet wavelengths shortward of Ly $\alpha$ - it could be that a "second component" of the diffuse FUV background persists shortward of the Lyman limit and is the cause of the reionization of the universe.

\subsection{Gamma Ray Bursts}

Gamma-ray burst (GRBs) were discovered in 1967 - thanks to the four VELA spacecrafts, originally designed for verifying whether the Soviet Union abided the 1963 Limited Nuclear Test Ban Treaty - when 16 strong events were detected (Klebesadel, Strong \& Olson, 1973). Since then GRBs have remained a puzzle for the community of high energy astrophysicists. For this reason the problem of GRBs originated thousands articles most of them devoted to their physical interpretation (e.g. the review by Mazets \& Golenetskii, 1988; the review by GSG2004 and the references therein). BATSE/CGRO experiment detected 2704 GRBs from 1991 to 1999. This number increased with new generation satellites (BeppoSAX, RossiXTE, HETE, INTEGRAL, SWIFT, and FERMI). From the BATSE and KONUS isotropic distribution of GRBs and their cosmological origin have been demonstrated. GRBs may be classified into two groups depending on their duration: $\sim 0.2 \mathrm{~s}(25 \%)$, and $\sim 30 \mathrm{~s}(75 \%)$ (e.g. Kouveliotou et al., 1993). The counterparts for all bursts can be observed in all wavelengths (X, UV, opt, IR, radio): the afterglow (e.g. Kann et al., 2010; Perley et al., 2014).

Theoretical description of GRBs is still an open strongly controversial question as discussed elsewhere (e.g. Giovannelli \& Sabau-Graziati, 2008; Giovannelli, 2013). Many review papers have been published about GRBs. Among them we can cite those published in the last decade (Piran, 2004; Meszaros, 2006); Woosley \& Bloom, 2006; Granot, 2007, 2009; Granot \& Ramirez-Ruiz, 2010; Inoue et al., 2013). Recently an interesting review about short GRBs has been published by Berger (2014).

Important implications on the origin of the highest redshift GRBs are coming from the detection of the GRB 080913 at $\mathrm{z}=6.7$ (Greiner et al., 2009), GRB 090423 at $\mathrm{z} \sim 8.2$ (Tanvir et al., 2009), and GRB 090429B at $\mathrm{z}=9.4$ (Cucchiara et al., 2011). This means that really we are approaching to the possibility of detecting GRBs at the end of Dark Era, where the first Pop III stars appeared. Izzo et al. (2010) discussed successfully a theoretical interpretation of the GRB 090423 within their fireshell model. Wang \& Dai (2009) studied the high-redshift star formation rate (SFR) up to $\mathrm{z} \simeq 8.3$ considering the Swift GRBs tracing the star formation history and the cosmic metallicity evolution in different background cosmological models including $\Lambda$ CDM, quintessence, quintessence with a time-varying equation of state and brane-world models. $\Lambda \mathrm{CDM}$ model is the preferred which is however compared with other results.

Although big progress has been obtained in the last few years, GRBs theory needs further investigation in the light of the experimental data coming from old and new satellites, often co- 
ordinated, such as BeppoSAX or BATSE/RXTE or ASM/RXTE or IPN or HETE or INTEGRAL or SWIFT or AGILE or FERMI or MAXI. Indeed, in spite of thousands papers appeared in the literature since the discovery of GRBs, the problem of their energy emission is still elusive: i) what is jet's composition? (kinetic or magnetic?); ii) where is dissipation occurring? (photosphere? deceleration radius?); iii) how is radiation generated? (synchrotron, Inverse Compton, hadronic?) (Zhang, 2013a,b).

For this reason we believe useful to read the very interesting scientific-social remark made by Arnon Dar at the end of the paper discussed by Guido Barbiellini at the Vulcano Workshop 2002 (Barbiellini \& Longo, 2003).

Kumar \& Zhang (2015) in a review paper The Physics of Gamma-Ray Bursts \& Relativistic Jets discussed what we have learned about relativistic collisionless shocks and particle acceleration from GRB afterglow studies, and the current understanding of radiation mechanism during the prompt emission phase. They pointed out how these explosions may be used to study cosmology, e.g. star formation, metal enrichment, reionization history, as well as the formation of first stars and galaxies in the Universe.

The idea that GRBs could be associated to gravitational waves (GWs) emission is now popular. Indeed, short GRBs are believed to be produced by the mergers of either double NSs or NS-BH binaries (Nakar, 2007) and the recent observation of a kilonova associated with GRB130603B (Tanvir et al., 2013; Berger, Fong \& Chornock, 2013) lends support to this hypothesis.

Such compact binary coalescences generate strong GWs in the sensitive frequency band of Earth-based gravitational wave detectors (Blanchet, Iyer \& Joguet, 2002; Blanchet \& Damour, 1989). Aasi et al. (2014) searched for gravitational waves associated with 223 GRBs detected by the InterPlanetary Network (IPN) in 2005-2010 during LIGO's fifth and sixth science runs and Virgo's first, second, and third science runs. No evidence of a gravitational wave signal associated with any of the IPN GRBs in the sample, nor evidence for a population of weak gravitational wave signals associated with the GRBs has been found.

Thanks to the NASA's Swift satellite we assisted to ten years of amazing discoveries in time domain astronomy. Its primary mission is to chase GRBs. The list of major discoveries in GRBs and other transients includes the long-lived X-ray afterglows and flares from GRBs, the first accurate localization of short GRBs, the discovery of GRBs at high redshift $(z>8)$ (Gehrels \& Cannizzo, 2015). And essentially thanks to these discoveries we are now closer to understand the real nature of GRBs.

Indeed, in a recent review, D'Avanzo (2015) discussed the observational properties of short GRBs and showed how the study of these properties can be used as a tool to unveil their elusive progenitors and provide information on the nature of the central engine powering the observed emission. The increasing evidence for compact object binary progenitors makes short GRBs one of the most promising sources of gravitational waves for the forthcoming Advanced LIGO/Virgo experiments.

The recent review by Bernardini (2015) discussed how the newly-born millisecond magnetars can compete with black holes as source of the GRB power, mainly with their rotational energy reservoir. They may be formed both in the core-collapse of massive stars, and in the merger of neutron star or white dwarf binaries, or in the accretion-induced collapse of a white dwarf, being thus a plausible progenitor for long and short GRBs, respectively. She reviewed the major observational 
evidences for the possible presence of a newly-born magnetar as the central engine for both long and short GRBs. She then discussed about the possibility that all GRBs are powered by magnetars, and she proposed a unification scheme that accommodates both magnetars and black holes, connected to the different properties and energetics of GRBs. Since the central engine remains hidden from direct electromagnetic observations, she reviewed the predictions for the GW emission from magnetars hosted from GRBs, and the observational perspectives with advanced interferometers.

Ghirlanda et al. (2015) discussed about the apparent separation of short and long GRBs in the hardness ratio vs duration plot. This separation has been considered as a direct evidence of the difference between these two populations. The origin of this diversity, however, has been only confirmed with larger GRB samples but not fully understood. They concluded that short and long GRBs have similar luminosities and different energetics (i.e. proportional to the ratio of their average durations). Then, it seems that the results are pointing toward the possibility that short and long GRBs could be produced by different progenitors but the emission mechanism responsible for their prompt emission might be similar.

Piron (2016) in his review discussed the updated knowledge of GRBs at very high energies. Their huge luminosities involve the presence of a newborn stellar-mass black hole emitting a relativistic collimated outflow, which accelerates particles and produces non-thermal emissions from the radio domain to the highest energies. He reviewed recent progresses in the understanding of GRB jet physics above $100 \mathrm{MeV}$, based on Fermi observations of bright GRBs, and discussed the physical implications of these observations and their impact on GRB modeling.

Recently Arnon Dar (2017) proposed again to the attention of the international community his Cannonball (CB) model for explaining the physics of GRBs. In the CB model, GRBs and their afterglows are produced by the interaction of bipolar jets of highly relativistic plasmoids (CBs) of ordinary matter with the radiation and matter along their trajectory. Such jetted CBs are presumably ejected in accretion episodes of fall-back material on the newly formed compact stellar object in core-collapse supernovae (SNe) of Type Ic, in merger of compact stellar objects in close binary systems, and in phase transitions in compact stars (Shaviv \& Dar, 1995; Dar, 1997; Dar \& De Rujula, 2000; Dado \& Dar, 2013a). Dado, Dar \& De Rújula (2009) discussed a long series of different SWIFT GRBs, showing that the CB model fits all their broadband light curves. Dado \& Dar (2013b) discussed the jet break in the X-ray afterglow of GRBs that appears to be correlated to other properties of the X-ray afterglow and the prompt gamma ray emission, but the correlations are at odds with those predicted by the conical fireball (FB) model of GRBs (Piran, 1999). On the contrary they are in good agreement, however, with those predicted by the CB model of GRBs.

Finally, Dado \& Dar (2016) discussed on the critical test of gamma-ray burst theories and demonstrated definitively the validity of the CB model against the popular FB model (Piran, 1999).

Figure 32 shows, as example, the fits of light curves of GRB 060729, GRB 061007, GRB 160625B, and GRB 130427A by using the CB model.

In our opinion the problem of the models for explaining the behaviour of GRBs can be considered closed. The CB model is the best in absolute for the description of the physics governing the GRBs. 

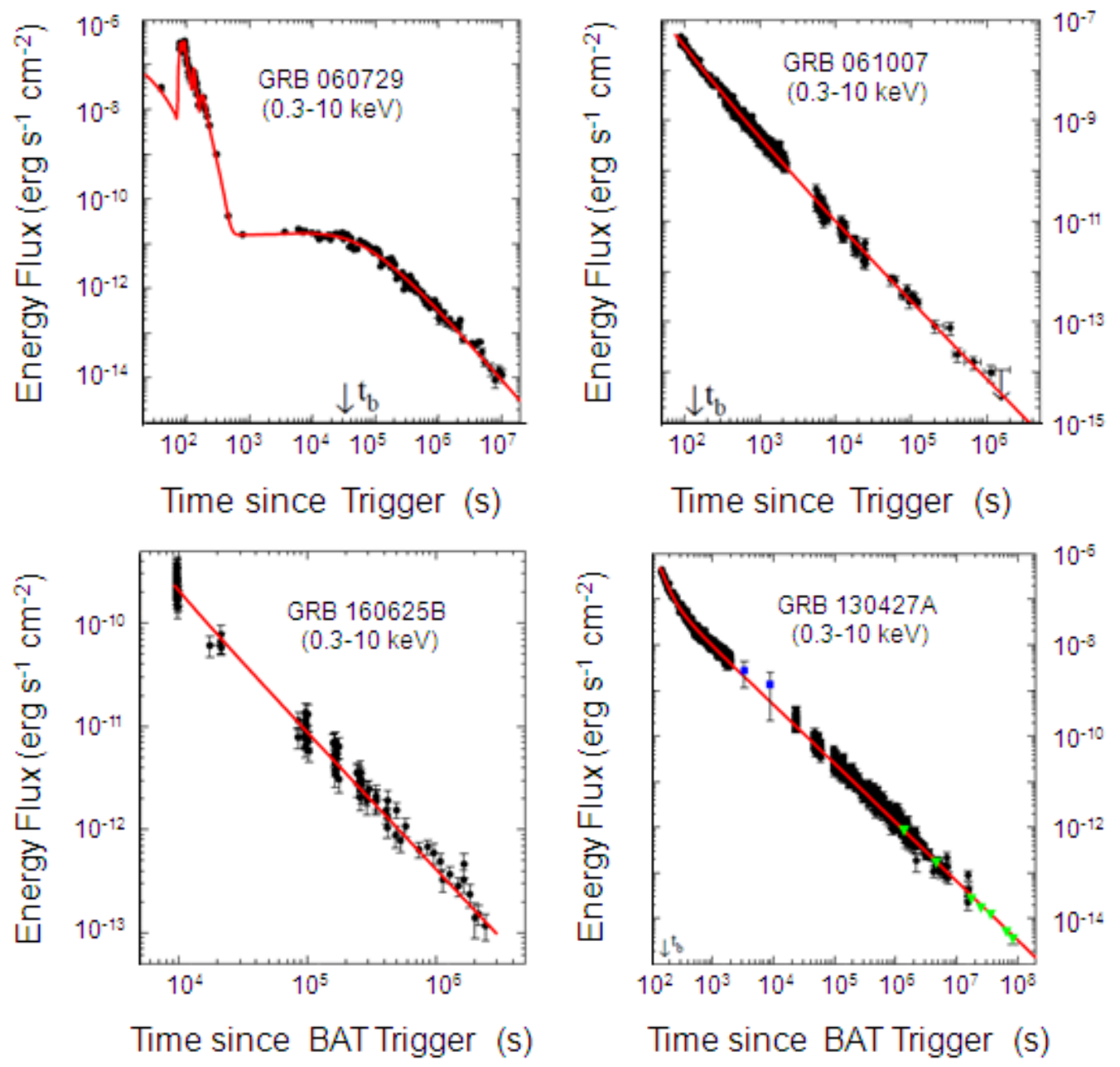

Figure 32: The 0.3-10keV X-ray light-curve measured with the Swift XRT (Evans et al., 2009), and the comparison between Swift observations and their CB-model description (adapted from Dado \& Dar, 2016) for: top left GRB 060729, top right GRB 061007, bottom left GRB 160625B, bottom right GRB 130427A. This latter figure reports data from different experiments: Swift XRT (black circles), XMM Newton and Chandra (green triangles) (De Pasquale et al., 2016), and the two MAXI data points (blue squares) (Maselli et al., 2014) at $\mathrm{t}=3257 \mathrm{~s}$ and $\mathrm{t}=8821 \mathrm{~s}$. The BAT trigger time is marked with $\mathrm{t}_{\mathrm{b}}$.

\section{Accretion Processes}

Accretion is a universal phenomenon that takes place in the vast majority of astrophysical objects. The progress of ground-based and space-borne observational facilities has resulted in the great amount of information on various accreting astrophysical objects, collected within the last decades. The accretion is accompanied by the process of extensive energy release that takes place on the surface of an accreting object and in various gaseous envelopes, accretion disk, jets and other elements of the flow pattern. The results of observations inspired the intensive development of accretion theory, which, in turn, enabled us to study unique properties of accreting objects and physical conditions in the surrounding environment. One of the most interesting outcomes of this intensive study is the fact that accretion processes are, in a sense, self-similar on various spatial 
scales from planetary systems to galaxies.

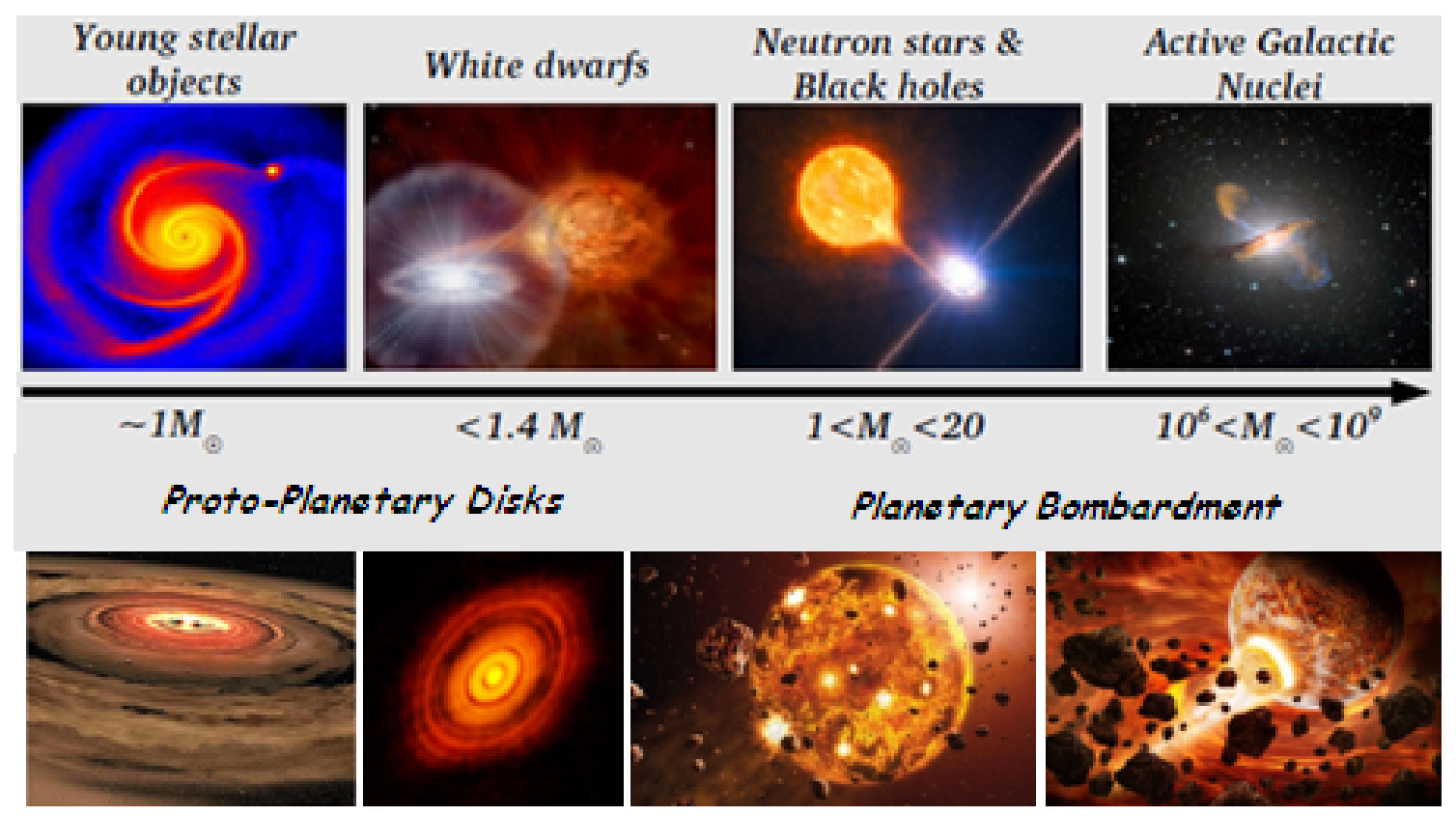

Figure 33: Accretion processes in different cosmic sources (Giovannelli \& Sabau-Graziati, 2016b, after Scaringi, 2015).

This fact gives us new opportunities to investigate objects that, by various reasons, are not available for direct study.

Cataclysmic variable stars are unique natural laboratories where one can conduct the detailed observational study of accretion processes and accretion disks.

Figure 33 shows a sketch of cosmic systems where accretion processes occur (Giovannelli \& Sabau-Graziati, 2016b, after Scaringi, 2015).

An international workshop on Accretion Processes in Cosmic Sources: Young Stellar Objects, Cataclysmic Variables and Related Objects, X-ray Binary Systems, Active Galactic Nuclei have been organized. The proceedings will discuss in details the physics of accretion processes in all the cosmic sources shown in Fig. 33 (Giovannelli \& Sabau-Graziati, 2017).

\section{Habitable Zone in the Milky Way and Exoplanets}

A deep discussion about this fundamental problem has been published by Giovannelli \& Sabau-Graziati (2016a) and references therein. We can briefly summarize in the following.

The most important questions about the possible origin of life in our Universe became a real scientific question in the last couple decades when it appeared a near certainty that other planets must orbit other stars. And yet, it could not be proven, until the early 1990's. Then, radio and optical astronomers detected small changes in stellar emission which revealed the presence of first a few, and now many, planetary systems around other stars. We call these planets "exoplanets" to distinguish them from our own solar system neighbors (http://science.nasa.gov/astrophysics/focusareas/exoplanet-exploration/). 
The research of potential habitable exoplanets has been strongly supported during last two decades. Indeed, this field of astrophysics is now probably the most exciting since the discovery of planets Earth-like could open a serious debate about the possibility of life outside of solar system.

The presence of numerous exoplanets in the vicinity of solar system - within a distance of $\sim 0.8 \mathrm{pc}-$ plays an important role in speculating about the possible number of such exoplanets within the whole habitable zone of our galaxy. Such habitable zone has an internal radius of $\sim 4$ kpc and an external radius of $\sim 11 \mathrm{kpc}$, as shown in Fig. 34 (Giovannelli \& Sabau-Graziati, 2016a after Lineweaver, Fenner \& Gibson, 2004), where the habitable zone in a Milky Way-like galaxy is represented in green. The number of stars contained in this zone is $\approx 10 \%$ of the total number of stars in the Galaxy. Taking into account that the thickness of the disk is $\approx 1 \mathrm{kpc}$, as evaluated by the differential rotation of the Galaxy, the habitable volume is $\sim 330 \mathrm{kpc}^{3}$. Therefore, if in a volume of $\approx 2 \mathrm{pc}^{3}$ there are 808 Earth-like planets detected, in the habitable zone of our Galaxy we could expect $\approx 133 \times 10^{6}$ Earth-like planets. It is evident that the probability of finding numerous habitable planets becomes very high. Next generation instruments ground- and space-based will provide valuable information about this intriguing problem.

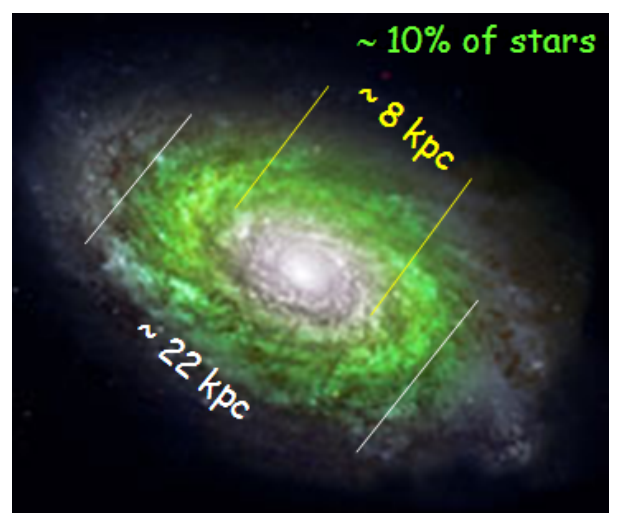

Figure 34: Habitable zone of a Milky Way-like galaxy (Giovannelli \& Sabau-Graziati, 2016a after Lineweaver, Fenner \& Gibson, 2004).

A strong support on the possibility of having numerous habitable planets is coming from the discovery of "usual" presence of water in the universe. We knew that all the water found on Earth, has been transported by small bodies such as comets and asteroids. On the contrary, the work "The ancient heritage of water ice in the solar system" (Cleeves et al., 2014) has carried the knowledge one step further. It is understood that the water now present in Earth's oceans, and is present in other solar system bodies, has remained virtually unchanged with respect to that in the interstellar medium. This means that this water has not changed during the process of planet formation. This allows us to understand that the initial conditions that have favored the emergence of life are not unique, i.e. not dependent on the unique characteristics of our solar system. They can, however, be common in space.

An intriguing question about the probability of finding a number of civilization in the Galaxy arises. It is now evident that Drake's formula (Drake, 1962) must be object of a robust revision.

For years, the search for manifestations of extraterrestrial civilizations has been one of humanity's most ambitious projects. Major efforts are now focused on the interception of mes- 
sages from extraterrestrial civilizations, and the millimeter range is promising for these purposes (Dyson,1960). The Millimetron space observatory is aimed at conducting astronomical observations to probe a broad range of objects in the Universe in the wavelength range $20 \mu \mathrm{m}$ to $20 \mathrm{~mm}$, including the search for extraterrestrial life (Kardashev et al., 2014, and the references therein).

\section{Conclusions}

In this review paper we have discussed several arguments that in our opinion are fundamental for the comprehension of the physics of our Universe. We have emphasized with some examples the use of wisdom in physics. We hope to have given some hints to the readers in order to adopt wisdom during the preparation of their papers. Moreover we invite our colleagues to use the citations of the original papers in order to avoid dangerous chains of references coming usually from the current style, as largely commented in the "Concluding Remarks" of the review paper by Giovannelli (1994) in which he discussed about the methodology of the investigation and on the ethics in science.

It is important the Great example of synergy between Astrophysics and History we discussed for demonstrating that Sedov's formula for determining the age of SNRs can be revisited, thanks to the recalibration of the age of the SNR Vela Jr (Aschenbach, 2016) experimentally supported by an historical document.

We discussed about the small and big space- and ground-based experiments that provide measurements necessary for the advancement of the knowledge of the physics of our Universe. Thanks to these results we discussed the present situation about the problems resolved and those till open, far from the completeness due to our limited knowledge.

A final section about the Habitable Zone in the Milky Way and Exoplanets briefly commented the fundamental problem about the research of life in the Universe. With this new-born field of science we could finally prove that the "Bridge between the Big Bang and Biology" is, not only obviously existing, but really can be traveled over.

Acknowledgments This research has made use of:

- The NASA's Astrophysics Data System;

- the NASA Exoplanet Archive, which is operated by the California Institute of Technology, under contract with the National Aeronautics and Space Administration under the Exoplanet Exploration Program.

\section{References}

[1] Aad, G. (The ATLAS Collaboration): 2012, PhL B 718, 369-390.

[2] Aaij, R. et al.: 2015, arXiv:1507.03414v2 [hep-ex] 25 Jul.

[3] Aasi, J. et al.: 2014, PhRvL 113, 011102.

[4] Abbott, B.P. et al.: 2016a, PRL 116, 061102.

[5] Abbott, B.P. et al.: 2016b, PhRvL 116,1103. 
[6] Abbott, B.P. et al.: 2016c, Living Rev. Relativity 19, 1.

[7] Abbott, B.P. et al.: 2017a, PhRvL 119, Issue 16, id.161101.

[8] Abbott, B.P. et al.: 2017b, ApJL 848, Issue 2, article id. L12, 59 pp.

[9] Abdo, A.A. et al.: 2010, ApJ, 723, 1082.

[10] Ade, P.A.R. et al. (BICEP Collaboration): 2015, PhRvL 114, 1301.

[11] Albert, J. \& Magic Collaboration: 2008, Science, 320, 1752.

[12] Aschenbach, B.: 1998, Nature 396, 141.

[13] Aschenbach, B.: 2016, in Frontier Research in Astrophysics II. Online at https://pos.sissa.it/cgi-bin/reader/conf.cgi?confid=269, id.36.

[14] Barbiellini, G. \& Longo,F.: 2003, in Frontier Objects in Astrophysics and Particle Physics, F. Giovannelli \& G. Mannocchi (Eds.), SIF, Bologna, Italy, 85, 281.

[15] Bartelmann, M., 2008: in Rev. Mod. Astron. (Siegfried Röser Ed.), Vol. 20: Cosmic Matter, p. 92.

[16] Beall, J.H.: 2002, in Multifrequency Behaviour of High Energy Cosmic Sources, F. Giovannelli \& L. Sabau-Graziati (Eds.), Mem. S.A.It. 73, 379.

[17] Beall, J.H.: 2003, in Multifrequency Behaviour of High Energy Cosmic Sources, F. Giovannelli \& L. Sabau-Graziati (Eds.), ChJA\&A Suppl. 3, 373.

[18] Beall, J.H., Guillory, J., Rose, D.V.: 1999, in Multifrequency Behaviour of High Energy Cosmic Sources, F. Giovannelli \& L. Sabau-Graziati (Eds.), Mem. S.A.It. 70, 1235.

[19] Beall, J.H. et al.: 2006, in Multifrequency Behaviour of High Energy Cosmic Sources, F. Giovannelli \& L. Sabau-Graziati (Eds.), ChJA\&A Suppl. 6 Suppl. 1, 283.

[20] Beall, J.H. et al.: 2007, in Frontier Objects in Astrophysics and Particle Physics, F. Giovannelli \& G. Mannocchi (Eds.), SIF, Bologna, Italy 93, 315.

[21] Bednarek, W., Giovannelli, F., Karakuła, S., Tkaczyk, W.: 1990, A\&A 236, 268.

[22] Begelman, M.C., Blandford, R.D. \& Rees, M.J.: 1984, Rev. Mod. Phys. 56, 255.

[23] Bennett, C.L. et al.: 2013, ApJS, 208, 20.

[24] Bennett, C.L. et al.: 2014, ApJ, 794, 135.

[25] Berger, E.: 2014, Annu. Rev. A\&A, 52, 43-105.

[26] Berger, E., Fong, W. \& Chornock, R.: 2013, ApJL 774, L23.

[27] Bernardini, M.G.: 2015, J.HE Astrophys. 7, 64-72.

[28] de Bernardis et al.: 2000, Nature, 404, 955.

[29] de Bernardis et al.: 2014, talk at the Mondello Workshop Frontier Research in Astrophysics.

[30] Bisnovatyi-Kogan, G.S.: 2002, in Black Hole Astrophysics 2002, Proc. of the Sixth APCTP Winter Schoo, Pohang, Korea, pp. 187-206.

[31] Bisnovatyi-Kogan, G.S., Giovannelli, F.: 2017, A\&A 599, id. A55, 7 pp.

[32] Blanchet, L., Damour, T.: 1989, Annales Inst. H. Poincaré Phys. Théor. 50, 377

[33] Blanchet, L., Iyer, B.R., Joguet, B.: 2002, Phys. Rev. D 65, 064005. 
[34] Blandford, R.D., Kochanek, C.S.: 2004, in Dark Matter in the Universe (Second Edition), J. Bahcall et al. (Eds.), World Scientific Publishing Co. Pte. Ltd., ISBN 9789812567185, pp. 103-158.

[35] Bloom, J., Castro-Tirado, A.J., Hanlon, L. \& Kotani, T. (Eds.): 2010, I Workshop on Robotic Autonomous Observatories, Advances in Astronomy, Vol. 2010.

[36] Bucher, M. (on behalf of the PLANCK Collaboration): 2015, Nucl and Part. Phys. Proc. 267-269, 245-253.

[37] Burles, S. et al.: 2001, ApJ, 552, L1.

[38] Buson, S.: 2014, in Frobtier Research in Astrophysics, Franco Giovannelli \& Lola Sabau-Graziati (eds.), http://pos.sissa.it/cgi-bin/reader/conf.cgi?confid=237.

[39] Caballero-García, M.D., Pandey, S.B., Hiriart, D. \& Castro-Tirado, A.J. (Eds.): 2016, IV Workshop on Robotic Autonomous Observatories, RMxAC, Vol. 48.

[40] Cardini, A.: 2015, Interview of Alessandro Cardini, P.I. of LHCb, 14th July 2015.

[41] Castro Cerón, J.M.: 2011, talk at the Frascati Workshop 2011 on "Multifrequency Behaviour of High Energy Cosmic Sources".

[42] Castro-Tirado, A.J.: 2008, in 3rd Symposium of the Astrophysics Group of the Spanish Royal Physical Society (RSEF), A. Ulla \& M. Manteiga (Eds.), Lecture Notes and Essays in Astrophysics 3, 131.

[43] Castro-Tirado, A.J.: 2010a, Adv. Astron. Vol. 2010, Article ID 570489, 8 pages.

[44] Castro-Tirado, A.J.: 2010b, Adv. Astron. Vol. 2010, Article ID 824731, 1 page.

[45] Clarke, D.A. et al.: 2008, Physics in Canada 64(2), 48.

[46] Class for Physics of the Royal Swedish Academy of Sciences: 2011, The Accelerating Universe, The Royal Swedish Academy of Sciences (4th October).

[47] Cleeves L. Ilsedore et al.: 2014, Science, 345, 1590.

[48] Cooray, A.: 2016, R. Soc. open sci. 3: 150555. http://dx.doi.org/10.1098/rsos.150555.

[49] Coppi, P.S. \& Aharonian, F.A.: 1997, ApJ, 487, L9.

[50] Costamante, L.: 2012, in Multifrequency Behaviour of High Energy Cosmic Sources, F. Giovannelli \& L. Sabau-Graziati (Eds.), Mem. SAIt., 83, 138.

[51] Crites, A.T. et al.: 2015, ApJ 805, 36.

[52] Cucchiara, A., Levan, A.J., Fox, D.B., Tanvir, N.R., Ukwatta, T.N. et al.: 2011, ApJ 736, Issue 1, article id. 7, $12 \mathrm{pp}$.

[53] Dai, X., Kochanek, C.S.: 2005, ApJ 625, 633.

[54] Dado, S., Dar, A., De Rújula, A.: 2009, ApJ 696, Issue 1, 994-1020.

[55] Dado, S., Dar, A.: 2013a, ApJ 775, Issue 1, article id. 16, 7 pp.

[56] Dado, S., Dar, A.: 2013b, A\&A 558, id.A115, 7 pp.

[57] Dado, S., Dar, A.: 2016, PhRvD 94, Issue 6, id.063007.

[58] Damon, E. et al.: 2004, Measurements of the Hubble Constant, D.B. Campbell \& J. Deneva (eds.), Proc. of the Fall 2004 Astronomy 233 Symposium, Cornell University Astronomy Department and the College of Arts and Sciences under the John S. Knight Institute Sophomore Seminar Program, pp. $1-61$. 
[59] Dar, A.: 1997, in Very High Energy Phenomena in the Universe Morion Workshop. ISBN 2-86332-217-6 Editions Frontieres, Edited by Y. Giraud-Heraud and J. Tran Thanh Van, p.69. (arXiv:astro-ph/9704187v2).

[60] Dar, A.: 2017, talk at the Frascati Workshop 2017 Multifrequency Behaviour of High Energy Cosmic Sources - XIII.

[61] Dar, A., De Rújula, A.: 2000, arXiv:astro-ph/0008474.

[62] D’Avanzo, P.: 2015, J. HE Astrophys. 7, 73-80.

[63] De Angelis, A. et al.: 2017, Exp. Astron. 44, Issue 1, 25-82.

[64] De Pasquale, M., Page, M.J., Kann, D.A., Oates, S.R., Schulze, S. et al.: 2016, MNRAS 462, Issue 1, 1111-1122.

[65] Domínguez, A. et al.: 2011, MNRAS, 410, 2556.

[66] Dopita, M.A., Krauss, L.M., Sutherland, R.S., Kobayashi, C., Lineweaver, C.H.: 2011, Astrophys. Space Sci., 335, 345.

[67] Drake, F.D.: 1962, Intelligent Life in Space, New York: Macmillan, 128 pp.

[68] Durrer, R.: 2015, Classical and Quantum Gravity, 32, Issue 12, article id. 124007.

[69] Dyson, F.: 1960, Science, 131, 1667.

[70] Erickson, J.K., Gratton, S., Steinhardt, P.J., Turok, N.: 2007, PhRvD 75, Issue 12, id. 123507.

[71] Evans, P.A. et al.: 2009, MNRAS 397, 1177 [arXiv:0812.3662].

[72] Fan, X. et al.: 2003, AJ 125, 1649.

[73] Flauger, R., Hill, J.C., Spergel, D.N.: 2014, JCAP 1408, 039. (arXiv:1405.7351)).

[74] Freedman, W.L., Madore, B.F.: 2010, Annu. Rev. A\&A, 48, 673-710.

[75] Gehrels, N., Cannizzo, J.K.: 2015, J. HE Astrophys. 7, 2-11.

[76] Gell-Mann, M.: 1964, PhL, 8, 214.

[77] Ghirlanda, G., Bernardini, M.G., Calderone, G., D’Avanzo, P.: 2015, J. HE Astrophys. 7, 81-89-

[78] Giallongo, E. et al.: 1994, ApJ, 425, L1.

[79] Gianotti, F.: 2012, 4th July, talk at CERN.

[80] Gilmozzi, Roberto: 2013, talk at "The Golden Age of Cataclysmic Variables and Related Objects - II" workshop, Palermo 9-14 September.

[81] Gilmozzi, Roberto, Spyromilio, Jason: 2007, The Messenger 127, 11-19.

[82] Giovannelli, F.: 1994, SSRv 69, Issue 1-2, 1-138.

[83] Giovannelli, F. (ed.): 2001, The Bridge between the Big Bang and Biology (Stars, Planetary Systems, Atmospheres, Volcanoes: Their Link to Life), President Bureau of the CNR, Roma, Italy, pp. 1-440.

[84] Giovannelli, F.: 2010, in Multifrequency Behaviour of High Energy Cosmic Sources, F. Giovannelli \& L. Sabau-Graziati (Eds.), Mem. SAIt 81, 510.

[85] Giovannelli, F.: 2013, Acta Polytechnica 53(Suppl.), 483.

[86] Giovannelli, F., Polcaro, V.F.: MNRAS 222, 619-627. 
[87] Giovannelli, F., Errico, L., Vittone, A.A., Rossi, C.: 1991, A\&A Sup. Ser. 87, no. 1, 89-95.

[88] Giovannelli, F., Sabau Graziati, L., La Padula, C., Errico, L., Frutti, M. et al.: 1993, A\&AS 97, 395.

[89] Giovannelli, F., Vittone, A.A., Rossi, C., Errico, L., Bisnovatyi-Kogan, G.S., Kurt, V.G., Lamzin, S.A., Larionov, M., Sheffer, E.K., Sidorenkov, V.N.: 1995, A\&A Supp. Ser. 114, 341-361.

[90] Giovannelli, F., Sabau-Graziati, L., La Padula, C.D., Isern, J., Bravo, E., Garcia-Berro, E., Gómez-Gomar, J., Guerrero, J., Hernans, M., José, J.: 1999a, Mem. SAIt. 70, 1317-1346.

[91] Giovannelli, F., La Padula, C.D., Sabau-Graziati, L., Talavera, A., Angulo, M., Isern, J. et al.: 1999b, in Frontier Objects in Astrophysics and Particle Physics, F. Giovannelli \& G. Mannocchi (Eds.), Italian Physical Society 65, 623-644.

[92] Giovannelli, F., Sabau-Graziati, L., Isern, J., La Padula, C.D., Angulo, M. et al.: 2001, in Frontier Objects in Astrophysics and Particle Physics, F. Giovannelli \& G. Mannocchi (Eds.), Italian Physical Society 73, 565-579.

[93] Giovannelli, F., Sabau-Graziati, L., Isern, J., La Padula, C.D., Angúlo, M., Badenes, C., Bausells, J., Bravo, E., Cabestany, J., Fernández-Valbuena, M., Frutti, M., Ganzo, F., García-Berro, E., García-Senz, D., Gómez-Gomar, J., Gutiérrez, J., José, J., Hernanz, M., Herrera, E., del Llano, R., Madrenas, J., Réina, M., Talavera, A.: 2002a, SIXE (Spanish Italian X-ray Experiment) - Feasibility Study Report. (PNIE-CICYT Report (ESP97-1784-E grant), IASF (Istituto di Astrofisica Spaziale e Fisica Cosmica, Roma), Technical Report No. 4, December 2002.

[94] Giovannelli, F., Sabau-Graziati, L., Isern, J., La Padula, C.D., Angulo, M. et al.: 2002b, Mem. SAIt 73, 402-417.

[95] Giovannelli, F., Sabau-Graziati, L.: 2004, The Impact of Space Experiments on our Knowledge of the Physics of the Universe, Reprinted from Space Science Reviews, Vol. 112, Nos. 1-4, pp. 1-443, Kluwer Academic Publishers, Dordrecht (GSG2004).

[96] Giovannelli, F. \& Sabau-Graziati, L.: 2008, Chinese J. A\&A Suppl., 8, 1.

[97] Giovannelli, F., Sabau-Graziati, L.: 2011, Acta Polytechnica Vol. 51, No. 2., p. 21.

[98] Giovannelli, F., Bisnovatyi-Kogan, G.S., Klepnev, A.S.: 2013, A\&A 560, id.A1, 11 pp.

[99] Giovannelli, F., Sabau-Graziati, L.: 2014a, in Frontier Research in Astrophysics, Franco Giovannelli \& Lola Sabau-Graziati (eds.), http://pos.sissa.it/cgi-bin/reader/conf.cgi?confid=237

[100] Giovannelli, F., Bisnovatyi-Kogan, G.S., Bruni, I., Corfini, G., Martinelli, F., Rossi, C.: 2015, AcA $65,107$.

[101] Giovannelli, F., Sabau-Graziati, L.: 2016a, in Frontier Research in Astrophysics II, Online at https://pos.sissa.it/cgi-bin/reader/conf.cgi?confid=269, id. 1.

[102] Giovannelli, F., Sabau-Graziati, L.: 2016b, talk at the Saint Petersburg Workshop on Accretion Processes in Cosmic Sources: Young Stellar Objects, Cataclysmic Variables and Related Objects, $X$-ray Binary Systems, Active Galactic Nuclei.

[103] Giovannelli, F., Sabau-Graziati, L. (Eds.): 2017, Accretion Processes in Cosmic Sources: Young Stellar Objects, Cataclysmic Variables and Related Objects, X-ray Binary Systems, Active Galactic Nuclei, PoS-SISSA (in press).

[104] Gnedin, N.Y.: 2000, ApJ, 535, 530-554.

[105] de Gouveia dal Pino, E.M.: 2005, Adv. in Space Res. 35, 908. 
[106] de Gouveia Dal Pino, E.M., Piovezan, P.P. \& Kadowaki, L.H.S.: 2010, A\&A 518, A5.

[107] Granot, J.: 2007, Rev.Mex, A\&A (Serie de Conferencias), 27, 140-165.

[108] Granot, J.: 2009, arXiv:0905.2206v1 [astro-ph.HE] 13 May 2009.

[109] Granot, J. \& Ramirez-Ruiz, E.: 2010, arXiv:1012.5101v1 [astro-ph.HE] 22 Dec 2010.

[110] Greiner, J., Krühler, T., Fynbo, J.P.U., Rossi, A., Schwarz, R. et al.: 2009, ApJ 693, 1610-1620.

[111] Guziy, S., Pandey, S.B., Tello, J.C. \& Castro-Tirado, A.J. (Eds.): 2012, II Workshop on Robotic Autonomous Observatories, Astron. Soc. of India Conf. Ser., Vol. 7.

[112] Hamuy, M. et al.: 2012, Mem. S.A.It. Vol. 83, 388.

[113] Haridasu, B.S., Lukovic, V.V., D’Agostino, R., Vittorio, N.: 2017, A\&A 600, id. L1, 5 pp.

[114] Hartwig, T., Volonteri, M., Bromm, V., Klessen, R.S., Barausse, E. et al.: 2016, MNRAS Lett. 460, Issue 1, L74-L78.

[115] Hasinger, G., Miyaji, T., Schmidt, J.H.M.M.: 2000, MPE-Report 1999, Vol. 273, 83.

[116] Henry, R.C.: 1999, ApJL, 516, L49.

[117] Henry, R.C.: 2002, in Multifrequency Behaviour of High Energy Cosmic Sources, Franco Giovannelli \& Lola Sabau-Graziati (eds.), Mem. SAIt., 73 N. 1, 67.

[118] Henry, R.C., Murthy, J., Overduin, J., Tyler, J.: 2015, ApJ, 798, Issue 1, article id. 14, 25 pp.

[119] Huchra, J.: 2007, https://www.cfa.harvard.edu/ dfabricant/huchra/hubble.plot.dat

[120] Huchra, J.: 2008, home page - https://www.cfa.harvard.edu/ dfabricant/huchra/

[121] Hudec, R. et al,: 2017, talk at the Frascati Workshop 2017 on Multifrequency Behaviour of High Energy Cosmic Sources - XII, Mondello, Palermo, Italy, 12-17 June.

[122] Ijjas, A., Steinhardt, P.J., Loeb, A.: 2013, PhLB 723, 261.

[123] Ijjas, A.: 2016, arXiv161002752.

[124] Incandela, J.: 2012, UCSB/CERN, Talk on July 4, 2012.

[125] Inoue, S., Granot, J., O’Brien, P.T., Asano, K., Bouvier, A. et al.: 2013, APh 43, 252-275.

[126] Isern, J., Giovannelli, F. (Principal Investigators), Angúlo, M., Bausells, J., Bravo, E., Cabestany, J., García-Berro, E., Gómez-Gomar, J., Hernanz, M., La Padula, C.D., Sabau-Graziati, L., Talavera, A. (Co-Investigators): 1999a, SIXE (Spanish Italian X-ray Experiment) - Feasibility Study Report, PNIE-CICYT Report (ESP97-1784-E grant).

[127] Isern, J., Bravo, E., Gómez-Gomar, J., Hernanz, M., García-Berro, E., Giovannelli, F., La Padula, C.D., Sabau, L. et al.: 1999b, AIPC 499, 110.

[128] Izzo, L. et al.: 2010, J. Korean Phys. Soc. 57, Issue 31, 551.

[129] Jakobs, K., Seez, C.: 2015, Scholarpedia 10(9):32413.

[130] Jiang, L., Egami, E., Kashikawa, N., Walth, G., Matsuda, Y. et al.: 2011, ApJ, 743, 65.

[131] Kann, D.A. et al.: 2010, ApJ, 720, 1513.

[132] Kaplinghat, M. et al.: 2003, ApJ, 583, 24.

[133] Kardashev, N.S. et al.: 2014, Phys. Uspekhi, 57 (12), 1199. 
[134] Kashikawa, N., 2007, in At the Edge of the Universe: Latest Results from the Deepest Astronomical Surveys, Alfonso, J., Ferguson, H.C., Mobasher, B. \& Norris, R. (Eds.), ASP Conf. Ser., 380, 11

[135] Kashikawa, N., Shimasaku, K., Malkan, M.A., Doi, M., Matsuda, Y. et al., 2006, 648, 7

[136] Keating, Brian G. et al.: 2003, SPIE 4843, 284.

[137] Klebesadel, R.W., Strong, I.B., Olson, R.A.: 1973, ApJL 182, L85.

[138] Knox, L.: 2003, New Astron. Rev., 47, 883.

[139] Kochanek, C.S.: 2003, ASPC 291, 245.

[140] Kogut, A. et al.: 2003, ApJS, 148, 161.

[141] Komatsu, E. et al.: 2011, ApJS, 192, 18.

[142] Kouveliotou, C. et al.: 1993, ApJ 413, L101.

[143] Kumar, P., Zhang, B.: 2015, PhR 561, 1-109.

[144] Lamzin, S.A., Bisnovatyi-Kogan, G.S., Errico, L., Giovannelli, F., Katysheva, N.A., Rossi, C., Vittone, A.: 1996, A\&A 306, 877-891.

[145] Lasota, J.-P.: 2001, New Astron. Rev. 45, 449.

[146] LIGO Scientific Collaboration and Virgo Collaboration: 2017, ApJL 850, L40 (18pp).

[147] Lineweaver, C.H., Fenner, Y., Gibson, B.K.: 2004, Nature, 303, 59.

[148] Lipunov, V. et al.: 2010, Advances in Astronomy, 2010, article id. 349171.

[149] Loeb, A. \& Barkana, R.: 2001, Annu. Rev. A\&A, 39, 19.

[150] Marscher, A.P.: 2005, Mem. S.A.It. 76, 13.

[151] Maselli, A., Melandri, A., Nava, L., Mundell, C.G., Kawai, N. et al.: 2014, Science 343, Issue 6166, 48-51.

[152] Matsuoka, Y., Onoue, M., Kashikawa, N., Iwasawa, K., Strauss, M.A. et al.: 2016, ApJ, 828, 26.

[153] Mazets, E.P., Golenetskii, S.V.: 1988, Sov. Sci. Rev. E. Astrophys. Space Phys. 6, 283.

[154] Meszaros, P.: 2006, Rep. Prog. Phys. 69, 2259.

[155] Meylan, G., Jetzer, P., North, P., Schneider, P., Kochanek, C.S., Wambsganss, J.: 2006, Gravitational lensing: strong, weak and micro, G. Meylan, P. Jetzer \& P. North (Eds.), Berlin: Springer, ISBN 3-540-30309-X, ISBN 978-3-540-30309-1, XIII + 552 pp. (Kochanek, C.S.: 2004, arXiv:astro-ph/0407232).

[156] Mineo, S., Gilfanov, M., Lehmer, B.D., Morrison, G.E., Sunyaev, R.: 2014, MNRAS 437, 1698-1707.

[157] Nakar, E.: 2007, Phys. Rep. 442, 166.

[158] Nielsen, J.T., Guffanti, A., Sarkar, S. 2016, NatSR 6, id. 35596.

[159] Ogburn, R.W., IV et al.: 2010, SPIE 7741E..1GO.

[160] Ono, Y., Mouchi, M., Mobasher, B., Dickinson, M., Penner, K. et al.: 2012, ApJ, 744, 83.

[161] Orosz, J.A., Remillard, R.A., Bailyn, C.D., McClintock, J.E.: 1997, ApJL 478, L83.

[162] Ouchi, M., Mobasher, B., Shimasaku, K., Ferguson, H.C., Fall, M. et al.: 2009a, ApJ, 706, 1136. 
[163] Ouchi, M., Ono, Y., Egami, E., Saito, T., Oguri, M. et al.: 2009b, ApJ, 696, 1164.

[164] Ouchi, M., Shimasaku, K., Furusawa, H., Saito, T., Yoshida, M. et al.: 2010, ApJ, 723, 869.

[165] Panagia, N. et al.: 2005, ApJ, 633, L1.

[166] Perley, D.A. et al.: 2014, ApJ, 781, 37.

[167] Perlmutter, S., Aldering, G., Goldhaber, G., Knop, R.A., Nugent, P. et al.: 1999, ApJ 517, Issue 2, 565-586.

[168] Pignata, G. et al.: 2009, AIPC, 1111, 551.

[169] Piran, T.: 1999, Phys. Rep. 314, Issue 6, 575-667.

[170] Piran, T.: 2004, Rev. Mod. Phys., 76, 1143.

[171] Piron, F.: 2016, C. R. Physique 17, 617-631.

[172] Planck Collaboration: 2014, A\&A 571, id. A11, 37 pp.

[173] Ramaty, R., Lingenfelter, R.E.: 1982, Annu. Rev. Nucl. Part. Sci. Vol. 32, 235-269.

[174] Rees, M.J.: 1988, in Origins, A.C. Fabian (ed.), Cambridge University Press, 1

[175] Reig, P., Fabregat, J., Coe, M.J.: 1997, A\&A 322, 193.

[176] Ressel, M.T., Turner, M.S.: 1990, Comm. Astrophys. 14, 323.

[177] Riess, A.G., Filippenko, A.V., Challis, P., Clocchiatti, A., Diercks, A. et al.: 1998, AJ 116, Issue 3, 1009-1038.

[178] Riess, A.G. et al.: 2004, ApJ, 607, 665.

[179] Riess, A.G. et al.: 2011, ApJ, 730, 119.

[180] Scaringi, S.: 2015, talk at the Palermo Workshop on The Golden Age of CVs and Related Objects Ü III.

[181] Schuecker, P.: 2005, In Rev. Mod. Astron. (Siegfried Röser Ed.), Vol. 18, 76-105.

[182] Schuecker, P. et al.: 2003, A\&A, 402, 53.

[183] Shaviv, N.J., Dar, A.: 1995, MNRAS 277, Issue 1, 287-296.

[184] Smoot, G.F.: 2007, Rev. Mod. Phys., 79, 1349.

[185] Smoot, G. F., et al.: 1992, ApJL, 396, L1-L5.

[186] Shahbaz, T., Bandyopadhyay, R.M., Charles, P.A., Wagner, R.M., Muhli, P., et al.: 1998, MNRAS $300,1035$.

[187] Shakura, N.I., Sunyaev, R.A.: 1973, A\&A 24, 337.

[188] Shandarin, Sergei, Habib, Salman, Heitmann, Katrin: 2010, PhRv D, 81, Issue 10, id. 103006.

[189] Shimokawabe, T. et al.: 2009, AIPC 1133, 79.

[190] Smak, J.: 1984, PASP 96, 5.

[191] Spergel, D.N. et al.: 2003, ApJS, 148, 175.

[192] Spillantini, P.: 2008, talk at the Frontier Objects in Astrophysics and Particle Physics Workshop, Vulcano (Italy) 26-31 May. 
[193] Spillantini, P.: 2009, in Frontier Objects in Astrophysics and Particle Physics, F. Giovannelli \& G. Mannocchi (Eds.), Conf. Proc. SIF 98, 39-54.

[194] Srianand, R., Petitjean, P. \& Ledoux, C.: 2000, Nature, 408, 931.

[195] Steinhardt, P.J., Turok, N., Starkman, G.D.: PhT 61, Issue 1, 59.

[196] Sunyaev, R.A. \& Zeldovich, Ya.B.: 1980, MNRAS, 190, 413.

[197] Tanvir, N.R., Fox, D.B., Levan, A.J., Berger, E., Wiersema, K. et al.: 2009, Nature, 461, 1254-1257.

[198] Tanvir, N.R., Levan, A.J., Fruchter, A.S., Hjorth, J., Hounsell, R.A. et al.: 2013, Nature, 500, 547-549.

[199] Tello, J.C., Riva, A., Hiriart, D. \& Castro-Tirado, A.J. (Eds.): 2014, III Workshop on Robotic Autonomous Observatories, RMxAC, Vol. 45.

[200] Teymourian, A.: 2004, in Measurements of the Hubble Constant, D.B. Campbell \& J. Deneva (eds.), Proc. of the Fall 2004 Astronomy 233 Symposium, Cornell University Astronomy Department and the College of Arts and Sciences under the John S. Knight Institute Sophomore, p. 58.

[201] Tonry, J.L. et al.: 2003, ApJ, 594, 1-24.

[202] Topchiev, N.P. et al.: 2016a, J. Ph. Conf. Ser. 675, 032009.

[203] Topchiev, N.P. et al.: 2016b, J. Ph. Conf. Ser. 675, 032010.

[204] Topchiev, N.P. et al.: 2017, J. Ph. Conf. Ser. 798, 012011.

[205] Toshikawa, J., Kashikawa, N., Ota, K., Morokuma, T., Shibuya, T. et al.: 2012, ApJ, 750, 137.

[206] Turner, M.S., Riess, A.G.: 2002, ApJ 569, 18-22.

[207] Tyson, J.A.: 2000, Encyclopedia of Astronomy and Astrophysics, Edited by Paul Murdin, article 2144.

[208] Tyson, J.A., Kochanski, G.P., Dell' Antonio, I.P.: 1998, ApJL 498, Issue 2, L107-L110.

[209] Villada, M., Rossi, C., Polcaro, V.F., Giovannelli, F: 1999, A\&A 344, 211.

[210] Wang, F.Y., Dai, Z.G.: 2009, MNRAS 400, Issue 1, L10-L14.

[211] Wheatley, P.J., Mauche, C.W., Mattei, J.A.: 2003, MNRAS 345, 49.

[212] Williams, L.L.R., Schechter, P.L.: 1997, arXiv:astro-ph/9709059, and Astron.\& Geophys. 38, Issue 5,10 .

[213] Winn, J.N., Rusin, D., Kochanek, C.S.: 2004, Nature 427, Issue 6975, 613-615.

[214] Winstein, B.: 2007, Int. J. Mod. Phy. D 16, Issue 12b, 2563.

[215] Winstein, B.: 2009, in From Quantum to Cosmos: Fundamental Physics Research in Space, Turyshev, Slava G. (Ed.), Published by World Scientific Publishing Co. Pte. Ltd., ISBN 9789814261210, pp. 697-705.

[216] Wittman, D.M., Tyson, J.A., Kirkman, D., Dell'Antonio, I., Bernstein, G.: 2000, Nature 405, Issue 6783, 143-148.

[217] Woosley, S.E., Bloom, J.S.: 2006, Annu. Rev. A\&A, 44, 507.

[218] Wu, Xiangping: 2008, Talk at the Summer School on Cosmic Reionization at the KIAA- PKU, Beijing, China, July 1-11. 
[219] Zaldarriaga, M.: 1997, Phys. Rev. D, 55, 1822.

[220] Zaroubi, S.: 2013, in The First Galaxies, ASSL, 396, 45-104.

[221] de Zeeuw, Tim, Tamai, Roberto, Liske, Jochen: 2014, The Messenger 158, 1-6.

[222] Zhang, B.: 2013a talk at the "Multi-Messenger Transient Workshop, KIAA, China.

[223] Zhang, B.: 2013b, in Gamma-ray Bursts: 15 Years of GRB Afterglows, A.J. Castro-Tirado, J.Gorosabel and I.H. Park (Eds.), EAS Publications Series 61, 285-293. 